\title{
WEIGHTED ENERGY-DISSIPATION PRINCIPLE FOR GRADIENT FLOWS IN METRIC SPACES
}

\author{
RICCARDA ROSSI, GIUSEPPE SAVARÉ, ANTONIO SEGATTI, AND ULISSE STEFANELLI
}

\begin{abstract}
This paper develops the so-called Weighted Energy-Dissipation (WED) variational approach for the analysis of gradient flows in metric spaces. This focuses on the minimization of the parameter-dependent global-in-time functional of trajectories

$$
\mathcal{I}_{\varepsilon}[u]=\int_{0}^{\infty} \mathrm{e}^{-t / \varepsilon}\left(\frac{1}{2}\left|u^{\prime}\right|^{2}(t)+\frac{1}{\varepsilon} \phi(u(t))\right) \mathrm{d} t,
$$

featuring the weighted sum of energetic and dissipative terms. As the parameter $\varepsilon$ is sent to 0 , the minimizers $u_{\varepsilon}$ of such functionals converge, up to subsequences, to curves of maximal slope driven by the functional $\phi$. This delivers a new and general variational approximation procedure, hence a new existence proof, for metric gradient flows. In addition, it provides a novel perspective towards relaxation.
\end{abstract}

\section{INTRODUCTION}

The study of gradient flows has attracted remarkable attention since the late '60s, starting from the pioneering works [Kōm67, CP69, CL71, Bré71, Bré73], where existence and approximation results have been established, in the Hilbert framework, for convex or $\lambda$-convex driving energy functionals. The extension of the existence theory, still in Hilbert spaces, to highly nonconvex (as dominated concave perturbations of convex) energies, see [RS06], hinges on the variational approach to gradient flow evolution which in fact dates back to the seminal work by E. DE GIORGI and coworkers [DGMT80a, MST89, DG93] on the theory of Minimizing Movements and Curves of Maximal Slope. This approach is indeed at the heart of the theory of gradient flows in metric spaces [Amb95, AGS08]. In turn, this theory provides the basis for the interpretation à la Отто [JKO98, Ott01] of a wide class of evolution equations and systems as gradient flows in the Wasserstein spaces of probability measures, in close connection with the theory of Optimal Transport [Vil09].

The focus of this paper is on gradient flows in metric spaces, but the motivation stems from the Hilbert theory. By detailing the results announced in [RSSS11, Seg13], we extend to the metric framework the analysis carried out, in the context of a Hilbert space $H$, in [MS11]. Therein, the (Cauchy problem for the) gradient flow

$$
\left\{\begin{array}{l}
u^{\prime}(t)+\partial \phi(u(t)) \ni 0 \quad \text { in } H, \quad \text { for a.a. } t \in(0, T) \\
u(0)=x
\end{array}\right.
$$

driven by a (proper) lower semicontinuous and $\lambda$-convex energy functional $\phi: H \rightarrow(-\infty, \infty]$, with $\partial \phi: H \rightrightarrows H$ the subdifferential of $\phi$ in the sense of convex analysis, was studied from a novel perspective. Namely, the authors considered the functional of trajectories $u \in H^{1}(0, T ; H) \rightarrow$ $(-\infty, \infty]$ defined by

$$
\mathcal{I}_{\varepsilon, T}[u]:=\int_{0}^{T} \mathrm{e}^{-t / \varepsilon}\left(\frac{1}{2}\left|u^{\prime}(t)\right|^{2}+\frac{1}{\varepsilon} \phi(u(t))\right) \mathrm{d} t
$$

Date: Januray 5, 2018.

Key words and phrases. Gradient flow, metric space, curve of maximal slope, Weighted Energy-Dissipation functionals, variational principle, dynamic programming, Hamilton-Jacobi equation.

G.S. has been partially supported by MIUR via PRIN 2015 project "Calculus of Variations", by Cariplo foundation and Regione Lombardia via project Variational evolution problems and optimal transport, and by IMATI-CNR. R. R. and A. S. acknowledge support from GNAMPA (Gruppo Nazionale per l'Analisi Matematica, la Probabilità e le loro Applicazioni) of INdAM (Istituto Nazionale di Alta Matematica). U.S. acknowledges the support by the Vienna Science and Technology Fund (WWTF) through Project MA14-009 and by the Austrian Science Fund (FWF) projects F 65, I 2375, and P 27052. 
which features both the energy and the (quadratic) dissipation terms, with an exponential weight, and is thus referred to as WED (Weighted Energy-Dissipation) functional. In [MS11] it was shown that: (i) for every $\varepsilon>0$ there exists a unique curve $u_{\varepsilon}$ minimizing the latter functional over all trajectories starting from a given datum $x$; (ii) the minimizers $u_{\varepsilon}$ converge as $\varepsilon \downarrow 0$ to the unique solution of (1.1).

This convergence result resides on the observation that minimizers $u_{\varepsilon}$ of $\mathcal{I}_{\varepsilon, T}$ solve the EulerLagrange equation

$$
-\varepsilon u_{\varepsilon}^{\prime \prime}(t)+u_{\varepsilon}^{\prime}(t)+\partial \phi\left(u_{\varepsilon}(t)\right) \ni 0 \quad \text { in } H, \quad \text { for a.a. } t \in(0, T) .
$$

This is nothing but an elliptic-in-time regularization of the gradient flow problem (1.1), which is formally recovered by taking the limit $\varepsilon \rightarrow 0$. Besides providing an alternative method for proving existence results for (1.1), constructing solutions as (limits of) minimizers of functionals on entire trajectories paves the way to relaxation. Indeed, the convergence result in [MS11] is extended to sequences of approximate minimizers, which shows that, in the case $\phi$ is neither $\lambda$-convex nor lower semicontinuous, one may consider the relaxation of $\mathcal{I}_{\varepsilon}$ (provided it is itself the WED functional for a suitable lower semicontinuous and $\lambda$-convex energy).

The idea of regularizing evolution problems by singular elliptic perturbations in time has been pioneered by Lions [Lio63, Lio63b, Lio65] and used by KoHn \& NirenBerg [KN65] and OleinIK [Ole64] as a tool to inspect regularity. An account of these techniques in the linear case can be found in the book by Lions \& MAGENES [LM72].

The variational view to such elliptic regularization via WED functionals has to be traced back at least to ILMANEN [Ilm94], whose proof of existence and partial regularity of the Brakke meancurvature flow of varifolds is based on this variational technique. An occurrence of WED functionals in the proof of existence of periodic solutions to gradient flows is due to HiRANo [Hir94], and this variational approach is mentioned in the classical textbook by EvANs [Eva98, Problem 3, p. 487].

The WED approach to gradient flows has been initiated by CONTI \& ORTIZ [CO08], who presented two examples of relaxation related with micro-structure evolution. As mentioned, the corresponding theoretical analysis in [MS11] and one can find an early application to the case of mean-curvature evolution of Cartesian surfaces is in [SS11]. An extension of the abstract theory to nonpotential perturbations of gradient flows is by MELCHIONNA [Mel16], while $\lambda$-convex energies are treated in [AS16]. Bögelein, DuZaAR, \& Marcellini [BDM14, BDMS17] recently used this variational approach to prove the existence of variational solutions to the equation

$$
u_{t}-\nabla \cdot f(x, u, \nabla u)+\partial_{u} f(x, u, \nabla u)=0
$$

where the field $f$ is convex in $(u, \nabla u)$, see also Sec. 8.1.

Doubly nonlinear evolution equations have also been tackled by WED methods. In the case of rate-independent processes, the abstract theory is developed by MiELKE \& ORTIZ [MO08], see also the subsequent [MS11], and an application to crack-front propagation in brittle materials has been presented by Larsen, Ortiz, \& Richardson [LOR09]. The rate-dependent case is in turn addressed by the series of contributions [AM17, AS10, AS11, AS14, AMS17]. The reader is additionally referred to LIERo \& MELCHIONNA [LM17] for a stability result via $\Gamma$-convergence and to MeLChIOnNa [Mel17] for an application to the study of qualitative properties of solutions.

The WED variational approach can be applied to certain classes of hyperbolic problems as well. Indeed, De GIORGI conjectured this possibility in the setting of semilinear waves [DG96]. Such conjecture has been positively checked in [Ste11] for the finite-time case and by SERRA \& TILLI [ST12] for the original, infinite-time case. Extensions to mixed hyperbolic-parabolic semilinear equations [LS13a], to different classes of nonlinear energies [LS13b, ST16], and to nonhomogeneous equations [TT17] are also available.

The WED approach fits into the general class of global-in-time variational methods for evolution equations, see [MS11] for some survey. Among the many options, we shall minimally mention the celebrated BrÉZIS-EKELAND-NAYROLES principle [BE76, BE76b, Nay76], its generalization in the frame of self-dual Lagrangian theory [Gho09], and its extensions to doubly nonlinear [Ste08, Vis11], maximal-monotone [Vis08, Vis13], and pseudo-monotone flows [Vis15]. 
In this paper we aim to extend the result from [MS11] to gradient flows set in a

$$
\text { complete metric space }(X, \mathrm{~d}) \text {. }
$$

We will thus prove, for a reasonably wide class of driving energy functionals $\phi$, that WED minimizers $u_{\varepsilon}$ of the functional

$$
\mathcal{I}_{\varepsilon}[u]:=\int_{0}^{\infty} \mathrm{e}^{-t / \varepsilon}\left(\frac{1}{2}\left|u^{\prime}\right|^{2}(t)+\frac{1}{\varepsilon} \phi(u(t))\right) \mathrm{d} t,
$$

among a natural class of absolutely continuous curves $u:[0, \infty) \rightarrow X$ satisfying a given initial condition $u(0)=x$, converge (up to subsequences) to curves of maximal slope for $\phi$, characterized by

$$
-(\phi \circ u)^{\prime}(t)=\frac{1}{2}\left|u^{\prime}\right|^{2}(t)+\frac{1}{2}|\partial \phi|^{2}(u(t))=\left|u^{\prime}\right|^{2}(t)=|\partial \phi|^{2}(u(t)) \quad \text { for a.a. } t \in(0, \infty) .
$$

In (1.5), the metric derivative $\left|u^{\prime}\right|(t)$ has to be understood as the metric surrogate of the norm $\left|u^{\prime}(t)\right|$, and it indeed replaces the latter in the associated WED functional (1.4); $|\partial \phi|$ denotes the metric slope of the energy $\phi ;(1.5)$ is the differential characterization of Curves of Maximal Slope when $|\partial \phi|$ is a strong upper gradient.

In this way, we show that the WED approach shares the same features of the well-known Minimizing Movement scheme [AGS08], which relies on a recursive minimization of a functional combining distance and energy. Notice that in a metric space an underlying linear structure is missing, as well as the Euler equation (1.3). Moreover, the dissipation term provided by the metric velocity $\left|u^{\prime}\right|^{2}$ is not required to be a quadratic form on a linear tangent space, so that even in a linear framework (e.g. in a Banach space) the resulting evolution equation is doubly nonlinear.

One of the basic feature of the WED approach is that it does not directly involve the distance $d$ but it relies on the notion of length (and quadratic action) of a curve. We thus hope that our metric strategy could be extended to more general cases of length structures, where the length of a curve may be strongly affected by the geometry of the sublevels of the functional $\phi$.

In what follows, we briefly recapitulate the challenges attached to this analysis, and the main ideas underlying the proof of our main result. This discussion will also make apparent how big the leap is between the Hilbert and the metric theory.

The analysis in Hilbert vs. metric spaces. The starting point in the proof of [MS11, Thm. 1.1] in a Hilbert space $H$ is the observation that, by the the direct method of calculus of variations and the $\lambda$-convexity of $\phi$, the WED functional $\mathcal{I}_{\varepsilon, T}(1.2)$ admits a unique minimizer $u_{\varepsilon}:[0, T] \rightarrow H$ among all trajectories starting from a given initial datum $x \in H$. A suitable smoothing argument allows the authors to show that $u_{\varepsilon}$ fulfills the Euler-Lagrange equation (1.3), supplemented with the initial condition $u_{\varepsilon}(0)=x$ and the additional Neumann boundary condition $\varepsilon u_{\varepsilon}^{\prime}(T)=0$ at the final time $T$. In fact, (1.3) is the elliptic (in time) regularization of the original gradient flow (1.1). Its role is twofold: first of all, from (1.3) it is possible to deduce the key estimate

$$
\varepsilon\left\|u_{\varepsilon}^{\prime \prime}\right\|_{L^{2}(0, T ; H)}+\varepsilon^{1 / 2}\left\|u_{\varepsilon}^{\prime}\right\|_{L^{\infty}(0, T ; H)}+\left\|u_{\varepsilon}^{\prime}\right\|_{L^{2}(0, T ; H)}+\left\|\xi_{\varepsilon}\right\|_{L^{2}(0, T ; H)} \leq C,
$$

with $\xi_{\varepsilon}$ a selection in $\partial \phi\left(u_{\varepsilon}\right)$ satisfying (1.3). Secondly, it is in (1.3) that, exploiting the above estimates, it is possible to pass to the limit as $\varepsilon \downarrow 0$, proving the convergence of the curves $\left(u_{\varepsilon}\right)_{\varepsilon}$ to the (unique) solution of (1.1).

The arguments in [MS11] clearly rely on two structural properties available in Hilbert spaces: the linear setting and the quadratic norm. It is far from obvious how to replicate them in the metric context, where the gradient flow equation (1.1) is formulated by means of the notion of Curve of Maximal Slope (1.5).

In our metric setup, once that the existence of minimizers for (1.4) (among all curves starting from a given initial datum $x \in X$ ) has been proved, a nontrivial challenge is to provide new metric insights, taking the place of the Euler-Lagrange equation (1.3).

A first piece of information can be obtained by taking inner variations with respect to time (namely, perturbations by time rescalings) of a minimizer $u_{\varepsilon}$ for $\mathcal{I}_{\varepsilon}$, which lead (cf. Proposition 
3.7) to the metric inner variation equation

$$
\frac{\mathrm{d}}{\mathrm{d} t}\left(\phi\left(u_{\varepsilon}(t)\right)-\frac{\varepsilon}{2}\left|u_{\varepsilon}^{\prime}\right|^{2}(t)\right)=-\left|u_{\varepsilon}^{\prime}\right|^{2}(t) \quad \text { in } \mathcal{D}^{\prime}(0, \infty) .
$$

However, even in a finite dimensional framework it is clear that equation (1.6) is much weaker than the former (1.3) and does not contain enough information to characterize the evolution.

We will borrow the second crucial idea from the theory of Optimal control and Hamilton-Jacobi equations, cf. e.g. [BCD97]. In this direction, the key step will be to work with the value functional associated with the minimum problem for $\mathcal{I}_{\varepsilon}$, namely

$$
V_{\varepsilon}(x):=\min _{u \in \operatorname{AC}([0, \infty) ; X), u(0)=x} \mathcal{I}_{\varepsilon}[u]
$$

Variational properties of the value function and its gradient flow. From the WED point of view, the value function $V_{\varepsilon}$ plays a crucial role, which can be compared to the importance of the Yosida approximation

$$
\phi_{\varepsilon}(x):=\min _{y \in X}\left(\frac{1}{2 \varepsilon} \mathrm{d}^{2}(x, y)+\phi(y)\right)
$$

in the Minimizing Movement approach. In order to illustrate the main ideas in a simpler situation, in the following lines we will keep to the finite-dimensional framework $X=\mathbb{R}^{n}$ and consider a smooth energy $\phi: \mathbb{R}^{n} \rightarrow \mathbb{R}$. In this setting, a classical result from the theory of Optimal Control (cf., e.g., [BCD97, Chap. III, Prop. 2.5]), ensures that the value function for the infinite-horizon minimum problem complies with the Dynamic Programming Principle. Namely, there holds

$$
V_{\varepsilon}(x)=\min _{u \in \operatorname{AC}\left([0, \infty) ; \mathbb{R}^{n}\right), u(0)=x}\left(\int_{0}^{T} \mathrm{e}^{-t / \varepsilon}\left(\frac{1}{2}\left|u^{\prime}(t)\right|^{2}+\frac{1}{\varepsilon} \phi(u(t))\right) \mathrm{d} t+V_{\varepsilon}(u(T)) \mathrm{e}^{-T / \varepsilon}\right)
$$

for all $T>0$, and every minimizer $u_{\varepsilon}$ for (1.4) is also a minimizer for the minimum problem (1.9), whence

$$
\left.V_{\varepsilon}(x)=\int_{0}^{T} \mathrm{e}^{-t / \varepsilon}\left(\frac{1}{2}\left|u_{\varepsilon}^{\prime}(t)\right|^{2}+\frac{1}{\varepsilon} \phi\left(u_{\varepsilon}(t)\right)\right) \mathrm{d} t+V_{\varepsilon}\left(u_{\varepsilon}(T)\right)\right) \mathrm{e}^{-T / \varepsilon} \quad \text { for all } T>0 .
$$

We recall the interpretation of formula (1.9) provided in [BCD97], viz. that, to achieve the minimum cost it is necessary and sufficient to:

(1) let the system evolve in an arbitrary finite interval $[0, T]$, along an arbitrary trajectory $u$

(2) pay the corresponding cost, i.e. $\int_{0}^{T} \mathrm{e}^{-t / \varepsilon}\left(\frac{1}{2}\left|u^{\prime}(t)\right|^{2}+\frac{1}{\varepsilon} \phi(u(t))\right) \mathrm{d} t$

(3) pay what remains to pay in a optimal way, i.e. $\left.V_{\varepsilon}(u(T))\right) \mathrm{e}^{-T / \varepsilon}$

(4) minimize over all possible trajectories.

As we will see now, the Dynamic Programming Principle (1.9) is the milestone of our analysis. Indeed, from (1.10) one deduces that for every $0 \leq s \leq t$

$$
\begin{aligned}
\int_{s}^{t} \mathrm{e}^{-r / \varepsilon}\left(\frac{1}{2}\left|u_{\varepsilon}^{\prime}(r)\right|^{2}+\frac{1}{\varepsilon} \phi\left(u_{\varepsilon}(r)\right)\right) \mathrm{d} r & \left.\left.=V_{\varepsilon}\left(u_{\varepsilon}(s)\right)\right) \mathrm{e}^{-s / \varepsilon}-V_{\varepsilon}\left(u_{\varepsilon}(t)\right)\right) \mathrm{e}^{-t / \varepsilon} \\
& \left.=-\int_{s}^{t} \frac{\mathrm{d}}{\mathrm{d} r}\left(V_{\varepsilon}\left(u_{\varepsilon}(r)\right)\right) \mathrm{e}^{-r / \varepsilon}\right) \mathrm{d} r \\
& =-\int_{s}^{t} \frac{\mathrm{d}}{\mathrm{d} r}\left(V_{\varepsilon}\left(u_{\varepsilon}(r)\right)\right) \mathrm{e}^{-r / \varepsilon} \mathrm{d} r+\int_{s}^{t} \frac{1}{\varepsilon} \mathrm{e}^{-r / \varepsilon} V_{\varepsilon}\left(u_{\varepsilon}(r)\right) \mathrm{d} r
\end{aligned}
$$

Rearranging terms and using the Lebesgue Theorem we then conclude the Fundamental identity

$$
-\frac{\mathrm{d}}{\mathrm{d} t} V_{\varepsilon}\left(u_{\varepsilon}(t)\right)=\frac{1}{2}\left|u_{\varepsilon}^{\prime}(t)\right|^{2}+\frac{1}{\varepsilon} \phi\left(u_{\varepsilon}(t)\right)-\frac{1}{\varepsilon} V_{\varepsilon}\left(u_{\varepsilon}(t)\right) \quad \text { for a.a. } t \in(0, \infty) .
$$

In fact, (1.11) can be combined with another consequence of the Dynamic Programming Principle (cf. [BCD97, Chap. III, Thm. 2.12]), i.e.. that the value function $V_{\varepsilon}$ fulfills the Hamilton-Jacobi equation

$$
\frac{1}{\varepsilon} V_{\varepsilon}(x)+\mathcal{H}\left(x, \mathrm{D} V_{\varepsilon}(x)\right)=0 \quad \text { in } \mathbb{R}^{n}
$$


where the Hamiltonian $\mathcal{H}: \mathbb{R}^{n} \times \mathbb{R}^{n} \rightarrow \mathbb{R}$ is defined by

$$
\mathcal{H}(x, p):=\sup _{v \in R^{n}}\left(-x \cdot v-\frac{|v|^{2}}{2}-\frac{1}{\varepsilon} \phi(x)\right)=\frac{1}{2}|p|^{2}-\frac{1}{\varepsilon} \phi(x) .
$$

Hence, (1.12) yields

$$
\frac{1}{2}\left|\mathrm{D} V_{\varepsilon}(x)\right|^{2}=\frac{1}{\varepsilon} \phi(x)-\frac{1}{\varepsilon} V_{\varepsilon}(x) \quad \text { in } \mathbb{R}^{n} .
$$

Combining (1.11) and (1.13) we thus arrive at the crucial relation

$$
-\frac{\mathrm{d}}{\mathrm{d} t} V_{\varepsilon}\left(u_{\varepsilon}(t)\right)=\frac{1}{2}\left|u_{\varepsilon}^{\prime}(t)\right|^{2}+\frac{1}{2}\left|\mathrm{D} V_{\varepsilon}\left(u_{\varepsilon}(t)\right)\right|^{2} \quad \text { for a.a. } t \in(0, \infty),
$$

i.e. we conclude that any WED minimizer $u_{\varepsilon}$ fulfills the gradient flow equation driven by the value function $V_{\varepsilon}$. Since the latter can be thought as an approximation of the energy functional $\phi$ as $\varepsilon \downarrow 0$ (as in the case of the Yosida regularization (1.8)), this argument suggests that a possible way to prove the convergence of the WED minimizers to a solution of gradient flow for $\phi$, is to pass to the limit directly in (1.14). We will follow this idea in the metric setting.

Main results. In the metric framework we will suppose that the energy functional $\phi$ complies with the lower semicontinuity-coercivity-compactness (LSCC) conditions, by now standard in the variational approach to metric gradient flows, cf. [AGS08, Chap. II], namely

Lower Semicontinuity: $\phi$ is sequentially lower semicontinuous;

Compactness: Every d-bounded set contained in a sublevel of $\phi$ is relatively sequentially compact;

Coercivity: There exists $u_{*} \in X$ and constants $\mathrm{A}, \mathrm{B} \geq 0$ such that $\phi(u) \geq-\mathrm{Bd}^{2}\left(u, u_{*}\right)-\mathrm{A}$ for all $u \in X$.

In fact, throughout the paper we will work with a generalized version of the above conditions, featuring an interplay between the topology induced by the metric $\mathrm{d}$ and a second topology $\sigma$, cf. LSCC Property 2.5 ahead.

Our first result, Theorem 3.2, ensures that, under the LSCC Property 2.5 there exists a minimizer for the WED functional $\mathcal{I}_{\varepsilon}$ among all trajectories starting from a given datum $x \in \mathrm{D}(\phi)$. Its proof relies on an integral compactness criterion, Theorem 2.6 ahead, which establishes suitable compactness properties for any sequence $\left(u_{n}\right)_{n} \subset \mathrm{AC}_{\text {loc }}([0, \infty) ; X)$ such that

$$
\sup _{n} \int_{J}\left|u_{n}^{\prime}\right|^{2}(t) \mathrm{d} t \leq C, \quad \sup _{n} \int_{J} \phi\left(u_{n}(t)\right) \mathrm{d} t \leq C,
$$

for every compact interval $J \subset[0, \infty)$. Observe that (1.15) are indeed the estimates that can be deduced from $\sup _{n \in \mathbb{N}} \mathcal{I}_{\varepsilon}\left[u_{n}\right] \leq C$.

It can be shown that the Dynamic Programming Principle also holds for the metric value function (1.7). Then, the calculations leading to (1.11) carry over to the metric setting, allowing us to conclude the metric analogue of (1.11) for any WED minimizer $u_{\varepsilon}$, cf. Proposition 4.3. Namely, there holds

$$
-\frac{\mathrm{d}}{\mathrm{d} t} V_{\varepsilon}\left(u_{\varepsilon}(t)\right)=\frac{1}{2}\left|u_{\varepsilon}^{\prime}\right|^{2}(t)+\frac{1}{\varepsilon} \phi\left(u_{\varepsilon}(t)\right)-\frac{1}{\varepsilon} V_{\varepsilon}\left(u_{\varepsilon}(t)\right) \quad \text { for a.a. } t \in(0, \infty) .
$$

Relation (1.16) is a cornerstone in the proof of our main result, Theorem 3.6, here recalled in a slightly simplified form, and without specifying the topology involved in the definition of the lower semicontinuous relaxation $\left|\partial^{-} \phi\right|$ of the local slope $|\partial \phi|$ :

Theorem 1.1. Assume the LSCC Property 2.5 and that the relaxed slope $\left|\partial^{-} \phi\right|$ is an upper gradient for $\phi$. Let $\bar{u} \in \mathrm{D}(\phi)$ and $\left(u_{\varepsilon}\right)_{\varepsilon}$ be a family of curves minimizing $\mathcal{I}_{\varepsilon}$ among all trajectories starting from $\bar{u}$.

Then, for any vanishing sequence $\left(\varepsilon_{k}\right)_{k}$ the curves $\left(u_{\varepsilon_{k}}\right)_{k}$ pointwise converge on $[0, \infty)$, up to a subsequence, to a curve of maximal slope for $\phi$ (with respect to $\left.\left|\partial^{-} \phi\right|\right)$.

Let us highlight that the convergence of WED minimizers holds under the very same conditions ensuring the existence of curves of maximal slope for $\phi$, cf. [AGS08]. 
We now briefly comment on the main ideas underlying the proof of Theorem 1.1: It is in (1.16) that we shall pass to the limit, as $k \rightarrow \infty$, to show that any limit curve $u$ of the sequence $\left(u_{\varepsilon_{k}}\right)_{k}$ is a curve of maximal slope. The basic ingredients for taking the limit in an integrated version of (1.16) will be the lower estimates

$$
\liminf _{k \rightarrow \infty} V_{\varepsilon_{k}}\left(u_{\varepsilon_{k}}(t)\right) \geq \phi(u(t)) \quad \text { for all } t \in[0, \infty),
$$

and

$$
\liminf _{k \rightarrow \infty} \int_{0}^{t} \frac{1}{\varepsilon_{k}}\left(\phi\left(u_{\varepsilon_{k}}(s)\right)-V_{\varepsilon_{k}}\left(u_{\varepsilon_{k}}(s)\right)\right) \mathrm{d} s \geq \int_{0}^{t} \frac{1}{2}|\partial \phi|^{2}(u(s)) \mathrm{d} s \quad \text { for all } t \in[0, \infty) .
$$

Finally, observe that relation (1.16) does contain the information that any WED minimizer $u_{\varepsilon}$ is a curve of maximal slope for the value function $V_{\varepsilon}$ : this is shown in Theorem 4.6, revealing the upper gradient properties of the quantity $G_{\varepsilon}=\sqrt{2 \frac{\phi-V_{\varepsilon}}{\varepsilon}}$, see also Thm. A.6 in the Appendix. What is more, if in addition $\phi$ is $\lambda$-geodesically convex, it can be shown (Theorem 7.1 ) that the following Hamilton-Jacobi identity holds

$$
\frac{1}{\varepsilon} \phi(u)-\frac{1}{\varepsilon} V_{\varepsilon}(u)=\left|\tilde{\partial} V_{\varepsilon}\right|^{2}(u) \quad \text { for all } u \in \mathrm{D}(\phi)
$$

(with $\left|\tilde{\partial} V_{\varepsilon}\right|$ a slightly modified version of the local slope of $V_{\varepsilon}$ ). Hence, (1.16) reads

$$
-\frac{\mathrm{d}}{\mathrm{d} t} V_{\varepsilon}\left(u_{\varepsilon}(t)\right)=\frac{1}{2}\left|u_{\varepsilon}^{\prime}\right|^{2}(t)+\frac{1}{2}\left|\tilde{\partial} V_{\varepsilon}\right|^{2}\left(u_{\varepsilon}(t)\right) \quad \text { for a.a. } t \in(0, \infty),
$$

and the analogy with (1.14) in the Banach framework is complete.

We will show that (1.17) holds pointwise; it would be interesting to study its formulation in other contexts, e.g. in connection with the recently developed theory of viscosity solutions to Hamilton-Jacobi equations in metric spaces, cf. [AF14, GS15, GHN15]. Notice however that when $(X, \mathrm{~d})$ is not locally compact (as it mostly happens for infinite-dimensional dynamics), the LSCC assumptions prevent the continuity of the driving energy $\phi$ and of the value function $V_{\varepsilon}$. This gives rise to technical issues in the viscosity approach.

Remark 1.2. All the results of the present paper could be easily extended to more general dissipation terms, induced by $p$-powers, $1<p<\infty$, or by superlinear convex functions $\psi$ : $[0, \infty) \rightarrow[0, \infty)$ as in [RMS08, Sect. 2.4]; they correspond to WED functionals of the form

$$
\mathcal{I}_{\varepsilon}^{\psi}[u]:=\int_{0}^{\infty} \mathrm{e}^{-t / \varepsilon}\left(\psi\left(\left|u^{\prime}\right|(t)\right)+\frac{1}{\varepsilon} \phi(u(t))\right) \mathrm{d} t
$$

and to the metric gradient flow

$$
-(\phi \circ u)^{\prime}(t)=\psi\left(\left|u^{\prime}\right|(t)\right)+\psi^{*}(|\partial \phi|(u(t))) \quad \text { for a.a. } t \in(0, \infty) .
$$

However, in order to keep the presentation simpler, we will only focus on the case $p=2, \psi(v)=$ $\frac{1}{2} v^{2}$.

Plan of the paper. In Section 2, after recalling some basic notions on metric gradient flows, we fix the metric-topological setup of our results, precisely state our assumptions on the energy functional $\phi$, and prove some preliminary results, among which the compactness criterion in Thm. 2.6 .

$\underline{\text { Section } 3}$ is devoted to the minimization of the WED functional $\mathcal{I}_{\varepsilon}$ : the existence of minimizers is shown in Thm. 3.2, and the metric inner variation equation established in Prop. 3.7.

A thorough analysis of the properties of the value function $V_{\varepsilon}$ is carried out throughout Section 4, where in particular we prove that any WED minimizer is a curve of maximal slope for $V_{\varepsilon}$.

In Section 5 we finally pass to the limit as $\varepsilon \downarrow 0$ in the gradient flow equation for $V_{\varepsilon}$, and conclude the proof of Theorem 3.6.

Under the additional $\lambda$-geodesic convexity of $\phi$, in Section 6 we prove finer results on WED minimizers $u_{\varepsilon}$ : in particular, we show that, for every fixed $\varepsilon>0$, the mapping $t \mapsto \phi\left(u_{\varepsilon}(t)\right)$ 
enjoys continuity, monotonicity, and convexity properties akin to those holding for the function $t \mapsto \phi(u(t))$ whenever $u$ is a curve of maximal slope for $\phi$.

Keeping the $\lambda$-convexity assumption, in $\underline{\text { Section } 7}$ we establish the Hamilton-Jacobi identity (1.17) in Thm. 7.1.

Section 8 shows some applications of our results to gradient flows of nonconvex functonals in Hilbert and Banach spaces (Sec. 8.1), and to a class of curves of maximal slope in Wasserstein spaces of probability measures (Sec. 8.2).

Finally, in Appendix A we introduce a Finsler-type metric on $X$, induced by $\phi$, which will provide further insight into the properties of $V_{\varepsilon}$.

Notation 1.3 (General notation). Throughout the paper, we shall use the symbols $c, c^{\prime}, C, C^{\prime}$, etc., whose meaning may vary even within the same line, to denote various positive constants depending only on known quantities. Furthermore, the symbols $I_{i}, i=0,1, \ldots$, will be used as place-holders for several integral terms (or sums of integral terms) involved in the various estimates: we warn the reader that we will not be self-consistent with the numbering, so that, for instance, the symbol $I_{1}$ will occur several times with different meanings.

\section{SETUP AND PRELIMINARY RESUlTS}

In this section, in order to make the paper as self-contained as possible, we first collect some basic definitions and results from the theory of gradient flows in metric spaces in Sec. 2.1, referring to [AGS08] for all details, as well as some auxiliary results on the reparameterization of curves (Sec. 2.2). In Sec. 2.3 we then state the basic lower semicontinuity/coercivity assumptions on the energy $\phi$ under which we shall prove the main results in this paper, also relying on the compactness criterion provided by Theorem 2.6 (Sec. 2.4). We conclude by fixing some results on the exponential measure

$$
\mu_{\varepsilon}:=\frac{1}{\varepsilon} \mathrm{e}^{-t / \varepsilon} \mathscr{L}^{1},
$$

and the induced weighted Sobolev spaces, that will turn out to be useful in order to study the properties of the WED functional $\mathcal{I}_{\varepsilon}(1.4)$, cf. Sec. 2.5.

Throughout the paper we will assume that

$$
(X, \mathrm{~d}) \text { is a complete metric space. }
$$

2.1. Recaps on gradient flows in metric spaces.

Absolutely continuous curves and metric derivative. Let $I$ be an interval of $\mathbb{R}$. We say that a curve $u: I \rightarrow X$ belongs to $\operatorname{AC}^{p}(I ; X), p \in[1, \infty]$, if there exists $m \in L^{p}(I)$ such that

$$
\mathrm{d}(u(s), u(t)) \leq \int_{s}^{t} m(r) \mathrm{d} r \quad \text { for all } s, t \in I, s \leq t .
$$

For $p=1$, we simply write $\mathrm{AC}(I ; X)$ and speak of absolutely continuous curves. The case $p=\infty$ corresponds to Lipschitz curves. As usual, $\mathrm{AC}_{\text {loc }}^{p}(I ; X)$ will denote the set of curves $u: I \rightarrow X$ which belong to $\mathrm{AC}^{p}(J ; X)$ for every compact interval $J \subset I$.

It can be proved (see e.g. [AGS08, Sec. 1.1]) that for all $u \in \operatorname{AC}^{p}(I ; X)$, the limit

$$
\left|u^{\prime}\right|(t)=\lim _{s \rightarrow t} \frac{\mathrm{d}(u(s), u(t))}{|t-s|}
$$

exists for a.a. $t \in I$. We will refer to it as the metric derivative of $u$ at $t$. The map $t \mapsto\left|u^{\prime}\right|(t)$ belongs to $L^{p}(I)$ and it is minimal within the class of functions $m \in L^{p}(I)$ fulfilling (2.1).

A distinguished class of Lipschitz curves in $\mathrm{AC}^{\infty}([0,1] ; X)$ is provided by minimal, constantspeed, geodesics (for short, geodesics): they are curves $\gamma:[0,1] \rightarrow X$ satisfying

$$
\mathrm{d}(\gamma(s), \gamma(t))=|t-s| \mathrm{d}(\gamma(0), \gamma(1)) \quad \text { for every } s, t \in[0,1] ;
$$

in particular a geodesic $\gamma$ satisfies $\left|\gamma^{\prime}\right|(t) \equiv \mathrm{d}(\gamma(0), \gamma(1))$ for every $t \in(0,1)$. 
Local (descending) slope and moderated upper gradient of a l.s.c. functional. Let

$$
\begin{gathered}
\phi: X \rightarrow(-\infty, \infty] \text { be lower semicontinuous with proper domain } \\
\qquad D(\phi):=\{u \in X: \phi(u)<\infty\} \neq \emptyset .
\end{gathered}
$$

The local (descending) slope (see [DGMT80b, AGS08]) of $\phi$ at $u \in D(\phi)$ is defined by

$$
|\partial \phi|(u):=\limsup _{v \rightarrow u} \frac{(\phi(u)-\phi(v))^{+}}{\mathrm{d}(u, v)} .
$$

As already mentioned in the Introduction, we will also consider on the space $X$ a second (Hausdorff) topology $\sigma$, suitably compatible with that induced by the metric d (cf. (2.18) ahead). Accordingly, we will work with the sequentially $\sigma$-lower semicontinuous envelope of the local slope $|\partial \phi|$ along $d$-bounded sequences with bounded energy, namely the relaxed slope

$$
\left|\partial^{-} \phi\right|(u):=\inf \left\{\liminf _{n \rightarrow \infty}|\partial \phi|\left(u_{n}\right): u_{n} \stackrel{\sigma}{\rightarrow} u, \sup _{n}\left(d\left(u_{n}, u\right), \phi\left(u_{n}\right)\right)<\infty\right\} \quad \text { for } u \in D(\phi) .
$$

We recall (cf. [HK98, Che99], [AGS08, Def. 1.2.1]) that a function $g: X \rightarrow[0, \infty]$ is a (strong) upper gradient for the functional $\phi$ if, for every curve $u \in \mathrm{AC}(I ; X)$ the function $g \circ u$ is Borel and there holds

$$
|\phi(u(t))-\phi(u(s))| \leq \int_{s}^{t} g(u(r))\left|u^{\prime}\right|(r) \mathrm{d} r \quad \text { for all } 0<s \leq t .
$$

We now now introduce a slightly weaker notion of upper gradient which is well adapted to gradient flows of functionals that can assume the value $\infty$. Let $p \in[1, \infty]$ : We say that a function $g: X \rightarrow[0, \infty]$ is an $L^{p}$-moderated upper gradient for the functional $\phi$ if for every curve $u \in \mathrm{AC}(I ; X)$ such that, in addition,

$$
\phi \circ u \in L^{p}\left(I ;\left|u^{\prime}\right| \mathscr{L}^{1}\right), \quad g \circ u \in L^{1}\left(I ;\left|u^{\prime}\right| \mathscr{L}^{1}\right)
$$

we still have (2.4). Observe that, whenever $g$ is a strong, or a $L^{p}$-moderated, upper gradient, we have that $\phi \circ u \in \mathrm{AC}(I ; \mathbb{R})$ and

$$
\left|(\phi \circ u)^{\prime}(t)\right| \leq g(u(t))\left|u^{\prime}\right|(t) \quad \text { for a.a. } t \in I .
$$

Curves of Maximal Slope. Let $g: X \rightarrow[0, \infty]$ be an $L^{\infty}$-moderated upper gradient for $\phi$ and let $I=[0, \infty)$. We recall (see [AGS08, Def. 1.3.2, p.32], following [DGMT80b, Amb95]), that a curve $u \in \mathrm{AC}_{\mathrm{loc}}^{2}([0, \infty) ; X)$ is said to be a curve of maximal slope for the functional $\phi$ with respect to $g$ if $\phi \circ u$ is locally bounded and

$$
\text { - }(\phi \circ u)^{\prime}(t)=\left|u^{\prime}\right|^{2}(t)=g^{2}(u(t)) \quad \text { for a.a. } t \in(0, \infty) .
$$

In particular, $\phi \circ u$ is locally absolutely continuous in $[0, \infty), g \circ u \in L_{\mathrm{loc}}^{2}([0, \infty))$, and the energy identity

$$
\frac{1}{2} \int_{s}^{t}\left|u^{\prime}\right|^{2}(r) \mathrm{d} r+\frac{1}{2} \int_{s}^{t} g^{2}(u(r)) \mathrm{d} r+\phi(u(t))=\phi(u(s)) \quad \text { for all } 0 \leq s \leq t,
$$

directly follows. It is interesting that curves of maximal slope w.r.t. $g$ can be characterized by an integral condition: in fact, if a curve $u \in \mathrm{AC}_{\text {loc }}^{2}([0, \infty) ; X)$ with $u(0) \in \mathrm{D}(\phi)$ satisfies

$$
\frac{1}{2} \int_{0}^{t}\left|u^{\prime}\right|^{2}(r) \mathrm{d} r+\frac{1}{2} \int_{0}^{t} g^{2}(u(r)) \mathrm{d} r+\phi(u(t)) \leq \phi(u(0)) \quad \text { for all } t \geq 0
$$

then $u$ fulfills (2.6). Notice that, for any reasonable definition of gradient flow local boundedness of $\phi \circ u$ is not a restrictive a priori assumption: this justifies the restriction to the class of moderated upper gradients in the above definition of curve of maximal slope. 
Geodesically $\lambda$-convex functionals. A remarkable case in which the local slope is a (strong) upper gradient occurs when (cf. [AGS08, Thm. 2.4.9]) the functional $\phi$ is geodesically $\lambda$-convex for some $\lambda \in \mathbb{R}$, i.e.

for all $v_{0}, v_{1} \in \mathrm{D}(\phi)$ there exists a geodesic $\gamma:[0,1] \rightarrow X \quad$ with $\gamma(0)=v_{0}, \gamma(1)=v_{1}$, and

$$
\phi(\gamma(t)) \leq(1-t) \phi\left(v_{0}\right)+t \phi\left(v_{1}\right)-\frac{\lambda}{2} t(1-t) \mathrm{d}^{2}\left(v_{0}, v_{1}\right) \text { for all } 0 \leq t \leq 1 .
$$

The following result subsumes [AGS08, Cor. 2.4.10, Lemma 2.4.13, Thm. 2.4.9].

Proposition 2.1. Let $\phi: X \rightarrow(-\infty, \infty]$ be $\mathrm{d}$-lower semicontinuous and $\lambda$-geodesically convex for some $\lambda \in \mathbb{R}$. Then, the local slope $|\partial \phi|$ is lower semicontinuous and admits the representation

$$
|\partial \phi|(u)=\sup _{v \neq u}\left(\frac{\phi(u)-\phi(v)}{\mathrm{d}(u, v)}+\frac{\lambda}{2} \mathrm{~d}(u, v)\right)^{+} \quad \text { for all } u \in \mathrm{D}(\phi) .
$$

Furthermore, $|\partial \phi|$ is a (strong) upper gradient, and therefore an $L^{p}$-moderated upper gradient for every $p \in[1, \infty]$.

2.2. Length and energy reparameterization. We recall here a few standard results on reparameterization of curves, from which we will also derive a useful criterion for a functional to be an upper gradient. Lemma 2.2 below shall be also used in the proof of Lemma A.2 ahead.

Lemma 2.2 (Length and Energy reparameterization). Let $\mathfrak{g}: X \rightarrow[0, \infty)$ be a Borel function and let $\vartheta \in \mathrm{AC}([a, b] ; X)$ be a curve with $\int_{a}^{b}\left|\vartheta^{\prime}\right|(t) \mathrm{d} t=L$. The reparameterized curve $\tilde{\vartheta}:[0, L] \rightarrow X$

$$
\tilde{\vartheta}(r):=\vartheta(\kappa(r)) \quad \text { with } \kappa(r):=\inf \left\{t \in[a, b]: \int_{a}^{t}\left|\vartheta^{\prime}\right|(s) \mathrm{d} s=r\right\} \text { for all } r \in[0, L],
$$

is 1-Lipschitz, $\kappa:[0, L] \rightarrow[0,1]$ is continuous, nondecreasing and surjective (so that $\tilde{\vartheta}$ has the same support as $\vartheta$ with the same initial and final points), and

$$
\begin{aligned}
& \left|\tilde{\vartheta}^{\prime}\right|(r)=1 \quad \text { for } \mathscr{L}^{1} \text {-a.e. } r \in[0, L], \\
& \int_{\kappa\left(r_{0}\right)}^{\kappa\left(r_{1}\right)} \mathfrak{g}(\vartheta(t))\left|\vartheta^{\prime}\right|(t) \mathrm{d} t=\int_{r_{0}}^{r_{1}} \mathfrak{g}(\tilde{\vartheta}(r)) \mathrm{d} r \quad \text { for all } 0 \leq r_{0}<r_{1} \leq L .
\end{aligned}
$$

Similarly, if $S:=\int_{a}^{b} \frac{1}{\mathfrak{g}(\vartheta(t))}\left|\vartheta^{\prime}\right|(t) \mathrm{d} t<\infty$, the reparametrized curve $\vartheta_{\mathfrak{g}}:[0, S] \rightarrow X$

$$
\vartheta_{\mathfrak{g}}(s):=\vartheta\left(\kappa_{\mathfrak{g}}(s)\right), \quad \kappa_{\mathfrak{g}}(s):=\inf \left\{t \in[a, b]: \int_{a}^{t} \frac{1}{\mathfrak{g}(\vartheta(t))}\left|\vartheta^{\prime}\right|(r) \mathrm{d} r=s\right\}, \quad s \in[0, S],
$$

belongs to $\mathrm{AC}([0, S] ; X)$, has the same support as $\vartheta$ with the same initial and final points, and satisfies

$$
\mathfrak{g}\left(\vartheta_{\mathfrak{g}}(s)\right)=\left|\vartheta_{\mathfrak{g}}^{\prime}\right|(s) \quad \text { a.e. in }[0, S] .
$$

In particular, if $\int_{a}^{b} \mathfrak{g}(\vartheta(t))\left|\vartheta^{\prime}\right|(t) \mathrm{d} t<\infty$ we have $\vartheta_{\mathfrak{g}} \in \mathrm{AC}^{2}([0, S] ; X)$ and

$$
\int_{0}^{S} \mathfrak{g}^{2}\left(\vartheta_{\mathfrak{g}}(s)\right) \mathrm{d} s=\int_{0}^{S}\left|\vartheta_{\mathfrak{g}}^{\prime}\right|^{2}(s) \mathrm{d} s=\int_{0}^{S} \mathfrak{g}\left(\vartheta_{\mathfrak{g}}(s)\right)\left|\vartheta_{\mathfrak{g}}^{\prime}\right|(s) \mathrm{d} s=\int_{0}^{1} \mathfrak{g}(\vartheta(t))\left|\vartheta^{\prime}\right|(t) \mathrm{d} t .
$$

Proof. The first part of the statement is a direct application of the 1-dimensional area formula, see e.g. [AT00, Rem. 4.2.2].

In order to prove the second part, based on the construction (2.11) of the curve $\tilde{\vartheta}$, let us define s : $[0, L] \rightarrow[0, \infty)$ by

$$
\mathrm{s}(r):=\int_{0}^{r} \frac{1}{\mathfrak{g}(\tilde{\vartheta}(z))} \mathrm{d} z=\int_{0}^{\kappa(r)} \frac{1}{\mathfrak{g}(\vartheta(t))}\left|\vartheta^{\prime}\right|(t) \mathrm{d} t, \quad S:=\mathrm{s}(L) .
$$


Since $\mathfrak{g} \circ \tilde{\vartheta}<\infty$ a.e. in $(0, L)$ by $(2.12)$, we have $\mathrm{s}^{\prime}(r)=\frac{1}{\mathfrak{g}(\tilde{\vartheta}(r))}>0$ for almost all $r \in(0, L)$, so that the map $\mathrm{s}$ is strictly increasing hence invertible, with inverse $r:[0, S] \rightarrow[0, L]$ satisfying $\kappa_{\mathfrak{g}}(s)=\kappa(\mathrm{r}(s))$ and $\vartheta_{\mathfrak{g}}(s)=\vartheta\left(\kappa_{\mathfrak{g}}(s)\right)=\vartheta(\kappa(\mathrm{r}(s)))=\tilde{\vartheta}(\mathrm{r}(s))$. Since

$$
\left|\vartheta_{\mathfrak{g}}^{\prime}\right|(s)=\frac{\left|\tilde{\vartheta}^{\prime}\right|(\mathrm{r}(s))}{\mathrm{s}^{\prime}(\mathrm{r}(s))}=\mathfrak{g}(\tilde{\vartheta}(\mathrm{r}(s)))=\mathfrak{g}\left(\vartheta_{\mathfrak{g}}(s)\right) \quad \text { for a.a. } s \in(0, S),
$$

we get (2.14); a further application of the change of variable formula yields (2.15).

As a first application of the previous Lemma we have the following criterion that will be applied in Section 4.2 to prove that a certain quantity is a moderated upper gradient for the value function associated with the WED functional, cf. Theorem 4.6 ahead.

Corollary 2.3 (A criterion for upper gradients). Let $\phi: X \rightarrow(-\infty, \infty]$ be a l.s.c. functional and let $g: X \rightarrow[0, \infty]$ be a Borel map such that for every curve $\vartheta \in \mathrm{AC}^{2}([a, b] ; X)$ with $\phi \circ \vartheta \in L^{2 p}(a, b)$ there holds

$$
|\phi(\vartheta(b))-\phi(\vartheta(a))| \leq \frac{1}{2} \int_{a}^{b}\left(\left|\vartheta^{\prime}\right|^{2}(t)+g(\vartheta(t))\right) \mathrm{d} t
$$

Then $g$ is a $L^{p}$-moderated upper gradient for $\phi$.

Proof. In order to prove that $g$ is an upper gradient, it is not restrictive to check condition (2.4) in the case when $I=[0,1]$ and $s=0 t=1$. We also assume $p<\infty$, as the modifications in the case $p=\infty$ are obvious.

Let us fix a curve $\vartheta \in \mathrm{AC}([0,1] ; X)$ such that $\phi \circ \vartheta \in L^{p}\left(0,1 ;\left|\vartheta^{\prime}\right| \mathscr{L}^{1}\right)$ and $g \circ \vartheta \in L^{1}\left(0,1 ;\left|\vartheta^{\prime}\right| \mathscr{L}^{1}\right)$. Setting $g_{\varepsilon}(x):=g(x)+\varepsilon(1 \vee|\phi(x)|)^{p}$, we can apply the second part of Lemma 2.2 to find a reparametrized curve $\vartheta_{\varepsilon}:=\vartheta_{g_{\varepsilon}} \in \mathrm{AC}^{2}\left(\left[0, S_{\varepsilon}\right] ; X\right)$ corresponding to $g_{\varepsilon}$ such that

$$
\int_{0}^{S_{\varepsilon}} g_{\varepsilon}^{2}\left(\vartheta_{\varepsilon}(s)\right) \mathrm{d} s=\int_{0}^{S_{\varepsilon}}\left|\vartheta_{\varepsilon}^{\prime}\right|^{2}(s) \mathrm{d} s=\int_{0}^{S_{\varepsilon}} g_{\varepsilon}\left(\vartheta_{\varepsilon}(s)\right)\left|\vartheta_{\varepsilon}^{\prime}\right|(s) \mathrm{d} s=\int_{0}^{1} g_{\varepsilon}(\vartheta(t))\left|\vartheta^{\prime}\right|(t) \mathrm{d} t .
$$

Applying (2.17) to the curve $\vartheta_{\varepsilon}$ (notice that $\phi \circ \vartheta_{\varepsilon} \in L^{2 p}\left(0, S_{\varepsilon}\right)$ since $\left|\phi\left(\vartheta_{\varepsilon}\right)\right|^{p} \leq g_{\varepsilon}\left(\vartheta_{\varepsilon}\right)$ ), we obtain

$$
\begin{aligned}
\mid \phi(\vartheta(1))-\phi & (\vartheta(0))|=| \phi\left(\vartheta_{\varepsilon}\left(S_{\varepsilon}\right)\right)-\phi\left(\vartheta_{\varepsilon}(0)\right) \mid \leq \frac{1}{2} \int_{0}^{S_{\varepsilon}}\left(\left|\vartheta_{\varepsilon}^{\prime}\right|^{2}(s)+g\left(\vartheta_{\varepsilon}(s)\right)\right) \mathrm{d} s \\
\leq & \frac{1}{2} \int_{0}^{S_{\varepsilon}}\left(\left|\vartheta_{\varepsilon}^{\prime}\right|^{2}(s)+g_{\varepsilon}\left(\vartheta_{\varepsilon}(s)\right)\right) \mathrm{d} s=\int_{0}^{1} g_{\varepsilon}(\vartheta(t))\left|\vartheta^{\prime}\right|(t) \mathrm{d} t \\
& =\int_{0}^{1} g(\vartheta(t))\left|\vartheta^{\prime}\right|(t) \mathrm{d} t+\varepsilon \int_{0}^{1}(1 \vee \phi(\vartheta(t)))^{p}\left|\vartheta^{\prime}\right|(t) \mathrm{d} t
\end{aligned}
$$

Passing to the limit as $\varepsilon \downarrow 0$ we conclude the desired estimate (2.4).

2.3. A general metric-topological framework for gradient flows. Throughout the paper, we will always assume that $(X, \mathrm{~d})$ is a complete metric space endowed with an auxiliary Hausdorff topology $\sigma$ on $X$, that satisfies the following compatibility conditions:

(MT1) $d$ is sequentially lower semicontinuous w.r.t. the product topology induced by $\sigma$ on $X \times X$ :

$$
\left(u_{n}, v_{n}\right) \stackrel{\sigma}{\rightarrow}(u, v) \Rightarrow \liminf _{n \rightarrow \infty} \mathrm{d}\left(u_{n}, v_{n}\right) \geq \mathrm{d}(u, v)
$$

(MT2) for every $\sigma$-open set $U$ and every $x \in U$

there exist a $\sigma$-open neighborhood $V$ of $x$ and $\delta>0$ such that $\mathrm{d}(y, V)<\delta \Rightarrow y \in U$. (2.18b)

We call $(X, \mathrm{~d}, \sigma)$ a compatible metric-topological space. Notice that (MT2) in particular shows that $\sigma$ is weaker than the topology induced by the distance $d$. The possibility to work with two possibly different topologies allows for a wider applicability of the theory, as the following examples show.

Remark 2.4 (Examples of compatibile metric-topological structures).

(E1) The above condition is obviously satisfied in the simplest case in which $\sigma$ coincides with the topology induced by $\mathrm{d}$. 
(E2) Another interesting example is provided by a topology $\sigma$ induced by another distance $\mathrm{d}^{\prime}$ satisfying $\mathrm{d}^{\prime}(x, y) \leq C \mathrm{~d}(x, y)$ for every $x, y \in X$ : this is the typical situation when $X$ is a Banach space continuously imbedded in a Banach space $Y$ and $\mathrm{d}, \mathrm{d}^{\prime}$ are the distances induced by the norms of $X$ and $Y$ respectively.

(E3) In the previous example, $Y$ could also be a Fréchet space: consider e.g. the case when $X=L^{p}(\Omega), \Omega$ open subset of some Euclidean space $\mathbb{R}^{m}$, and $\sigma$ is the topology of $L_{\mathrm{loc}}^{p}(\Omega)$, induced by the distance

$$
\mathrm{d}^{\prime}(f, g):=\sum_{n=1}^{\infty} 2^{-n}\left(\|f-g\|_{L^{p}\left(K_{n}\right)} \wedge 1\right)
$$

where $K_{n} \Subset \Omega$ is a nondecreasing sequence of compact subsets invading $\Omega$.

(E4) As a further example, one can consider the weak topology in a Banach space $X$, when $\mathrm{d}$ is the distance induced by its norm. This example also highlights that it is interesting to deal with possibly non-metrizable topologies.

(E5) Transport distances provide another important example: we can consider $\mathcal{X}=\mathscr{P}_{p}(X)$ endowed with the $p$-Wasserstein distance $W_{p}, p \in[1, \infty)$, and the topology $\sigma$ of weak convergence of probability measures, cf. e.g. [AGS08, Chap. 7].

Property 2.5 (Standard lower semicontinuity-coercivity-compactness (LSCC) assumptions). We say that the proper functional $\phi: X \rightarrow(-\infty, \infty]$ satisfies the standard assumptions if the following properties hold:

Lower Semicontinuity: $\phi$ is $\sigma$-sequentially lower semicontinuous on $\mathrm{d}$-bounded sets:

$$
\sup _{n, m} \mathrm{~d}\left(u_{n}, u_{m}\right)<\infty, u_{n} \stackrel{\sigma}{\rightarrow} u \Rightarrow \liminf _{n \rightarrow \infty} \phi\left(u_{n}\right) \geq \phi(u) ;
$$

Compactness: Every d-bounded set contained in a sublevel of $\phi$ is relatively $\sigma$-sequentially compact:

$$
\begin{aligned}
& \text { if }\left(u_{n}\right)_{n} \subset X \text { with } \sup _{n} \phi\left(u_{n}\right)<\infty, \sup _{n, m} \mathrm{~d}\left(u_{n}, u_{m}\right)<\infty, \\
& \text { then }\left(u_{n}\right)_{n} \text { admits a } \sigma \text {-convergent subsequence. }
\end{aligned}
$$

Coercivity: There exists $u_{*} \in X$ and constants $\mathrm{A}, \mathrm{B} \geq 0$ such that

$$
\phi(u) \geq-\mathrm{Bd}^{2}(u, v)-\mathrm{Q}(v), \text { where } \mathrm{Q}(v):=\mathrm{Bd}^{2}\left(v, u_{*}\right)+\mathrm{A} \text { for every } u, v \in X .
$$

Notice that if $\phi$ satisfies

$$
\phi(u) \geq-\mathrm{a}-\mathrm{bd}^{2}\left(u, u_{*}\right) \quad \text { for every } u \in X,
$$

for some $\mathrm{a}, \mathrm{b} \geq 0$ then $(2.19 \mathrm{c})$ holds with $\mathrm{A}:=\mathrm{a}$ and $\mathrm{B}:=2 \mathrm{~b}$.

The simplest situation in which Property 2.5 holds is provided by a functional $\phi$ whose sublevels $\{v \in X: \phi(v) \leq c\}$ are compact in $(X, \mathrm{~d})$; in this case we can choose $\sigma$ to be the topology induced by $d$.

2.4. An integral compactness criterion. In this section we adapt to our setting a compactness result for sequences of absolutely continuous curves drawn from [RS03, Rmk. Extension 1, Thm. 4.12]. First and foremost, we shall apply it to show with Theorem 3.2 the existence of minimizers for the WED functional, relying on a pointwise equicontinuity estimate. Since we have it at our disposal, we can provide a simpler and more direct proof of Thm. 2.6 than that in [RS03].

Theorem 2.6. Let $I$ be an interval of $\mathbb{R}$ and let us assume that $\phi: X \rightarrow(-\infty, \infty]$ satisfies the standard LSCC Property 2.5. If $\left(u_{n}\right)_{n} \subset \mathrm{AC}_{\text {loc }}^{2}(I ; X)$ is a sequence satisfying

$$
\sup _{n} \int_{J}\left|u_{n}^{\prime}\right|^{2}(t) \mathrm{d} t<\infty, \quad \sup _{n} \int_{J} \phi\left(u_{n}(t)\right) \mathrm{d} t<\infty \quad \text { for every compact interval } J \subset I,
$$

and $\left(u_{n}\left(t_{0}\right)\right)_{n}$ is bounded for some $t_{0} \in I$, then there exists a limit function $u \in \operatorname{AC}_{\mathrm{loc}}^{2}(I ; X)$ and a subsequence $k \mapsto n_{k}$ such that

$$
u_{n_{k}}(t) \stackrel{\sigma}{\rightarrow} u(t) \text { for every } t \in I \text { as } k \uparrow \infty,
$$




$$
\begin{gathered}
\left|u_{n_{k}}^{\prime}\right| \rightarrow v \quad \text { in } L_{\mathrm{loc}}^{2}(I) \text { as } k \uparrow \infty, \quad v \geq\left|u^{\prime}\right| \mathscr{L}^{1} \text {-a.e. in } I, \\
\liminf _{k \rightarrow \infty} \int_{J} \phi\left(u_{n_{k}}(t)\right) \zeta(t) \mathrm{d} t \geq \int_{J} \phi(u(t)) \zeta(t) \mathrm{d} t
\end{gathered}
$$

for every nonnegative $\zeta \in \mathrm{C}(I)$ and every compact interval $J \subset I$.

Proof. By a standard diagonal argument, it is not restrictive to assume that $I=[a, b]$ is a compact interval. Since the sequence $\left(u_{n}\left(t_{0}\right)\right)_{n}$ is uniformly bounded, we can assume that there exists a constant $C>0$ such that

$$
\int_{a}^{b}\left|u_{n}^{\prime}\right|^{2}(t) \mathrm{d} t \leq C, \quad \sup _{t \in[a, b]} \mathrm{d}\left(u_{n}(t), u_{*}\right) \leq C, \quad \int_{a}^{b} \psi\left(u_{n}(t)\right) \mathrm{d} t \leq C \quad \text { for every } n \in \mathbb{N},
$$

where

$$
\psi(w):=\phi(w)+\operatorname{Bd}^{2}\left(w, u_{*}\right)+\mathbf{A} \geq 0, \quad w \in X,
$$

and $u_{*}, \mathrm{~A}, \mathrm{~B}$ are given by $(2.19 \mathrm{c})$. The first integral bound of (2.25) also yields

$$
\mathrm{d}\left(u_{n}(t), u_{n}(s)\right) \leq \int_{s}^{t}\left|u_{n}^{\prime}\right|(r) \mathrm{d} r \leq \sqrt{C|t-s|} \text { for every } \quad s, t \in I, s \leq t, n \in \mathbb{N} .
$$

Up to extracting a suitable subsequence, it is not restrictive to assume that $\left|u_{n}^{\prime}\right| \rightarrow v$ as $n \rightarrow \infty$ in $L^{2}(a, b)$.

Let $J_{m}, m \in \mathbb{N}$, be a countable basis of open sets in $(a, b)$. We want to find a family of sequences indexed by $m \in \mathbb{N}$, that we represent by a map $(m, k) \mapsto n(m, k) \in \mathbb{N}$, and points $t_{m} \in J_{m}$ with $t_{m} \neq t_{m^{\prime}}$ if $m \neq m^{\prime}$, such that

- for every $m \in \mathbb{N}$ the sequence $k \mapsto n(m+1, k)$ is an increasing subsequence of $k \mapsto n(m, k)$

- $k \mapsto u_{n(m, k)}\left(t_{m}\right)$ is converging to some $w_{m} \in X$ w.r.t. $\sigma$ as $k \rightarrow \infty$.

We argue by induction w.r.t. $m$. When $m=0$ we simply set $n(0, k):=k$. Assuming that the sequence $k \mapsto n(m, k)$ is given for some $m \in \mathbb{N}$, Fatou's lemma yields

$$
\int_{a}^{b} \liminf _{k \rightarrow \infty} \psi\left(u_{n(m, k)}(t)\right) \mathrm{d} t \leq C
$$

so that $\liminf _{k \rightarrow \infty} \psi\left(u_{n(m, k)}(t)\right)<\infty$ for $\mathscr{L}^{1}$-a.e. $t \in[a, b]$. In particular, since $\mathscr{L}^{1}\left(J_{m+1}\right)>0$, there exists a point $t_{m+1} \in J_{m+1} \backslash\left\{t_{1}, \cdots, t_{m}\right\}$ such that $\liminf _{k \rightarrow \infty} \psi\left(u_{n(m, k)}\left(t_{m+1}\right)\right)<\infty$ and therefore by (2.19b) we can find a subsequence $k \mapsto n(m+1, k)$ of $k \mapsto n(m, k)$ and a limit point $w_{m+1} \in X$ such that $u_{n(m+1, k)}\left(t_{m+1}\right) \stackrel{\sigma}{\rightarrow} w_{m+1}$.

By a Cantor diagonal argument, we conclude that the sequence $k \mapsto n_{k}:=n(k, k)$ satisfies $u_{n_{k}}\left(t_{m}\right) \stackrel{\sigma}{\rightarrow} w_{m}$ for every $m \in \mathbb{N}$. Since $J_{m}$ is a countable basis of open intervals in $(a, b)$, the set $D=\left\{t_{m}: m \in \mathbb{N}\right\}$ is countable and dense in $[a, b]$ : we can then define a function $u: D \rightarrow X$ by setting $u\left(t_{m}\right):=w_{m}$.

Now we can argue as in [AGS08, Prop. 3.3.1] to conclude, by a careful use of the compatibility conditions (2.18a) and (2.18b) between $\mathrm{d}$ and $\sigma$. In fact, passing to the limit in (2.27) thanks to (2.18a) we get

$$
\mathrm{d}(u(t), u(s)) \leq \int_{s}^{t} v(r) \mathrm{d} r \leq \sqrt{C|t-s|} \text { for every } \quad s, t \in D, s \leq t .
$$

By (2.28) and the completeness of $X$, we can extend $u$ to a curve (still denoted by $u$ ) defined on $I$ and still satisfying estimate (2.28) for every $s, t \in I$. In particular $u \in \operatorname{AC}^{2}(I ; X)$ and $\left|u^{\prime}\right| \leq v$, so that (2.23) is proved.

In order to prove convergence (2.22), we pick an arbitrary point $t \in I$ and a $\sigma$-neighborhood $U$ of $u(t)$. Let then $\delta>0$ and $V$ be as in the compatibility assumption (MT2) (with $x=u(t)$ ). Since $u$ is d-continuous (and therefore also $\sigma$-continuous) we can then find a point $s \in D$ such that $C|t-s|<\delta^{2}$ and $u(s) \in V$. Since $u_{n_{k}}(s) \stackrel{\sigma}{\rightarrow} u(s)$ as $k \uparrow \infty$ we can also find $\bar{k}$ sufficiently big such that $u_{n_{k}}(s) \in V$ for every $k \geq \bar{k}$. Since $\mathrm{d}\left(u_{n_{k}}(s), u_{n_{k}}(t)\right) \leq \sqrt{C|t-s|}<\delta$ for every $k \in \mathbb{N}$ by (2.27), we deduce by $(2.18 \mathrm{~b})$ that $u_{n_{k}}(t) \in U$ for every $k \geq \bar{k}$. 
The lower estimate (2.24) then follows by Fatou's Lemma and the fact that $\phi \circ u_{n}$ is uniformly bounded from below by a constant. The latter boundedness ensues from the $\mathrm{d}$-uniform boundedness of $u_{n}$ given by $(2.25)$, combined with (2.19c).

We can refine the pointwise convergence in $(2.22)$ by showing that the sequence $\left(u_{n_{k}}\right)_{k}$ is in fact converging in the compact-open topology induced by $\sigma$. When $\sigma$ is metrizable, this implies the locally uniform convergence of $\left(u_{n_{k}}\right)_{k}$. In fact, this is a general property of any sequence of $\mathrm{d}$-equicontinuous functions that pointwise converge in the $\sigma$-topology.

Lemma 2.7. Let $\left(u_{k}\right)_{k \in \mathbb{N}} \subset \mathrm{C}(I ; X)$ be a sequence of locally $\mathrm{d}$-equicontinuous functions pointwise converging to $u$ pointwise in the $\sigma$-topology as $k \uparrow \infty$. Then $\left(u_{k}\right)_{k}$ converges to $u$ in the compactopen topology induced by $\sigma$.

Proof. Let us consider an arbitrary open neighborhood $\mathcal{U}$ of $u$ in the compact-open topology: this corresponds to a finite collection of compact sets $K_{m} \subset I$ and corresponding $\sigma$-open sets $U_{m} \subset X$ such that $u\left(K_{m}\right) \subset U_{m}, m \in M:=\{1,2, \cdots, \bar{m}\}$. For every $t \in K_{m}$ let $V(t)$ be a $\sigma$-open neighborhood of $u(t)$ and $\delta(t)>0$ satisfying (2.18b) for $x=u(t)$ and $U=U_{m}$.

We then find $\eta(t)>0$ with

$$
\mathrm{d}\left(u_{k}(r), u_{k}(s)\right) \leq \delta(t) / 2 \quad \text { for every } r, s \in \cup_{m} K_{m} \text { with }|s-r| \leq \eta(t) \text { and } k \in \mathbb{N},
$$

and we set

$$
W(t):=u^{-1}(V(t)) \cap B(t, \eta(t)) \cap K_{m}, \quad \text { where } B(t, \eta):=\{s \in I:|s-t|<\eta\} .
$$

Since $\left\{W(t): t \in K_{m}\right\}$ is an open covering of $K_{m}$, we can find a finite subcovering $\left\{W(t): t \in J_{m}\right\}$ corresponding to a finite set $J_{m}=\left\{t_{m, 1}, \cdots, t_{m, \bar{h}(m)}\right\}$ of points in $K_{m}$. We define $\delta_{m, h}:=\delta\left(t_{m, h}\right)$, $\eta_{m, h}:=\eta\left(t_{m, h}\right)$ and consider the new collection of compact sets $K_{m, h}:=\overline{W\left(t_{m, h}\right)} \subset I$ and points $t_{m, h} \in K_{m, h}$ indexed by integers in $N:=\{(m, h) \in \mathbb{N} \times \mathbb{N}: m \leq \bar{m}, h \leq \bar{h}(m)\}$ with the property that

$$
\begin{gathered}
\bigcup_{1 \leq h \leq \bar{h}(m)} K_{m, h}=K_{m}, \quad K_{m, h} \subset B\left(t_{m, h}, \eta_{m, h}\right), \\
u\left(K_{m, h}\right) \subset V\left(t_{m, h}\right) \quad \text { for every }(m, h) \in N .
\end{gathered}
$$

The neighborhood $\mathcal{U}$ can then be represented as the set of $\sigma$-continuous curves $w: I \rightarrow X$ with $w\left(K_{m, h}\right) \subset U_{m}$ for every $(m, h) \in N$.

Arguing as in the proof of the Theorem 2.6, we can find $\bar{k}$ sufficiently big such that $u_{k}\left(t_{m, h}\right) \in$ $V\left(t_{m, h}\right)$ for every $k \geq \bar{k}$ and $(m, h) \in N$. Since $K_{m, h} \subset B\left(t_{m, h}, \eta_{m, h}\right)$, the equicontinuity estimate $(2.27)$ and $(2.18 \mathrm{~b})$ yield $u_{k}\left(K_{m, h}\right) \subset U_{m}$, thus $u_{k} \in \mathcal{U}$ for every $k \geq \bar{k}$, which concludes the proof of the convergence of $\left(u_{k}\right)_{k}$.

2.5. The exponential measure and weighted Sobolev spaces. In this section we quickly recall a few basic properties of the Sobolev spaces $W^{1,2}\left(0, \infty ; \mu_{\varepsilon}\right)$ induced by the probability measure

$$
\mu_{\varepsilon}:=\frac{\mathrm{e}^{-t / \varepsilon}}{\varepsilon} \mathscr{L}^{1} \text { i.e. } \quad \int_{0}^{\infty} \zeta(t) \mathrm{d} \mu_{\varepsilon}(t):=\int_{0}^{\infty} \zeta(t) \frac{\mathrm{e}^{-t / \varepsilon}}{\varepsilon} \mathrm{d} t .
$$

We say that $w \in W^{1, p}\left(0, \infty ; \mu_{\varepsilon}\right), p \in[1, \infty)$, if $w \in W_{\mathrm{loc}}^{1, p}((0, \infty))$ and

$$
\int_{0}^{\infty}\left(|w(t)|^{p}+\left|w^{\prime}(t)\right|^{p}\right) \mathrm{d} \mu_{\varepsilon}(t)<\infty .
$$

Denoting by $\tilde{v}$ the continuous representative of the function $v$, we easily check that $\tilde{v} \in \operatorname{AC}_{\text {loc }}^{p}([0, \infty) ; \mathbb{R})$ and for $v, w \in W^{1,2}\left(0, \infty ; \mu_{\varepsilon}\right)$ the following integration by parts formula holds

$$
\int_{a}^{b} \varepsilon v w^{\prime} \mathrm{d} \mu_{\varepsilon}=\int_{a}^{b}\left(-\varepsilon v^{\prime}+v\right) w \mathrm{~d} \mu_{\varepsilon}+\mathrm{e}^{-b / \varepsilon} \tilde{v}(b) \tilde{w}(b)-\mathrm{e}^{-a / \varepsilon} \tilde{v}(a) \tilde{w}(a) \text { for all } 0 \leq a<b<\infty .
$$


Lemma 2.8. If $w \in \mathrm{AC}_{\text {loc }}([0, \infty) ; \mathbb{R})$ with $\int_{0}^{\infty}\left|w^{\prime}(t)\right| \mathrm{d} \mu_{\varepsilon}(t)<\infty$ then $w \in W^{1,1}\left(0, \infty ; \mu_{\varepsilon}\right)$ and

$$
\begin{aligned}
& w(0)+\varepsilon \int_{0}^{T} w^{\prime}(t) \mathrm{d} \mu_{\varepsilon}(t)=w(T) \mathrm{e}^{-T / \varepsilon}+\int_{0}^{T} w(t) \mathrm{d} \mu_{\varepsilon}(t) \quad \text { for every } T>0, \\
& w(0)+\varepsilon \int_{0}^{\infty} w^{\prime}(t) \mathrm{d} \mu_{\varepsilon}(t)=\int_{0}^{\infty} w(t) \mathrm{d} \mu_{\varepsilon}(t) .
\end{aligned}
$$

In particular, if $u \in W^{1, q}\left(0, \infty ; \mu_{\varepsilon}\right)$ and $v \in W^{1, p}\left(0, \infty ; \mu_{\varepsilon}\right)$ for a couple of conjugate exponents $p, q \in[1, \infty]$, then

$$
\tilde{u}(0) \tilde{v}(0)+\int_{0}^{\infty} \varepsilon u v^{\prime} \mathrm{d} \mu_{\varepsilon}=\int_{0}^{\infty}\left(-\varepsilon u^{\prime}+u\right) v \mathrm{~d} \mu_{\varepsilon} .
$$

Proof. Formula (2.33) follows from (2.32) for $v \equiv 1$. Setting $W(t):=\int_{0}^{t}\left|w^{\prime}(r)\right| \mathrm{d} r$, (2.33) yields for every $T>0$

$$
\varepsilon \int_{0}^{T}\left|w^{\prime}(t)\right| \mathrm{d} \mu_{\varepsilon}(t)=\varepsilon \int_{0}^{T} W^{\prime}(t) \mathrm{d} \mu_{\varepsilon}(r)=\int_{0}^{T} W(r) \mathrm{d} \mu_{\varepsilon}(r)+\mathrm{e}^{-T / \varepsilon} W(T) .
$$

Passing to the limit as $T \uparrow \infty$ we get $W \in L^{1}\left(0, \infty ; \mu_{\varepsilon}\right)$ and, since

$$
|w(t)| \leq|w(0)|+W(t)
$$

we deduce that $w \in L^{1}\left(0, \infty ; \mu_{\varepsilon}\right)$. Since $\mathrm{e}^{-t / \varepsilon} W(t)$ has finite integral, its limit set as $t \rightarrow \infty$ should contain 0. Therefore, from (2.37) we gather $\lim _{t \rightarrow \infty} \mathrm{e}^{-t / \varepsilon} W(t)=\lim _{t \rightarrow \infty} \mathrm{e}^{-t / \varepsilon} w(t)=0$. Passing to the limit as $T \uparrow \infty$ in (2.33) we get (2.34).

Finally, (2.35) follows by applying (2.34) to $w:=u v$.

Starting from (2.35) it is easy to check that a function $w \in L_{\text {loc }}^{1}(0, \infty)$ belongs to $W_{\text {loc }}^{1,1}(0, \infty)$ if and only if there exists $g \in L_{\text {loc }}^{1}(0, \infty)$ such that

$$
\int_{0}^{\infty} w\left(-\varepsilon \xi^{\prime}+\xi\right) \mathrm{d} \mu_{\varepsilon}=\int_{0}^{\infty} \varepsilon g \xi \mathrm{d} \mu_{\varepsilon} \quad \text { for every } \xi \in \mathrm{C}_{c}^{\infty}(0, \infty)
$$

and in this case $w^{\prime}=g$ in the distributional sense.

In Lemma 2.9 below we compute the sharp constant for the Poincaré inequality for real functions in $W^{1,2}\left(0, \infty ; \mu_{\varepsilon}\right)$ that vanish at 0 : it will play a crucial role in the next section in order to derive suitable bounds on infimizing sequences for the WED functional.

Lemma 2.9. For every function $w \in \mathrm{AC}_{\text {loc }}^{2}([0, \infty) ; \mathbb{R})$ with $w(0)=0$, every $\varepsilon>0$ and every $T \in(0, \infty]$ we have

$$
\int_{0}^{T}\left|w^{\prime}(t)\right|^{2} \mathrm{~d} \mu_{\varepsilon}(t) \geq \frac{1}{4 \varepsilon^{2}} \int_{0}^{T}|w(t)|^{2} \mathrm{~d} \mu_{\varepsilon}(t)
$$

In particular, if $\lambda \in\left(-\infty, 1 / 4 \varepsilon^{2}\right)$ and $\left(w_{n}\right)_{n} \subset \mathrm{AC}_{\text {loc }}^{2}([0, \infty) ; \mathbb{R})$ is a sequence satisfying $w_{n}(0)=0$ and

$$
\sup _{n \in \mathbb{N}} \int_{0}^{\infty}\left(\left|w_{n}^{\prime}(t)\right|^{2}-\lambda\left|w_{n}(t)\right|^{2}\right) \mathrm{d} \mu_{\varepsilon}(t) \leq C<\infty \quad \text { for every } n \in \mathbb{N}
$$

then there exists an increasing subsequence $k \mapsto n_{k}$ such that, as $k \rightarrow \infty,\left(w_{n_{k}}\right)_{k}$ converges to $w \in \mathrm{AC}_{\text {loc }}^{2}([0, \infty) ; \mathbb{R})$ locally uniformly, $w_{n_{k}}^{\prime} \rightarrow w^{\prime}$ weakly in $L^{2}\left(0, \infty ; \mu_{\varepsilon}\right)$, and for every $\eta \in$ $\left(-\infty, 1 / 4 \varepsilon^{2}\right]$

$$
\liminf _{k \rightarrow \infty} \int_{0}^{\infty}\left(\left|w_{n_{k}}^{\prime}(t)\right|^{2}-\eta\left|w_{n_{k}}(t)\right|^{2}\right) \mathrm{d} \mu_{\varepsilon}(t) \geq \int_{0}^{\infty}\left(\left|w^{\prime}(t)\right|^{2}-\eta|w(t)|^{2}\right) \mathrm{d} \mu_{\varepsilon}(t) .
$$

Proof. Let us first prove (2.39). For every $\alpha, \beta \geq 0$ we have

$$
\begin{aligned}
\int_{0}^{T}\left|\alpha w^{\prime}-\beta w\right|^{2} \mathrm{~d} \mu_{\varepsilon} & =\int_{0}^{T}\left(\alpha^{2}\left(w^{\prime}\right)^{2}+\beta^{2} w^{2}\right) \mathrm{d} \mu_{\varepsilon}-\alpha \beta \int_{0}^{T}\left(w^{2}\right)^{\prime} \mathrm{d} \mu_{\varepsilon} \\
& =\alpha^{2} \int_{0}^{T}\left|w^{\prime}\right|^{2} \mathrm{~d} \mu_{\varepsilon}+\left(\beta^{2}-\frac{\alpha \beta}{\varepsilon}\right) \int_{0}^{T} w^{2} \mathrm{~d} \mu_{\varepsilon}-\frac{\alpha \beta}{\varepsilon} \mathrm{e}^{-T / \varepsilon} w^{2}(T),
\end{aligned}
$$


where the second equality follows from applying (2.33) to $w^{2}$. Choosing $\alpha=1, \beta=\frac{1}{2 \varepsilon}$ we get (2.39) for finite $T>0$; passing to the limit as $T \uparrow \infty$ in (2.42) and arguing as in the previous Lemma we obtain

$$
\int_{0}^{\infty}\left|w^{\prime}\right|^{2} \mathrm{~d} \mu_{\varepsilon}=\left(1-\alpha^{2}\right) \int_{0}^{\infty}\left|w^{\prime}\right|^{2} \mathrm{~d} \mu_{\varepsilon}+\left(\frac{\alpha \beta}{\varepsilon}-\beta^{2}\right) \int_{0}^{\infty} w^{2} \mathrm{~d} \mu_{\varepsilon}+\int_{0}^{\infty}\left|\alpha w^{\prime}-\beta w\right|^{2} \mathrm{~d} \mu_{\varepsilon} .
$$

Choosing $\alpha<1$ and $\beta>0$ with $\alpha \beta / \varepsilon-\beta^{2}>\lambda$, (2.40) yields that $\left(w_{n}\right)_{n}$ is uniformly bounded in $W^{1,2}\left(0, \infty ; \mu_{\varepsilon}\right)$. By standard weak compactness we obtain a subsequence $\left(w_{n_{k}}\right)_{k}$ weakly converging to some limit $w$ in $W^{1,2}\left(0, \infty ; \mu_{\varepsilon}\right)$, so that $w_{n_{k}} \rightarrow w$ and $w_{n_{k}}^{\prime} \rightarrow w^{\prime}$ weakly in $L^{2}\left(0, \infty ; \mu_{\varepsilon}\right)$ as $k \rightarrow \infty$. The lower estimate (2.41) then follows from (2.43) by choosing $\alpha=1$ and $\beta / \varepsilon-\beta^{2}=\eta$.

Remark 2.10. The optimality of the constant $\frac{1}{4 \varepsilon^{2}}$ on the right-hand side of the inequality $(2.39)$ can be easily checked by considering the sequence $w_{n}(t)=(1 \wedge(n-|t-n|) \vee 0) \mathrm{e}^{-t / 2 \varepsilon}$.

\section{The WeD functional, its minimization, And the main CONVERgence Result}

Let us introduce the functional $\ell_{\varepsilon}: X \times[0, \infty) \rightarrow \mathbb{R}$

$$
\ell_{\varepsilon}(u, v):=\frac{\varepsilon}{2} v^{2}+\phi(u) .
$$

In this section we will investigate the following variational problem.

Problem 3.1 (The $\varepsilon$-WED variational problem). Given $\varepsilon>0$ and $\bar{u} \in X$, minimize the weighted energy-dissipation functional

$$
\mathcal{I}_{\varepsilon}[u]:=\int_{0}^{\infty} \ell_{\varepsilon}\left(u(t),\left|u^{\prime}\right|(t)\right) \mathrm{d} \mu_{\varepsilon}(t)=\int_{0}^{\infty}\left(\frac{\varepsilon}{2}\left|u^{\prime}\right|^{2}(t)+\phi(u(t))\right) \mathrm{d} \mu_{\varepsilon}(t),
$$

over all trajectories $u$ in

$$
\mathscr{C}_{\varepsilon}(\bar{u}):=\left\{u \in \operatorname{AC}_{\mathrm{loc}}^{2}([0, \infty) ; X): u(0)=\bar{u}, \quad \int_{0}^{\infty}\left|u^{\prime}\right|^{2}(t) \mathrm{d} \mu_{\varepsilon}(t)<\infty\right\} .
$$

We will denote by $\mathcal{M}_{\varepsilon}(\bar{u})$ the (possibly empty) set of minimizers of (3.2) in $\mathscr{C}_{\varepsilon}(\bar{u})$.

Even though we will mainly focus on the WED formulation in $(0, \infty)$, it will also be useful to consider a localized version of Problem 3.1: we fix a time $T>0$ and we simply restrict the functional $\mathcal{I}_{\varepsilon}$ to curves which are constant in $[T, \infty)$; we thus introduce

$$
\mathscr{C}_{\varepsilon, T}(\bar{u}):=\left\{u \in \mathrm{AC}_{\mathrm{loc}}^{2}([0, \infty) ; X): u(0)=\bar{u}, \quad u(t) \equiv u(T) \quad \text { in }[T, \infty)\right\},
$$

which is a closed subset of $\mathscr{C}_{\varepsilon}(\bar{u})$ and could also be identified with $\operatorname{AC}^{2}([0, T] ; X)$; we have the obvious inclusions

$$
\mathscr{C}_{\varepsilon, T_{1}}(\bar{u}) \subset \mathscr{C}_{\varepsilon, T_{2}}(\bar{u}) \subset \mathscr{C}_{\varepsilon}(\bar{u})=\mathscr{C}_{\varepsilon, \infty}(\bar{u}) \quad \text { whenever } \quad 0<T_{1}<T_{2}<\infty .
$$

Notice that

$$
\mathcal{I}_{\varepsilon}[u]=\int_{0}^{T} \ell_{\varepsilon}\left(u(t),\left|u^{\prime}\right|(t)\right) \mathrm{d} \mu_{\varepsilon}(t)+\mathrm{e}^{-T / \varepsilon} \phi(u(T)) \quad \text { if } u \in \mathscr{C}_{\varepsilon, T}(\bar{u}) .
$$

We will denote by $\mathcal{M}_{\varepsilon, T}(\bar{u})$ the set of minimizers of $\mathcal{I}_{\varepsilon}$ in $\mathscr{C}_{\varepsilon, T}(\bar{u})$.

3.1. Well-posedness and existence of minimizers of Problem 3.1. First of all, in the metrictopological framework of Section 2.3, for $\varepsilon>0$ sufficiently small (depending on the constant B in $(2.19 \mathrm{c})$ ), we address the well-posedness of Problem 3.1 and the existence of minimizers by assuming that Problem 3.1 is feasible, i.e. that there exists a curve $u \in \mathscr{C}_{\varepsilon}(\bar{u})$ such that $\mathcal{I}_{\varepsilon}[u]<\infty$. This is always the case when $\bar{u} \in \mathrm{D}(\phi)$ : in fact,

the constant curve $u \in \mathscr{C}_{\varepsilon}(\bar{u})$, defined by $\quad u(t) \equiv \bar{u} \quad t \geq 0$, satisfies $\quad \mathcal{I}_{\varepsilon}[u] \leq \phi(\bar{u})$. 
Theorem 3.2. Let us suppose that $\phi$ satisfies the standard LSCC Property 2.5 and that

$$
\frac{1}{16 \varepsilon} \geq \mathrm{B}
$$

Then the integral in (3.2) is well defined (possibly taking the value $\infty$ ) for every $\bar{u} \in X$ and every $u \in \mathscr{C}_{\varepsilon}(\bar{u})$.

Moreover, if Problem 3.1 is feasible (in particular when $\bar{u} \in \mathrm{D}(\phi)$ ) then it admits at least one solution. In this case all the sets $\mathcal{M}_{\varepsilon, T}(\bar{u}), T \in(0, \infty]$, are compact in $\mathscr{C}_{\varepsilon}(\bar{u})$ endowed with the compact-open topology induced by $\sigma$.

We divide the proof of Theorem 3.2 in a few steps, starting from an immediate application of Lemma 2.9. Notice that it is sufficient to consider the case $T=\infty$.

Lemma 3.3. Let $u \in \mathrm{AC}_{\mathrm{loc}}^{2}([0, \infty) ; X), L(t):=\int_{0}^{t}\left|u^{\prime}\right|(r) \mathrm{d} r, \varepsilon>0$, and $T \in(0, \infty]$. Then

$$
\int_{0}^{T}\left|u^{\prime}\right|^{2}(t) \mathrm{d} \mu_{\varepsilon}(t) \geq \frac{1}{4 \varepsilon^{2}} \int_{0}^{T} L^{2}(t) \mathrm{d} \mu_{\varepsilon}(t) \geq \frac{1}{4 \varepsilon^{2}} \int_{0}^{T} \mathrm{~d}^{2}(u(t), u(0)) \mathrm{d} \mu_{\varepsilon}(t) .
$$

In particular, for $\varepsilon>0$ sufficiently small (cf. (3.8)), the integral defining $\mathcal{I}_{\varepsilon}[u]$ in (3.2) is well defined for every $u \in \mathscr{C}_{\varepsilon}(\bar{u})$.

As a further consequence of Lemma 3.3 we provide separate estimates for $\int_{0}^{\infty}\left|u^{\prime}\right|^{2} \mathrm{~d} \mu_{\varepsilon}$ and $\int_{0}^{\infty}(\phi(u))^{+} \mathrm{d} \mu_{\varepsilon}$ for any $u \in \mathscr{C}_{\varepsilon}(\bar{u})$ such that $\mathcal{I}_{\varepsilon}[u]<\infty$ (recall that $(x)^{+}:=x \vee 0$ ). Observe that this in fact requires absorbing the term $-\int_{0}^{\infty} \mathrm{d}^{2}(u(t), u(0)) \mathrm{d} \mu_{\varepsilon}(t)$, which bounds $\int_{0}^{\infty} \phi(u(t)) \mathrm{d} \mu_{\varepsilon}(t)$ from below (cf. (2.19c)), into $\int_{0}^{\infty} \frac{\varepsilon}{2}\left|u^{\prime}\right|^{2} \mathrm{~d} \mu_{\varepsilon}$. It is at this level that (3.9) comes into play.

Lemma 3.4. If $\phi$ satisfies the standard LSCC Property 2.5 and (3.8) holds, then for every $u \in$ $\mathrm{AC}_{\mathrm{loc}}^{2}([0, \infty) ; X)$ there holds

$$
\int_{0}^{\infty}\left(\frac{\varepsilon}{4}\left|u^{\prime}\right|^{2}+(\phi(u(t)))^{+}\right) \mathrm{d} \mu_{\varepsilon}(t) \leq \mathcal{I}_{\varepsilon}[u]+\mathrm{Q}(u(0)) \quad \text { with } \mathrm{Q}(w):=\mathrm{Bd}^{2}\left(w, u_{*}\right)+\mathrm{A} .
$$

Proof. Setting $L(t):=\int_{0}^{t}\left|u^{\prime}\right|(r) \mathrm{d} r$ we write $\mathcal{I}_{\varepsilon}$ as

$$
\mathcal{I}_{\varepsilon}[u]=\frac{\varepsilon}{2} \int_{0}^{\infty}\left(\left|L^{\prime}\right|^{2}-\frac{1}{8 \varepsilon^{2}} L^{2}\right) \mathrm{d} \mu_{\varepsilon}+\int_{0}^{\infty} \psi \mathrm{d} \mu_{\varepsilon}-\mathrm{Q}(u(0)) .
$$

where

$$
\psi(t):=\phi(u(t))+\frac{1}{16 \varepsilon} L^{2}(t)+\mathrm{Q}(u(0)) .
$$

Since $\psi$ is nonnegative thanks to $(2.19 \mathrm{c})$ and $(3.8)$, we have $\psi(t) \geq(\phi(u(t)))^{+}$. On the other hand, we have

$$
\begin{aligned}
\frac{\varepsilon}{2} \int_{0}^{\infty}\left(\left|L^{\prime}\right|^{2}-\frac{1}{8 \varepsilon^{2}} L^{2}\right) \mathrm{d} \mu_{\varepsilon} & =\frac{\varepsilon}{4} \int_{0}^{\infty}\left(\left|L^{\prime}\right|^{2}-\frac{1}{8 \varepsilon^{2}} L^{2}\right) \mathrm{d} \mu_{\varepsilon}+\frac{\varepsilon}{4} \int_{0}^{\infty}\left(\left|L^{\prime}\right|^{2}-\frac{1}{8 \varepsilon^{2}} L^{2}\right) \mathrm{d} \mu_{\varepsilon} \\
& \geq \frac{\varepsilon}{4} \int_{0}^{\infty} \frac{1}{8 \varepsilon^{2}} L^{2} \mathrm{~d} \mu_{\varepsilon}+\frac{\varepsilon}{4} \int_{0}^{\infty}\left|L^{\prime}\right|^{2} \mathrm{~d} \mu_{\varepsilon},
\end{aligned}
$$

where the second estimate follows from (3.9). Then, (3.10) follows.

Corollary 3.5 (Lower semicontinuity and compactness of the functional $\left.\mathcal{I}_{\varepsilon}\right)$. Let $\left(u_{n}\right)_{n}$ be a sequence in $\mathrm{AC}_{\mathrm{loc}}^{2}([0, \infty) ; X)$ such that

$$
\left(u_{n}(0)\right)_{n} \quad \text { is bounded, } \sup _{n \in \mathbb{N}} \mathcal{I}_{\varepsilon}\left[u_{n}\right] \leq C<\infty .
$$

Then there exists an increasing subsequence $k \mapsto n_{k}$ and a limit function $u \in \operatorname{AC}_{\text {loc }}^{2}([0, \infty) ; X)$ such that the conclusions (2.22), (2.23) and (2.24) of Theorem 2.6 hold with $I=[0, \infty)$, and moreover $\mathcal{I}_{\varepsilon}[u] \leq C$. 
Proof. We can apply Theorem 2.6 thanks to estimate (3.10) combined with (3.14). In order to prove that $\mathcal{I}_{\varepsilon}[u] \leq C$ we use the splitting in (3.11) by choosing

$$
L_{n}(t):=\int_{0}^{t}\left|u_{n}^{\prime}\right|(r) \mathrm{d} r, \quad \psi_{n}(t):=\phi\left(u_{n}(t)\right)+\frac{1}{16 \varepsilon} L_{n}^{2}(t)+\mathrm{Q}, \quad \mathrm{Q} \geq \sup _{n} \mathrm{Q}\left(u_{n}(0)\right)
$$

and writing

$$
\mathcal{I}_{\varepsilon}\left[u_{n}\right]=\frac{\varepsilon}{2} \int_{0}^{\infty}\left(\left|L_{n}^{\prime}\right|^{2}-\frac{1}{8 \varepsilon^{2}} L_{n}^{2}\right) \mathrm{d} \mu_{\varepsilon}+\int_{0}^{\infty} \psi_{n} \mathrm{~d} \mu_{\varepsilon}-\mathrm{Q} .
$$

Denoting by $L(t):=\int_{0}^{t} v(r) \mathrm{d} r$ (where $v$ is defined by $(2.23)$ ), we observe that $\left(L_{n_{k}}\right)_{k}$ is pointwise converging to $L \in \mathrm{AC}_{\text {loc }}^{2}(0, \infty ; \mathbb{R})$ with $\left|L^{\prime}\right| \geq\left|u^{\prime}\right| \mathscr{L}^{1}$-a.e. and

$$
\psi(t):=\liminf _{k \rightarrow \infty} \psi_{n_{k}}(t) \geq \phi(u(t))+\frac{1}{16 \varepsilon} L_{n}^{2}(t)+\mathrm{Q} .
$$

Combining (2.41), Fatou's Lemma (which applies since $\psi_{n} \geq 0$ ), and (3.16) we get

$$
C \geq \liminf _{k \rightarrow \infty} \mathcal{I}_{\varepsilon}\left[u_{n_{k}}\right] \geq \frac{\varepsilon}{2} \int_{0}^{\infty}\left(\left|L^{\prime}\right|^{2}-\frac{1}{8 \varepsilon^{2}} L^{2}\right) \mathrm{d} \mu_{\varepsilon}+\int_{0}^{\infty} \psi \mathrm{d} \mu_{\varepsilon}-\mathrm{Q} \geq \mathcal{I}_{\varepsilon}[u] .
$$

The proof of Theorem 3.2 now follows by a simple application of the Direct method of Calculus of Variations.

We conclude this section by stating the main result of the paper on the convergence of sequences of WED minimizers. Its proof is postponed to Section 5.3.

Theorem 3.6. Assume Property 2.5. Let $\left(\bar{u}_{\varepsilon}\right)_{\varepsilon}, \bar{u} \in D(\phi)$ fulfill

$$
\bar{u}_{\varepsilon} \stackrel{\sigma}{\rightarrow} \bar{u}, \quad \sup _{\varepsilon} \mathrm{d}\left(\bar{u}_{\varepsilon}, \bar{u}\right)<\infty, \quad \phi\left(\bar{u}_{\varepsilon}\right) \rightarrow \phi(\bar{u}) \quad \text { as } \varepsilon \downarrow 0 .
$$

For every $\varepsilon>0$, let $u_{\varepsilon} \in \mathcal{M}_{\varepsilon}\left(\bar{u}_{\varepsilon}\right)$.

Then, for any sequence $\left(\varepsilon_{k}\right)_{k}$ with $\varepsilon_{k} \downarrow 0$, there exist a (not relabeled) subsequence and $u \in$ $\mathrm{AC}_{\text {loc }}^{2}([0, \infty) ; X)$, with $u(0)=\bar{u}$, such that

$$
u_{\varepsilon_{k}}(t) \stackrel{\sigma}{\rightarrow} u(t) \quad \forall t \in[0, \infty)
$$

$u(0)=\bar{u}$, and $u$ fulfills

$$
\int_{0}^{t}\left(\frac{1}{2}\left|u^{\prime}\right|^{2}(s)+\frac{1}{2}\left|\partial^{-} \phi\right|^{2}(u(s))\right) \mathrm{d} s+\phi(u(t)) \leq \phi(\bar{u}) \quad \text { for all } t \geq 0 .
$$

Therefore, if in addition $\left|\partial^{-} \phi\right|$ is a $\left(L^{\infty}\right.$-moderated) upper gradient for the functional $\phi, u$ is a curve of maximal slope for $\phi$ w.r.t. $\left|\partial^{-} \phi\right|$.

As already mentioned in the Introduction, a crucial step in the proof of Thm. 3.6 will be to show that WED minimizers are, in a suitable sense discussed at length in Sections 4 and 7 , metric gradient flows for the value functional $V_{\varepsilon}$ (1.7). In turn, a key ingredient for this is the metric inner variation equation (3.20), proved in Section 3.2 below.

3.2. The metric inner variation equation. By taking inner variations of a minimizer of the functional $\mathcal{I}_{\varepsilon}$ we now derive a useful equation.

Proposition 3.7. Let $T \in(0, \infty]$ and let $u$ be a minimizer of $\mathcal{I}_{\varepsilon}$ in $\mathcal{M}_{\varepsilon, T}(\bar{u})$. Then the map $t \mapsto \phi(u(t))-\frac{\varepsilon}{2}\left|u^{\prime}\right|^{2}(t)$ belongs to $W^{1,1}(0, T)\left(W_{\mathrm{loc}}^{1,1}([0, \infty))\right.$ when $\left.T=\infty\right)$ and it fulfills

$$
\frac{\mathrm{d}}{\mathrm{d} t}\left(\phi(u(t))-\frac{\varepsilon}{2}\left|u^{\prime}\right|^{2}(t)\right)=-\left|u^{\prime}\right|^{2}(t) \quad \text { in } \mathcal{D}^{\prime}(0, T)
$$


Proof. Following [GH96, Chap. III], we consider perturbations of $u$ obtained by time rescalings, which we devise by means of the family of smooth diffeomorphisms of $(0, \infty)$

$$
S_{\tau}(t):=t+\tau \xi(t), \quad \xi \in \mathrm{C}_{\mathrm{c}}^{\infty}(0, T) .
$$

Observe that for every $\tau \in \mathbb{R}$ the map $t \mapsto S_{\tau}(t)$ is in $\mathrm{C}^{\infty}(\mathbb{R})$ with smooth inverse $T_{\tau}=S_{\tau}^{-1}$ whenever $|\tau| \cdot \max _{\mathbb{R}}\left|\xi^{\prime}\right|<1$; moreover $S_{\tau}(t)=t$ outside the compact support of $\xi$ in $(0, T)$ and $S_{\tau}((0, T))=(0, T)$. We then define

$$
u_{\tau}:[0, \infty) \rightarrow X \text { by } u_{\tau}(s):=u\left(T_{\tau}(s)\right)=u\left(S_{\tau}^{-1}(s)\right) .
$$

Hence, $u(t)=u_{\tau}\left(S_{\tau}(t)\right)$. Notice that

$$
\left|u_{\tau}^{\prime}\right|(s)=\left|u^{\prime}\right|\left(T_{\tau}(s)\right) T_{\tau}^{\prime}(s)=\frac{\left|u^{\prime}\right|\left(T_{\tau}(s)\right)}{S_{\tau}^{\prime}\left(T_{\tau}(s)\right)} \quad \text { for a.a. } s \in(0, \infty) .
$$

We can compute $\mathcal{I}_{\varepsilon}\left[u_{\tau}\right]$ by applying a standard change of variables

$$
\begin{aligned}
\mathcal{I}\left[u_{\tau}\right] & =\int_{0}^{\infty} \mathrm{e}^{-s / \varepsilon}\left(\frac{1}{2}\left|u_{\tau}^{\prime}\right|^{2}(s)+\frac{1}{\varepsilon} \phi\left(u_{\tau}(s)\right)\right) \mathrm{d} s=\int_{0}^{\infty} \mathrm{e}^{-s / \varepsilon}\left(\frac{1}{2}\left(\frac{\left|u^{\prime}\right|\left(T_{\tau}(s)\right)}{S_{\tau}^{\prime}\left(T_{\tau}(s)\right)}\right)^{2}+\frac{1}{\varepsilon} \phi\left(u_{\tau}(s)\right)\right) \mathrm{d} s \\
& =\int_{0}^{\infty} \mathrm{e}^{-S_{\tau}(t) / \varepsilon}\left(\frac{1}{2} \frac{\left|u^{\prime}\right|^{2}(t)}{S_{\tau}^{\prime}(t)}+\frac{1}{\varepsilon} \phi(u(t)) S_{\tau}^{\prime}(t)\right) \mathrm{d} t
\end{aligned}
$$

and we recover the metric inner variation equation $(3.20)$ by taking the derivative of $\mathcal{I}_{\varepsilon}\left[u_{\tau}\right]$ w.r.t. $\tau$ at the minimum point $\tau=0$. We have

$$
\begin{aligned}
\frac{\mathrm{d}}{\mathrm{d} \tau} \mathcal{I}_{\varepsilon}\left[u_{\tau}\right]= & \int_{0}^{\infty} \mathrm{e}^{-S_{\tau}(t) / \varepsilon}\left(-\frac{1}{\varepsilon} \frac{\partial}{\partial \tau} S_{\tau}(t)\right)\left(\frac{1}{2} \frac{\left|u^{\prime}\right|^{2}(t)}{S_{\tau}^{\prime}(t)}+\frac{1}{\varepsilon} \phi(u(t)) S_{\tau}^{\prime}(t)\right) \mathrm{d} t \\
& +\int_{0}^{\infty} \mathrm{e}^{-S_{\tau}(t) / \varepsilon}\left(-\frac{1}{2} \frac{\left|u^{\prime}\right|^{2}(t)}{\left(S_{\tau}^{\prime}(t)\right)^{2}}+\frac{1}{\varepsilon} \phi(u(t))\right) \frac{\partial}{\partial \tau} S_{\tau}^{\prime}(t) \mathrm{d} t
\end{aligned}
$$

Setting $\tau=0$ and taking into account that

$$
S_{0}(t)=t, \quad S_{\tau}^{\prime}(t)=1+\tau \xi^{\prime}(t), \quad \frac{\partial}{\partial \tau} S_{\tau}(t)=\xi(t), \quad \frac{\partial}{\partial \tau} S_{\tau}^{\prime}(t)=\xi^{\prime}(t),
$$

we conclude that

$$
\begin{aligned}
0=\left.\frac{\mathrm{d}}{\mathrm{d} \tau} \mathcal{I}_{\varepsilon}\left[u_{\tau}\right]\right|_{\tau=0} & =-\int_{0}^{\infty}\left(\frac{1}{2}\left|u^{\prime}\right|^{2}+\frac{1}{\varepsilon} \phi \circ u\right) \xi \mathrm{d} \mu_{\varepsilon}+\int_{0}^{\infty}\left(-\frac{\varepsilon}{2}\left|u^{\prime}\right|^{2}+\phi \circ u\right) \xi^{\prime} \mathrm{d} \mu_{\varepsilon} \\
& =\int_{0}^{\infty}\left[-\left|u^{\prime}\right|^{2} \xi+\left(-\frac{\varepsilon}{2}\left|u^{\prime}\right|^{2}+\phi \circ u\right)\left(\xi^{\prime}-\frac{1}{\varepsilon} \xi\right)\right] \mathrm{d} \mu_{\varepsilon} .
\end{aligned}
$$

Since $\xi \in \mathrm{C}_{c}^{\infty}(0, T)$ is arbitrary, an integration by parts as stated in (2.38) yields (3.20).

Corollary 3.8. Let $u_{\varepsilon, T} \in \mathcal{M}_{\varepsilon, T}(\bar{u}), T \in(0, \infty]$, and let us denote by $\mathcal{V}_{\varepsilon, T}$ the absolutely continuous representative of $t \mapsto \phi\left(u_{\varepsilon, T}(t)\right)-\frac{\varepsilon}{2}\left|u_{\varepsilon, T}^{\prime}\right|^{2}(t)$ in the interval $[0, T]$ (we simply write $u_{\varepsilon}$ and $\mathcal{V}_{\varepsilon}$ when $\left.T=\infty\right)$. Then we have

$$
\mathcal{I}_{\varepsilon}\left[u_{\varepsilon}\right]=\mathcal{V}_{\varepsilon}(0) \quad \text { if } T=\infty
$$

and

$$
\mathcal{I}_{\varepsilon}\left[u_{\varepsilon, T}\right]=\mathcal{V}_{\varepsilon, T}(0)+\mathrm{e}^{-T / \varepsilon}\left(\phi\left(u_{\varepsilon, T}(T)\right)-\mathcal{V}_{\varepsilon, T}(T)\right) \quad \text { if } T<\infty .
$$

Proof. In the case $T=\infty$ the inner variation equation (3.20) gives that the distributional derivative of $\mathcal{V}_{\varepsilon}$ fulfills $\frac{\mathrm{d}}{\mathrm{d} t} \mathcal{V}_{\varepsilon}(t)=-\left|u_{\varepsilon}^{\prime}\right|^{2}(t) \in L^{1}\left(0, \infty ; \mu_{\varepsilon}\right)$. Hence, $\mathcal{V}_{\varepsilon} \in W^{1,1}\left(0, \infty ; \mu_{\varepsilon}\right)$ so that the identity

$$
\ell\left(u_{\varepsilon}(t),\left|u_{\varepsilon}^{\prime}\right|(t)\right)=\mathcal{V}_{\varepsilon}(t)-\varepsilon \mathcal{V}_{\varepsilon}^{\prime}(t) \quad \text { a.e. in }(0, \infty)
$$

yields, by the integration by parts formula (2.32), that

$$
\mathcal{I}_{\varepsilon}\left[u_{\varepsilon}\right]=\int_{0}^{\infty} \ell\left(u_{\varepsilon},\left|u_{\varepsilon}^{\prime}\right|\right) \mathrm{d} \mu_{\varepsilon}=\int_{0}^{\infty}\left(\mathcal{V}_{\varepsilon}(t)-\varepsilon \mathcal{V}_{\varepsilon}^{\prime}(t)\right) \mathrm{d} \mu_{\varepsilon}(t)=\mathcal{V}_{\varepsilon}(0) .
$$

A similar argument leads to (3.23). 


\section{The value function and its properties}

As we mentioned in the Introduction, Problem 3.1 can be interpreted in the framework of optimal control theory, as the simplest infinite-horizon problem, cf. [BCD97, Chap. III]. In this connection, the associated value function $V_{\varepsilon}: X \rightarrow[0, \infty]$

$$
V_{\varepsilon}(x):=\inf _{u \in \mathscr{C}_{\varepsilon}(x)} \mathcal{I}_{\varepsilon}[u]=\inf _{u \in \mathscr{C}_{\varepsilon}(x)} \int_{0}^{\infty} \ell_{\varepsilon}\left(u,\left|u^{\prime}\right|\right) \mathrm{d} \mu_{\varepsilon}, \quad x \in X,
$$

will play a crucial role. As usual, we will always suppose that $\phi$ satisfies the standard LSCC Property 2.5 and $\frac{1}{16 \varepsilon}>\mathrm{B}$; we also set $\mathrm{D}\left(V_{\varepsilon}\right):=\left\{x \in X: V_{\varepsilon}(x)<\infty\right\}$.

It will also be useful to deal with the corresponding functional associated with the finite-horizon functional from (3.6), namely:

$$
V_{\varepsilon, T}(x):=\inf _{u \in \mathscr{C}_{\varepsilon}, T} \mathcal{I}_{\varepsilon}[u]=\inf _{u \in \mathscr{C}_{\varepsilon, T}(x)} \int_{0}^{T} \ell_{\varepsilon}\left(u,\left|u^{\prime}\right|\right) \mathrm{d} \mu_{\varepsilon}+\mathrm{e}^{-T / \varepsilon} \phi(u(T)), \quad x \in X,
$$

In this section, we first address some general properties of $V_{\varepsilon}$. Then, we use the Dynamic Programming Principle (cf. Proposition 4.2 below) to derive a fundamental equation satisfied by $V_{\varepsilon}$ evaluated along any minimizer $u$ for (4.1), cf. (4.11) below. Then, with the aid of Theorem 4.6 ahead, we will read from (4.11) that WED-minimizers are curves of maximal slope of the value function $V_{\varepsilon}$, in a suitable sense (cf. Corollary 4.7).

Our first result guarantees that the functional $V_{\varepsilon}$ is quadratically bounded from below and lower semicontinuous with respect to the $\sigma$-topology, at least on bounded subsets of $X$.

Lemma 4.1. Let us suppose that $\phi$ satisfies the standard LSCC Property 2.5 and that $\frac{1}{16 \varepsilon} \geq \mathrm{B}$. Then the infimum in (4.1) is attained for every $x \in \mathrm{D}\left(V_{\varepsilon}\right)$ and $V_{\varepsilon}$ itself satisfies the standard $L S C C$ Property 2.5; in particular, $V_{\varepsilon}$ is sequential $\sigma$-lower semicontinuous on $\mathrm{d}$-bounded sets of $X$, and

$$
\phi(x) \geq V_{\varepsilon}(x) \geq-\mathrm{Q}(x)=-\mathrm{A}-\mathrm{Bd}^{2}\left(x, u_{*}\right) \quad \text { for every } x \in X .
$$

Proof. The first two statements are immediate consequences of Theorem 3.2 and Corollary 3.5. Estimate (4.3) follows from (3.7), the representation formula (3.11) for $\mathcal{I}_{\varepsilon}$ and the positivity of the first two integral terms in (3.11), cf. also (3.13).

4.1. The Dynamic Programming Principle and its consequences. Interpreting the WED minimum problem (4.1) in the light of the theory of optimal control provides the following key result.

Proposition 4.2 (Dynamic Programming Principle). If $\phi$ satisfies the standard LSCC Property 2.5, $\frac{1}{16 \varepsilon} \geq \mathrm{B}$, and $x \in \mathrm{D}\left(V_{\varepsilon}\right)$ then

$$
V_{\varepsilon}(x)=\min _{u \in \mathscr{C}_{\varepsilon}(x)}\left(\int_{0}^{T} \ell_{\varepsilon}\left(u,\left|u^{\prime}\right|\right) \mathrm{d} \mu_{\varepsilon}+V_{\varepsilon}(u(T)) \mathrm{e}^{-T / \varepsilon}\right) \quad \text { for every } T>0 .
$$

In particular, every $u_{\varepsilon} \in \mathcal{M}_{\varepsilon}(x)$ is a minimizer for the minimum problem on the right-hand side of (4.4), it satisfies

$$
V_{\varepsilon}(x)=\int_{0}^{T} \ell_{\varepsilon}\left(u_{\varepsilon},\left|u_{\varepsilon}^{\prime}\right|\right) \mathrm{d} \mu_{\varepsilon}+V_{\varepsilon}\left(u_{\varepsilon}(T)\right) \mathrm{e}^{-T / \varepsilon} \quad \text { for all } T>0,
$$

and for every $T>0$ the curve $w_{\varepsilon, T}(t):=u_{\varepsilon}(t+T)$ fulfills

$$
w_{\varepsilon, T} \in \mathcal{M}_{\varepsilon}\left(u_{\varepsilon}(T)\right) .
$$

Proof. Formula (4.4) can be proved arguing along the very same lines as in the proof of [BCD97, Prop. 2.5, Chap. III].

We now prove (4.5). First of all we show that, if $u \in \mathscr{C}_{\varepsilon}(x)$ and $T>0$, then

$$
V_{\varepsilon}(x) \leq \int_{0}^{T} \ell_{\varepsilon}\left(u,\left|u^{\prime}\right|\right) \mathrm{d} \mu_{\varepsilon}+V_{\varepsilon}(u(T)) \mathrm{e}^{-T / \varepsilon} .
$$


It is not restrictive to assume that $V_{\varepsilon}(u(T))<\infty$ : we can then choose $w \in \mathcal{M}_{\varepsilon}(u(T))$ so that

$$
V_{\varepsilon}(u(T))=\int_{0}^{\infty} \ell_{\varepsilon}\left(w,\left|w^{\prime}\right|\right) \mathrm{d} \mu_{\varepsilon}=\mathrm{e}^{T / \varepsilon} \int_{T}^{\infty} \ell_{\varepsilon}\left(w(t-T),\left|w^{\prime}\right|(t-T)\right) \mathrm{d} \mu_{\varepsilon}(t),
$$

and we consider the new curve $v \in \mathscr{C}_{\varepsilon}(x)$ defined by

$$
v(t):= \begin{cases}u(t) & \text { if } t \in[0, T] \\ w(t-T) & \text { if } t \geq T\end{cases}
$$

By the very definition of the value function we have

$$
V_{\varepsilon}(x) \leq \mathcal{I}_{\varepsilon}[v]=\int_{0}^{T} \ell_{\varepsilon}\left(u,\left|u^{\prime}\right|\right) \mathrm{d} \mu_{\varepsilon}+\int_{T}^{\infty} \ell_{\varepsilon}\left(w(t-T),\left|w^{\prime}\right|(t-T)\right) \mathrm{d} \mu_{\varepsilon}(t)
$$

which yields (4.7) thanks to (4.8).

On the other hand, choosing $u_{\varepsilon} \in \mathcal{M}_{\varepsilon}(x)$ and defining $w_{\varepsilon, T}(t):=u_{\varepsilon}(t+T)$, since $w_{\varepsilon, T} \in$ $\mathscr{C}_{\varepsilon}\left(u_{\varepsilon}(T)\right)$ we get

$$
\begin{aligned}
V_{\varepsilon}(x) & =\int_{0}^{T} \ell_{\varepsilon}\left(u_{\varepsilon},\left|u_{\varepsilon}^{\prime}\right|\right) \mathrm{d} \mu_{\varepsilon}+\mathrm{e}^{-T / \varepsilon} \int_{0}^{\infty} \ell_{\varepsilon}\left(u(t+T),\left|u^{\prime}\right|(t+T)\right) \mathrm{d} \mu_{\varepsilon}(t) \\
& =\int_{0}^{T} \ell_{\varepsilon}\left(u_{\varepsilon},\left|u_{\varepsilon}^{\prime}\right|\right) \mathrm{d} \mu_{\varepsilon}+\mathrm{e}^{-T / \varepsilon} \mathcal{I}\left[w_{\varepsilon, T}\right] \geq \int_{0}^{T} \ell_{\varepsilon}\left(u_{\varepsilon},\left|u_{\varepsilon}^{\prime}\right|\right) \mathrm{d} \mu_{\varepsilon}+\mathrm{e}^{-T / \varepsilon} V_{\varepsilon}\left(u_{\varepsilon}(T)\right) ;
\end{aligned}
$$

by (4.7) the previous (4.9) is in fact an equality, which shows that $u_{\varepsilon}$ satisfies (4.5), is a minimizer of (4.4) and satisfies $\mathcal{I}\left[w_{\varepsilon, T}\right]=V_{\varepsilon}\left(u_{\varepsilon}(T)\right)$, which yields $(4.6)$.

Relation (4.5) has a simple but important differential version, which will be the starting point for our asymptotic analysis when $\varepsilon \downarrow 0$. In order to highlight its structure, we introduce the function

$$
G_{\varepsilon}(x):= \begin{cases}\sqrt{2 \frac{\phi(x)-V_{\varepsilon}(x)}{\varepsilon}} & \text { if } x \in D\left(V_{\varepsilon}\right), \\ \infty & \text { otherwise }\end{cases}
$$

which, in the next sections, will be shown to suitably approximate the (relaxed) slope of $\phi$.

Proposition 4.3 (Fundamental identity). Let us suppose that $\phi$ satisfies the standard LSCC Property 2.5 and $\frac{1}{16 \varepsilon} \geq \mathrm{B}$. If $x \in \mathrm{D}\left(V_{\varepsilon}\right)$ and $u_{\varepsilon} \in \mathcal{M}_{\varepsilon}(x)$, the map $t \mapsto V_{\varepsilon}\left(u_{\varepsilon}(t)\right)$ is absolutely continuous, and it fulfills

$$
\begin{aligned}
-\frac{\mathrm{d}}{\mathrm{d} t} V_{\varepsilon}\left(u_{\varepsilon}(t)\right) & =\frac{1}{2}\left|u_{\varepsilon}^{\prime}\right|^{2}(t)+\frac{1}{\varepsilon} \phi\left(u_{\varepsilon}(t)\right)-\frac{1}{\varepsilon} V_{\varepsilon}\left(u_{\varepsilon}(t)\right) \\
& =\frac{1}{2}\left|u_{\varepsilon}^{\prime}\right|^{2}(t)+\frac{1}{2} G_{\varepsilon}^{2}\left(u_{\varepsilon}(t)\right) \quad \text { for a.a. } t \in(0, \infty), \\
V_{\varepsilon}\left(u_{\varepsilon}(t)\right)= & \phi\left(u_{\varepsilon}(t)\right)-\frac{\varepsilon}{2}\left|u_{\varepsilon}^{\prime}\right|^{2}(t) \quad \text { for a.a. } t \in(0, \infty) .
\end{aligned}
$$

Proof. It follows from the Dynamic Programming Principle (4.4) that for any $u_{\varepsilon} \in \mathcal{M}_{\varepsilon}(\bar{u})$ and for all $0 \leq s \leq t$ there holds

$$
\mathrm{e}^{-s / \varepsilon} V_{\varepsilon}\left(u_{\varepsilon}(s)\right)-\mathrm{e}^{-t / \varepsilon} V_{\varepsilon}\left(u_{\varepsilon}(t)\right)=\int_{s}^{t}\left(\frac{1}{2}\left|u_{\varepsilon}^{\prime}\right|(r)^{2}+\frac{1}{\varepsilon} \phi\left(u_{\varepsilon}(r)\right)\right) \mathrm{e}^{-t / \varepsilon} \mathrm{d} r,
$$

which shows that the map $t \mapsto \mathrm{e}^{-t / \varepsilon} V_{\varepsilon}\left(u_{\varepsilon}(t)\right)$ is absolutely continuous. The Lebesgue Theorem then yields that

$$
\mathrm{e}^{-t / \varepsilon}\left(\frac{1}{\varepsilon} V_{\varepsilon}\left(u_{\varepsilon}(t)\right)-\frac{\mathrm{d}}{\mathrm{d} t} V_{\varepsilon}\left(u_{\varepsilon}(t)\right)\right)=\mathrm{e}^{-t / \varepsilon}\left(\frac{1}{2}\left|u_{\varepsilon}^{\prime}\right|(t)^{2}+\frac{1}{\varepsilon} \phi\left(u_{\varepsilon}(t)\right)\right) \quad \text { for a.a. } t \in(0, \infty),
$$

and therefore (4.11).

In order to get (4.12) we denote by $\mathcal{V}_{\varepsilon}$ the absolutely continuous representative of the function $t \mapsto \phi\left(u_{\varepsilon}(t)\right)-\frac{\varepsilon}{2}\left|u_{\varepsilon}^{\prime}\right|^{2}(t)$ on $(0, \infty)$. The inner variation equation (3.20) gives that the distributional 
derivative of $\mathcal{V}_{\varepsilon}$ fulfills $\frac{\mathrm{d}}{\mathrm{d} t} \mathcal{V}_{\varepsilon}(t)=-\left|u_{\varepsilon}^{\prime}\right|^{2}(t) \in L^{1}\left(0, \infty ; \mu_{\varepsilon}\right)$. Hence, $\mathcal{V}_{\varepsilon} \in W^{1,1}\left(0, \infty ; \mu_{\varepsilon}\right)$ so that the identity

$$
\ell\left(u_{\varepsilon}(t),\left|u_{\varepsilon}^{\prime}\right|(t)\right)=\mathcal{V}_{\varepsilon}(t)-\varepsilon \mathcal{V}_{\varepsilon}^{\prime}(t) \quad \text { a.e. in }(0, \infty)
$$

yields by the integration by parts formula (2.38) that

$$
V_{\varepsilon}\left(u_{\varepsilon}(t)\right)=\int_{0}^{\infty} \ell\left(u_{\varepsilon}(t+\tau),\left|u_{\varepsilon}^{\prime}\right|(t+\tau)\right) \mathrm{d} \mu_{\varepsilon}(\tau)=\int_{0}^{\infty}\left(\mathcal{V}_{\varepsilon}(t+\tau)-\varepsilon \mathcal{V}_{\varepsilon}^{\prime}(t+\tau)\right) \mathrm{d} \mu_{\varepsilon}(\tau)=\mathcal{V}_{\varepsilon}(t)
$$

for every $t \geq 0$.

As a consequence of (4.12), we deduce some integral estimates on $\phi\left(u_{\varepsilon}\right)$ and $\left|u_{\varepsilon}^{\prime}\right|$ uniformly with respect to $\varepsilon>0$.

Corollary 4.4. Let us suppose that $\phi$ satisfies the standard LSCC Property 2.5 and $\frac{1}{16 \varepsilon}>$ B. Then, every $u_{\varepsilon} \in \mathcal{M}_{\varepsilon}(\bar{u})$ fulfills the following energy identity

$$
V_{\varepsilon}\left(u_{\varepsilon}(t)\right)+\int_{s}^{t}\left|u_{\varepsilon}^{\prime}\right|^{2}(r) \mathrm{d} r=V_{\varepsilon}\left(u_{\varepsilon}(s)\right) \quad \text { for all } 0 \leq s \leq t<\infty .
$$

Furthermore the following estimates hold

$$
\begin{aligned}
& \int_{0}^{T}\left|u_{\varepsilon}^{\prime}\right|^{2}(t) \mathrm{d} t \leq 2\left(V_{\varepsilon}(\bar{u})+\mathrm{Q}(\bar{u})\right) \mathrm{e}^{2 \mathrm{~B} T}, \\
& \int_{0}^{T} \phi\left(u_{\varepsilon}(t)\right) \mathrm{d} t \leq T \phi(\bar{u})+\varepsilon\left(V_{\varepsilon}(\bar{u})+\mathrm{Q}(\bar{u})\right) \mathrm{e}^{2 \mathrm{~B} T} .
\end{aligned}
$$

for all $T \geq 0$ and all $\varepsilon>0$.

Proof. Combining (4.12) and the metric inner variation equation (3.20) we obtain

$$
\frac{\mathrm{d}}{\mathrm{d} t} V_{\varepsilon}\left(u_{\varepsilon}(t)\right)+\left|u_{\varepsilon}^{\prime}\right|^{2}(t)=0 \text { for a.a. } t \in(0, \infty),
$$

yielding (4.14).

We now introduce the function

$$
W_{\varepsilon}(x):=V_{\varepsilon}(x)+2 \mathrm{Q}(x), \quad \text { satisfying } \quad 0 \leq \max \left(V_{\varepsilon}(x), \mathrm{Q}(x)\right) \leq W_{\varepsilon}(x) \text { for every } x \in X,
$$

(with Q from $(2.19 \mathrm{c})$ ), and we set $w_{\varepsilon}(t):=W_{\varepsilon}\left(u_{\varepsilon}(t)\right)$. Then, $w_{\varepsilon}$ is an absolutely continuous function satisfying the differential inequality

$$
\frac{\mathrm{d}}{\mathrm{d} t} w_{\varepsilon} \leq-\left|u_{\varepsilon}^{\prime}\right|^{2}+4 \mathrm{Bd}\left(u_{\varepsilon}, u_{*}\right)\left|u_{\varepsilon}^{\prime}\right| \leq 4 \mathrm{~B}^{2} \mathrm{~d}^{2}\left(u_{\varepsilon}, u_{*}\right) \leq 4 \mathrm{BQ}\left(u_{\varepsilon}\right) \leq 2 \mathrm{~B} w_{\varepsilon}
$$

where we have used (4.16) and, for the second inequality, the elementary estimate $x y \leq x^{2}+\frac{1}{4} y^{2}$, so that

$$
\mathrm{Q}\left(u_{\varepsilon}(t)\right) \leq w_{\varepsilon}(t) \leq w_{\varepsilon}(0) \mathrm{e}^{2 \mathrm{~B} t}=\left(V_{\varepsilon}(\bar{u})+2 \mathrm{Q}(\bar{u})\right) \mathrm{e}^{2 \mathrm{~B} t} .
$$

We then have that

$$
\int_{0}^{T}\left|u_{\varepsilon}^{\prime}\right|^{2} \mathrm{~d} t=V_{\varepsilon}(\bar{u})-V_{\varepsilon}\left(u_{\varepsilon}(T)\right) \leq V_{\varepsilon}(\bar{u})+\mathrm{Q}\left(u_{\varepsilon}(T)\right) \leq 2\left(V_{\varepsilon}(\bar{u})+\mathrm{Q}(\bar{u})\right) \mathrm{e}^{2 \mathrm{~B} t},
$$

where the first estimate follows from (4.3) and the last one from (4.19). This yields (4.15a).

It follows from (4.3) and (4.12) that

$$
\phi\left(u_{\varepsilon}(t)\right) \leq \phi(\bar{u})+\frac{\varepsilon}{2}\left|u_{\varepsilon}^{\prime}\right|^{2}(t) \quad \text { for all } t \in[0, \infty) .
$$

Hence, a further integration over $(0, T)$ gives $(4.15 \mathrm{~b})$. 
4.2. Gradient flow of the value function. In this section we will show that any minimizer $u_{\varepsilon}$ of the WED functional $\mathcal{I}_{\varepsilon}$ of Problem 3.1 is a curve of maximal slope for the value function $V_{\varepsilon}$ with respect to its $L^{1}$-moderated upper gradient

$$
G_{\varepsilon}(x):= \begin{cases}\sqrt{2 \frac{\phi(x)-V_{\varepsilon}(x)}{\varepsilon}} & \text { if } x \in D\left(V_{\varepsilon}\right), \\ \infty & \text { otherwise. }\end{cases}
$$

In the forthcoming Theorem 4.6 we will show that $G_{\varepsilon}$ is an $L^{1}$-moderated upper gradient (in the sense specified in (2.5)) of $V_{\varepsilon}$. We also refer to Appendix A ahead for further results in this connection. We first state a useful Lemma.

Lemma 4.5. Let $u \in \mathrm{AC}^{2}([0, T] ; X)$ with $\phi \circ u \in L^{1}(0, T)$. Then for every $0 \leq r<s<T$ we have

$$
\begin{aligned}
\mathrm{e}^{-r / \varepsilon} V_{\varepsilon}(u(r))-\mathrm{e}^{-s / \varepsilon} V_{\varepsilon}(u(s)) & \leq \int_{r}^{s}\left(\frac{1}{2}\left|u^{\prime}\right|^{2}+\frac{1}{\varepsilon} \phi(u)\right) \mathrm{e}^{-t / \varepsilon} \mathrm{d} t, \\
\mathrm{e}^{s / \varepsilon} V_{\varepsilon}(u(s))-\mathrm{e}^{r / \varepsilon} V_{\varepsilon}(u(r)) & \leq \int_{r}^{s}\left(\frac{1}{2}\left|u^{\prime}\right|^{2}+\frac{1}{\varepsilon} \phi(u)\right) \mathrm{e}^{t / \varepsilon} \mathrm{d} t .
\end{aligned}
$$

Proof. Let us fix $r<s \in[0, T]$, let us choose $w \in \mathcal{M}_{\varepsilon}(u(s))$ and let us consider the curve

$$
v(t):= \begin{cases}u(r+t) & \text { if } 0 \leq t \leq s-r, \\ w(t-(s-r)) & \text { if } t \geq s-r\end{cases}
$$

so that

$$
V_{\varepsilon}(u(r)) \leq \mathcal{I}_{\varepsilon}[v]=\int_{r}^{s} \ell\left(u(t),\left|u^{\prime}\right|(t)\right) \frac{\mathrm{e}^{-(t-r) / \varepsilon}}{\varepsilon} \mathrm{d} t+\mathrm{e}^{-(s-r) / \varepsilon} V_{\varepsilon}(u(s))
$$

Multiplying the previous inequality by $\mathrm{e}^{-r / \varepsilon}$ we get (4.21). Applying the same argument inverting the order of time we infer (4.22).

Theorem 4.6. Under the standard LSCC Property 2.5, for every $\varepsilon>0$ and for every $u \in$ $\operatorname{AC}^{2}([0, T] ; X)$ such that $V_{\varepsilon} \circ u \in L^{1}(0, T)$ and $G_{\varepsilon} \circ u \in L^{2}(0, T)$ we have that

$$
\begin{aligned}
& \text { the map } t \mapsto V_{\varepsilon}(u(t)) \quad \text { is absolutely continuous on }[0, T] ; \\
& \left|\frac{\mathrm{d}}{\mathrm{d} t} V_{\varepsilon}(u(t))\right| \leq \frac{1}{2} G_{\varepsilon}^{2}(u(t))+\frac{1}{2}\left|u^{\prime}\right|^{2}(t) \quad \text { for a.a. } t \in(0, T) .
\end{aligned}
$$

In particular $G_{\varepsilon}$ is an $L^{1}$-moderated upper gradient of $V_{\varepsilon}$.

Proof. Let $u \in \mathrm{AC}^{2}([0, T] ; X)$ be fixed according to the assumptions of the theorem. Since $\phi=$ $\frac{\varepsilon}{2} G_{\varepsilon}^{2}+V_{\varepsilon}$ by the definition of $G_{\varepsilon}$, we get $\phi \circ u \in L^{1}(0, T)$. Setting $z(t):=\mathrm{e}^{-t / \varepsilon} V_{\varepsilon}(u(t)), H_{\varepsilon}(t):=$ $\int_{0}^{t}\left(\frac{1}{2}\left|u^{\prime}\right|^{2}+\frac{1}{\varepsilon} \phi_{+}(u)\right) \mathrm{d} r,(4.21)$ yields

$$
z(r)-z(s) \leq H_{\varepsilon}(s)-H_{\varepsilon}(r) \quad \text { if } 0 \leq r \leq s \leq T .
$$

It follows that the map $t \mapsto z(t)-H_{\varepsilon}(t)$ is nondecreasing; since it is integrable, $z$ and $V_{\varepsilon} \circ u$ are locally bounded in $(0, T)$ : let us set $S(I):=\frac{1}{\varepsilon} \sup _{I}\left|V_{\varepsilon} \circ u\right|$ where $I \subset(0, T)$ is a compact interval. Multiplying inequality $(4.21)$ by $\mathrm{e}^{r / \varepsilon}$ we obtain

$$
V_{\varepsilon}(u(r))-V_{\varepsilon}(u(s)) \leq\left(\mathrm{e}^{-(s-r) / \varepsilon}-1\right) V_{\varepsilon}(u(s))+H_{\varepsilon}(s)-H_{\varepsilon}(r) .
$$

We then estimate the first term on the right-hand side by resorting to the elementary inequality $0 \leq 1-\mathrm{e}^{-x} \leq x$ for $x \geq 0$, which yields

$$
V_{\varepsilon}(u(r))-V_{\varepsilon}(u(s)) \leq S(I)(s-r)+H_{\varepsilon}(s)-H_{\varepsilon}(r) \quad r \leq s, r, s \in I .
$$

Multiplying (4.22) by $\mathrm{e}^{-s / \varepsilon}$ and arguing in the very same way we obtain (4.25) with the order of $r$ and $s$ interchanged. We thus get

$$
\left|V_{\varepsilon}(u(r))-V_{\varepsilon}(u(s))\right| \leq S(I)|s-r|+|H(s)-H(r)| \quad r, s \in I,
$$

which shows that $V_{\varepsilon} \circ u$ is locally absolutely continuous. 
Let us fix now a time $r \in(0, T)$ which is a differentiability point for $V_{\varepsilon} \circ u$ and a Lebesgue point for the integrand in (4.22). Dividing this inequality by $s-r$ and passing to the limit as $s \downarrow 0$ we obtain

which shows

$$
\mathrm{e}^{r / \varepsilon}\left(V_{\varepsilon} \circ u\right)^{\prime}(r)+\frac{1}{\varepsilon} \mathrm{e}^{r / \varepsilon} V_{\varepsilon}(u(r)) \leq\left(\frac{1}{2}\left|u^{\prime}\right|^{2}(r)+\frac{1}{\varepsilon} \phi(u(r))\right) \mathrm{e}^{r / \varepsilon}
$$

$$
\left(V_{\varepsilon} \circ u\right)^{\prime}(r) \leq \frac{1}{2}\left|u^{\prime}\right|^{2}(r)+\frac{1}{2} G_{\varepsilon}\left(u_{\varepsilon}(r)\right) .
$$

A similar argument applied to (4.21) yields the opposite inequality, thus leading to (4.24) and the absolute continuity of $V_{\varepsilon} \circ u$ in $(0, T)$.

Since $V_{\varepsilon} \circ u$ is also lower semicontinuous, passing to the limit as $s \downarrow 0$ in (4.22) written for $r=0$ yields the continuity of $V_{\varepsilon} \circ u$ at $r=0$. A similar argument applied to (4.21) at $s=T$ yields the continuity of $V_{\varepsilon} \circ u$ at $T$.

We conclude the proof that $G_{\varepsilon}$ is an $L^{1}$-moderated upper gradient by integrating (4.24) from 0 to $T$ and applying Corollary 2.3.

Corollary 4.7. Every $u_{\varepsilon} \in \mathcal{M}_{\varepsilon}(\bar{u})$ is a curve of maximal slope for $V_{\varepsilon}$ with respect to the $\left(L^{1}\right.$ moderated) upper gradient $G_{\varepsilon}$.

\section{Passage to the limit as $\varepsilon \rightarrow 0$ And Proof of Theorem 3.6}

The proof of Theorem 3.6 is carried out in Section 5.3 and relies on a series of intermediate results on the asymptotic properties of the functionals $\left(V_{\varepsilon}\right)_{\varepsilon}$ as $\varepsilon \downarrow 0$, proved in Sec. 5.1. As usual, we will always assume that the functional $\phi$ satisfies the standard LSCC Property 2.5 .

\subsection{Comparison and asymptotic properties of the functionals $\left(V_{\varepsilon}\right)_{\varepsilon}$ as $\varepsilon \downarrow 0$.}

Lemma 5.1. Let us suppose that the standard LSCC Property 2.5 holds. Then,

(1) For every $\bar{u} \in X$ the map $\varepsilon \mapsto V_{\varepsilon}(\bar{u})$ is non increasing, i.e.

$$
V_{\varepsilon_{1}}(\bar{u}) \leq V_{\varepsilon_{0}}(\bar{u}) \quad \text { for all } \bar{u} \in X \quad \text { and all } \varepsilon_{1} \geq \varepsilon_{0} ;
$$

(2) For every $\bar{u} \in X$ there holds

$$
V_{\varepsilon}(\bar{u}) \uparrow \phi(\bar{u}) \quad \text { as } \varepsilon \downarrow 0 ;
$$

(3) Every family $\left(\bar{u}_{\varepsilon}\right)_{\varepsilon>0} \subset X$ satisfies the $\Gamma$-liminf inequality

$$
\bar{u}_{\varepsilon} \stackrel{\sigma}{\rightarrow} \bar{u}, \quad \limsup _{\varepsilon \downarrow 0} \mathrm{~d}\left(\bar{u}_{\varepsilon}, \bar{u}\right)<\infty \quad \Rightarrow \quad \phi(\bar{u}) \leq \liminf _{\varepsilon \downarrow 0} V_{\varepsilon}\left(\bar{u}_{\varepsilon}\right) .
$$

Proof. The monotonicity property (5.1) is a consequence of the equivalent representation of $V_{\varepsilon}$ as

$$
V_{\varepsilon}(\bar{u})=\min _{u \in \mathscr{C}_{\varepsilon}(\bar{u})} \int_{0}^{\infty}\left(\frac{1}{2 \varepsilon^{2}}\left|u_{\varepsilon}^{\prime}\right|^{2}(t)+\phi(u(t))\right) \mathrm{e}^{-t} \mathrm{~d} t .
$$

Convergence (5.2) immediately follows from (5.1) and (5.3). In order to prove the latter property, it is not restrictive to assume that $V_{\varepsilon}\left(\bar{u}_{\varepsilon}\right) \leq V<\infty$ for sufficiently small $\varepsilon$ : then, Problem 3.1 is feasible, $\mathcal{M}_{\varepsilon}\left(\bar{u}_{\varepsilon}\right) \neq \emptyset$ by Thm. 3.2 , and we can choose $u_{\varepsilon} \in \mathcal{M}_{\varepsilon}\left(\bar{u}_{\varepsilon}\right)$ as in (3.11) we set $\psi_{\varepsilon}(t):=\phi\left(u_{\varepsilon}(t)\right)+\frac{1}{16 \varepsilon}\left(\int_{0}^{t}\left|u_{\varepsilon}^{\prime}\right| \mathrm{d} r\right)^{2}+\mathrm{Q}$, where $\mathrm{Q}>\lim \sup _{\varepsilon \downarrow 0} \mathrm{Q}\left(\bar{u}_{\varepsilon}\right)$, so that

$$
V_{\varepsilon}\left(\bar{u}_{\varepsilon}\right) \geq \int_{0}^{\infty} \frac{\mathrm{e}^{-t / \varepsilon}}{\varepsilon} \psi_{\varepsilon}(t) \mathrm{d} t-\mathrm{Q}=\int_{0}^{\infty} \mathrm{e}^{-s} \psi_{\varepsilon}(\varepsilon s) \mathrm{d} s-\mathrm{Q} .
$$

Observe now that the uniform estimate (4.15a) yields

$$
\begin{gathered}
\mathrm{d}\left(u_{\varepsilon}(\varepsilon s), \bar{u}_{\varepsilon}\right) \leq\left(\varepsilon s \int_{0}^{\varepsilon s}\left|u_{\varepsilon}^{\prime}\right|^{2} \mathrm{~d} t\right)^{1 / 2} \leq \sqrt{2 \varepsilon s}\left(V\left(\bar{u}_{\varepsilon}\right)+\mathrm{Q}\left(\bar{u}_{\varepsilon}\right)\right)^{1 / 2} \mathrm{e}^{\mathrm{B} \varepsilon s}, \\
\text { so that } \quad \lim _{\varepsilon \downarrow 0} \mathrm{~d}\left(u_{\varepsilon}(\varepsilon s), \bar{u}_{\varepsilon}\right)=0,
\end{gathered}
$$


so that for every $s>0$

$$
\lim _{\varepsilon \downarrow 0} u_{\varepsilon}(\varepsilon s)=\bar{u} \quad \text { in the } \sigma \text {-topology, } \quad \liminf _{\varepsilon \downarrow 0} \psi_{\varepsilon}(\varepsilon s) \geq \phi(\bar{u})+\mathrm{Q} .
$$

Eventually, an application of Fatou's lemma to (5.5) yields $\liminf \operatorname{i\downarrow }_{\varepsilon \downarrow} V_{\varepsilon}\left(\bar{u}_{\varepsilon}\right) \geq \phi(\bar{u})$.

The next result provides a lower bound of $V_{\varepsilon}$ in terms of the Yosida regularization of $\phi$, defined as

$$
\phi_{t}(x):=\inf _{y \in X}\left(\frac{1}{2 t} \mathrm{~d}^{2}(y, x)+\phi(y)\right) \quad x \in X, t>0 .
$$

Notice that

$$
\phi_{t}(x) \geq-\mathrm{Q}(x) \text { if } \frac{1}{2 t} \geq \mathrm{B},
$$

and $\phi_{t}$ is uniformly bounded from below if $\phi$ is bounded from below. Let us mention in advance that the upcoming (5.9) will be used for establishing a key inequality between the local slope $|\partial \phi|$ and $\lim \sup _{\varepsilon \downarrow 0} G_{\varepsilon}$.

Theorem 5.2. For every $x \in X$ and $T>0$ such that $\frac{1}{4 T} \geq \mathrm{B}$ we have

$$
V_{\varepsilon}(x) \geq \int_{0}^{T} \phi_{t}(x) \mathrm{d} \mu_{\varepsilon}(t)-2 \mathrm{Q}(x) \mathrm{e}^{-T / \varepsilon} \quad \text { for every } T>0 ;
$$

in particular, there holds

$$
V_{\varepsilon}(x) \geq \int_{0}^{\infty} \phi_{t}(x) \mathrm{d} \mu_{\varepsilon}(t) .
$$

Proof. For every $u \in \mathscr{C}_{\varepsilon}(x)$ we introduce the energy functional

$$
\mathrm{E}(t):=\int_{0}^{t}\left|u^{\prime}\right|^{2}(s) \mathrm{d} s .
$$

Formula (2.33) yields

$$
\int_{0}^{T} \frac{\varepsilon}{2}\left|u^{\prime}\right|^{2}(t) \mathrm{d} \mu_{\varepsilon}(t)=\int_{0}^{T} \frac{1}{2} \mathrm{E}(t) \mathrm{d} \mu_{\varepsilon}(t)+\frac{\mathrm{e}^{-T / \varepsilon}}{2} \mathrm{E}(T),
$$

so that

$$
\mathcal{I}[u] \geq \int_{0}^{T}\left(\frac{1}{2} \mathrm{E}(t)+\phi(u(t))\right) \mathrm{d} \mu_{\varepsilon}(t)+\left(\frac{1}{2} \mathrm{E}(T)+V_{\varepsilon}(u(T))\right) \mathrm{e}^{-T / \varepsilon} .
$$

On the other hand

$$
\mathrm{E}(t) \geq \frac{1}{t}\left(\int_{0}^{t}\left|u^{\prime}\right|(s) \mathrm{d} s\right)^{2} \geq \frac{\mathrm{d}^{2}(u(t), x)}{t}, \quad V_{\varepsilon}(u(T)) \geq-2 \mathrm{Q}(x)-2 \mathrm{Bd}^{2}(u(T), x)
$$

so that, taking into account that $B \leq \frac{1}{4 T}$, we find

$$
\mathcal{I}[u] \geq \int_{0}^{T}\left(\frac{1}{2 t} \mathrm{~d}^{2}(u(t), x)+\phi(u(t))\right) \mathrm{d} \mu_{\varepsilon}(t)-2 \mathrm{Q}(x) \mathrm{e}^{-T / \varepsilon} .
$$

(5.10) immediately follows from (5.9).

5.2. The WED slope and its relaxation. Let us now introduce the functional

$$
\left|\partial_{\mathrm{w}} \phi\right|(x):=\limsup _{\varepsilon \downarrow 0} G_{\varepsilon}(x)=\limsup _{\varepsilon \downarrow 0} \sqrt{2 \frac{\phi(x)-V_{\varepsilon}(x)}{\varepsilon}} \text { for all } x \in \mathrm{D}(\phi),
$$

which shall be referred to as the WED slope of $\phi$; as usual we set $\left|\partial_{\mathrm{w}} \phi\right|(x)=\infty$ if $x \notin \mathrm{D}(\phi)$. We also introduce its lower semicontinuous relaxation with respect to the $\sigma$-topology, along d-bounded sequences with bounded energy, viz.

$$
\left|\partial_{\mathrm{w}}^{-} \phi\right|(x):=\inf \left\{\liminf _{n \uparrow \infty}\left|\partial_{\mathrm{w}} \phi\right|\left(x_{n}\right): x_{n} \stackrel{\sigma}{\rightarrow} x, \sup _{n}\left(\mathrm{~d}\left(x_{n}, x\right), \phi\left(x_{n}\right)\right)<\infty\right\} .
$$

We shall refer to $\left|\partial_{\mathrm{w}}^{-} \phi\right|$ as the relaxed WED slope of $\phi$.

In Proposition 5.3 below we prove that $\left|\partial_{\mathrm{w}} \phi\right|$ is dominated by the local slope of $\phi$. 
Proposition 5.3. If $\phi$ satisfies (2.19c). then

$$
\left|\partial_{\mathrm{w}} \phi\right|(x) \leq|\partial \phi|(x) \quad \text { for every } x \in \mathrm{D}(\phi) .
$$

Proof. We recall the duality formula for the local slope [AGS08, Lemma 3.1.5]

$$
\frac{1}{2}|\partial \phi|^{2}(x)=\limsup _{t \downarrow 0} \frac{\phi(x)-\phi_{t}(x)}{t} \quad \text { for every } x \in \mathrm{D}(\phi) .
$$

It is not restrictive to suppose $|\partial \phi|(x)<\infty$ so that by (5.8) there exists a constant $C \geq|\partial \phi|(x)$ such that

$$
0 \leq \frac{\phi(x)-\phi_{t}(x)}{t} \leq C \quad \text { if } 0<t<\frac{1}{2 \mathrm{~B}} .
$$

Choosing $T$ so that $0<T \leq \frac{1}{4 \mathrm{~B}}$, by $(5.9)$ we get

$$
\begin{aligned}
\frac{\phi(x)-V_{\varepsilon}(x)}{\varepsilon} & \leq \int_{0}^{T} \frac{\phi(x)-\phi_{t}(x)}{\varepsilon} \mathrm{d} \mu_{\varepsilon}(t)+\frac{\mathrm{e}^{-T / \varepsilon}}{\varepsilon}(\phi(x)+2 \mathrm{Q}(x)) \\
& =\int_{0}^{T / \varepsilon} \frac{\phi(x)-\phi_{\varepsilon t}(x)}{\varepsilon t} t \mathrm{e}^{-t} \mathrm{~d} t+\frac{\mathrm{e}^{-T / \varepsilon}}{\varepsilon}(\phi(x)+2 \mathrm{Q}(x)) \\
& \leq \int_{0}^{\infty}\left(C \wedge \frac{\phi(x)-\phi_{\varepsilon t}(x)}{\varepsilon t}\right) t \mathrm{e}^{-t} \mathrm{~d} t+\frac{\mathrm{e}^{-T / \varepsilon}}{\varepsilon}(\phi(x)+2 \mathrm{Q}(x)),
\end{aligned}
$$

where the last inequality follows from (5.20). Since the last integrand is uniformly bounded, Fatou's Lemma yields

$$
\begin{aligned}
\frac{1}{2}\left|\partial_{\mathrm{w}} \phi\right|^{2}(x) & \leq \int_{0}^{\infty} \limsup _{\varepsilon \downarrow 0}\left(C \wedge\left(\frac{\phi(x)-\phi_{\varepsilon t}(x)}{\varepsilon t}\right)\right) t \mathrm{e}^{-t} \mathrm{~d} t \\
& \leq \int_{0}^{\infty} \frac{1}{2}|\partial \phi|^{2}(x) t \mathrm{e}^{-t} \mathrm{~d} t=\frac{1}{2}|\partial \phi|^{2}(x) .
\end{aligned}
$$

With our next result we provide the converse estimate of (5.18), cf. (5.23), in terms of the relaxed slopes $\left|\partial^{-} \phi\right|$ and $\left|\partial_{\mathrm{w}}^{-} \phi\right|$. Indeed, we shall derive it from estimate (5.22), which will play a key role in the proof of Theorem 3.6. It involves $\left|\partial^{-} \phi\right|$ and the lower semicontinuous relaxation of $G_{\varepsilon}$ itself, with respect to the $\sigma$-topology, along d-bounded sequences with bounded energy, and along vanishing sequences $\left(\varepsilon_{n}\right)_{n}$, i.e.

$$
\mathscr{G}^{-}(x):=\inf \left\{\liminf _{n \uparrow \infty} G_{\varepsilon_{n}}\left(x_{n}\right): \varepsilon_{n} \downarrow 0, x_{n} \stackrel{\sigma}{\rightarrow} x, \sup _{n}\left(\mathrm{~d}\left(x_{n}, x\right), \phi\left(x_{n}\right)\right)<\infty\right\} .
$$

Proposition 5.4. Assume Property 2.5. Then, for every $\bar{u} \in \mathrm{D}(\phi)$ there holds

$$
\begin{aligned}
& \mathscr{G}^{-}(\bar{u}) \geq\left|\partial^{-} \phi\right|(\bar{u}), \\
& \left|\partial_{\mathrm{w}}^{-} \phi\right|(\bar{u}) \geq\left|\partial^{-} \phi\right|(\bar{u}) .
\end{aligned}
$$

Proof. Let us fix a vanishing sequence $\left(\varepsilon_{n}\right)_{n}$ and a sequence $\bar{u}_{n} \stackrel{\sigma}{\rightarrow} \bar{u}$ with $\sup _{n}\left(\mathrm{~d}\left(\bar{u}_{n}, \bar{u}\right), \phi\left(\bar{u}_{n}\right)\right) \leq$ $C<\infty$. From the definition of $V_{\varepsilon_{n}}$ we have

$$
\frac{1}{\varepsilon_{n}}\left(\phi\left(\bar{u}_{n}\right)-V_{\varepsilon_{n}}\left(\bar{u}_{n}\right)\right) \geq \frac{1}{\varepsilon_{n}} \int_{0}^{\infty}\left(\phi\left(\bar{u}_{n}\right)-\phi\left(w_{\varepsilon_{n}}(t)\right)\right) \mathrm{d} \mu_{\varepsilon_{n}}(t)-\frac{1}{\varepsilon_{n}} \int_{0}^{\infty} \frac{\mathrm{e}^{-t / \varepsilon_{n}}}{2}\left|w_{\varepsilon_{n}}^{\prime}\right|^{2}(t) \mathrm{d} t
$$

for every $w_{\varepsilon_{n}} \in \mathscr{C}_{\varepsilon_{n}}\left(\bar{u}_{n}\right)$. In order to show that

$$
\liminf _{n \rightarrow \infty} \frac{1}{\varepsilon_{n}}\left(\phi\left(\bar{u}_{n}\right)-V_{\varepsilon_{n}}\left(\bar{u}_{n}\right)\right) \geq \frac{1}{2}\left|\partial^{-} \phi\right|^{2}(\bar{u}),
$$

we pick $w_{\varepsilon_{n}}$ such that, additionally, it fulfills for every $n \in \mathbb{N}$

$$
\int_{0}^{t}\left(\frac{1}{2}\left|w_{\varepsilon_{n}}^{\prime}\right|^{2}(s)+\frac{1}{2}\left|\partial^{-} \phi\right|^{2}\left(w_{\varepsilon_{n}}(s)\right)\right) \mathrm{d} s+\phi\left(w_{\varepsilon_{n}}(t)\right) \leq \phi\left(w_{\varepsilon_{n}}(0)\right)=\phi\left(\bar{u}_{n}\right) \quad \text { for all } t>0
$$

and such that

$$
\sup _{n \in \mathbb{N}, t \in[0, \infty)}\left(\phi\left(w_{\varepsilon_{n}}(t)\right)+\int_{0}^{t} \mathcal{H}_{\varepsilon_{n}}(s) \mathrm{d} s\right) \leq \phi\left(\bar{u}_{n}\right)
$$


where we have used the place holder $\mathcal{H}_{\varepsilon_{n}}(s):=\frac{1}{2}\left|w_{\varepsilon_{n}}^{\prime}\right|^{2}(s)+\frac{1}{2}\left|\partial^{-} \phi\right|^{2}\left(w_{\varepsilon_{n}}(s)\right)$. In fact it has been shown in [AGS08, Thm. 2.3.1, Lemma 3.2.2] that, under Property 2.5, for every $n \in N$ there exists $w_{\varepsilon_{n}} \in \mathscr{C}_{\varepsilon_{n}}\left(\bar{u}_{n}\right)$ complying with (5.26)-(5.27).

In the following lines, we derive some finer estimates for the sequence $\left(w_{\varepsilon_{n}}\right)_{n}$. Indeed, for almost all $t \in(0, \infty)$

$$
\frac{\mathrm{d}}{\mathrm{d} t} \frac{1}{2} \mathrm{~d}^{2}\left(w_{\varepsilon_{n}}(t), w_{\varepsilon_{n}}(0)\right) \leq \mathrm{d}\left(w_{\varepsilon}(t), w_{\varepsilon_{n}}(0)\right)\left|w_{\varepsilon_{n}}^{\prime}\right|(t) \leq \frac{\delta}{2}\left|w_{\varepsilon_{n}}^{\prime}\right|^{2}(t)+\frac{1}{2 \delta} \mathrm{d}^{2}\left(w_{\varepsilon_{n}}(t), \bar{u}_{n}\right)
$$

for every $\delta>0$. Hence, upon integrating along the interval $(0, t)$ we find

$$
\begin{aligned}
\frac{1}{2} \mathrm{~d}^{2}\left(w_{\varepsilon_{n}}(t), w_{\varepsilon_{n}}(0)\right) & \leq \delta\left(\phi\left(w_{\varepsilon_{n}}(0)\right)-\phi\left(w_{\varepsilon_{n}}(t)\right)\right)+\frac{1}{\delta} \int_{0}^{t} \frac{1}{2} \mathrm{~d}^{2}\left(w_{\varepsilon_{n}}(s), w_{\varepsilon_{n}}(0)\right) \mathrm{d} s \\
& \leq C+\mathrm{B} \delta \mathrm{d}^{2}\left(w_{\varepsilon_{n}}(t), \bar{u}_{n}\right)+\delta \mathrm{Q}\left(\bar{u}_{n}\right)+\frac{1}{\delta} \int_{0}^{t} \frac{1}{2} \mathrm{~d}^{2}\left(w_{\varepsilon_{n}}(s), \bar{u}_{n}\right) \mathrm{d} s
\end{aligned}
$$

where the first inequality follows from estimate (5.27), and the last one from the coercivity condition $(2.19 \mathrm{c})$ for $\phi$. Choosing $\delta=1 /(8 \mathrm{~B})$ and taking into account the bounds on the sequence $\left(\bar{u}_{n}\right)_{n}$, we then conclude

$$
\mathrm{d}^{2}\left(w_{\varepsilon_{n}}(t), \bar{u}_{n}\right) \leq C\left(1+\int_{0}^{t} \mathrm{~d}^{2}\left(w_{\varepsilon_{n}}(s), \bar{u}_{n}\right) \mathrm{d} s\right)
$$

whence, by the Gronwall Lemma,

$$
\sup _{n \in \mathbb{N}, t \in[0, \infty)} \mathrm{d}^{2}\left(w_{\varepsilon_{n}}(t), \bar{u}_{n}\right) \leq C .
$$

Combining this estimate with (2.20) we infer

$$
\exists C>0 \quad \forall n \in \mathbb{N} \forall t \in[0, \infty): \quad\left|\phi\left(w_{\varepsilon_{n}}(t)\right)\right| \leq C,
$$

whence by $(5.27)$

$$
\int_{0}^{t} \mathcal{H}_{\varepsilon_{n}}(s) \mathrm{d} s \leq C \quad \text { for all } t \in[0, \infty), n \in \mathbb{N}
$$

Therefore, we have

$$
\begin{aligned}
\frac{1}{\varepsilon_{n}} \int_{0}^{\infty}\left(\phi\left(\bar{u}_{n}\right)-\phi\left(w_{\varepsilon_{n}}(t)\right)\right) \mathrm{d} \mu_{\varepsilon_{n}}(t) & \geq \frac{1}{\varepsilon_{n}} \int_{0}^{\infty}\left(\int_{0}^{t} \mathcal{H}_{\varepsilon_{n}}(s) \mathrm{d} s\right) \mathrm{d} \mu_{\varepsilon_{n}}(t) \\
& =\frac{1}{\varepsilon_{n}} \int_{0}^{\infty} \mathrm{e}^{-t / \varepsilon_{n}} \mathcal{H}_{\varepsilon_{n}}(t) \mathrm{d} t
\end{aligned}
$$

where the second equality follows from the integration by parts formula (2.34), taking into account (5.28). Plugging (5.29) into (5.24), the term $\frac{1}{\varepsilon_{n}} \int_{0}^{\infty} \frac{\mathrm{e}^{-t / \varepsilon_{n}}}{2}\left|w_{\varepsilon_{n}}^{\prime}\right|^{2}(t) \mathrm{d} t$ cancels out, and we conclude that

$$
\frac{1}{\varepsilon_{n}}\left(\phi\left(\bar{u}_{n}\right)-V_{\varepsilon_{n}}\left(\bar{u}_{n}\right)\right) \geq \frac{1}{2} \int_{0}^{\infty}|\partial \phi|^{2}\left(w_{\varepsilon_{n}}(t)\right) \mathrm{d} \mu_{\varepsilon_{n}}(t)=\frac{1}{2} \int_{0}^{\infty} \mathrm{e}^{-s}|\partial \phi|^{2}\left(w_{\varepsilon_{n}}\left(\varepsilon_{n} s\right)\right) \mathrm{d} s,
$$

where again we have used the change of variables in (5.4). Since for all $s \in[0, \infty)$ we have

$$
\mathrm{d}\left(w_{\varepsilon_{n}}\left(\varepsilon_{n} s\right), w_{\varepsilon_{n}}(0)\right) \leq\left(\varepsilon_{n} s\right)^{1 / 2} \sup _{t \in[0, \infty)}\left\|w_{\varepsilon_{n}}^{\prime}\right\|_{L^{2}(0, t)} \leq C\left(\varepsilon_{n} s\right)^{1 / 2}
$$

(the latter estimate due to (5.27)) and $w_{\varepsilon_{n}}(0)=\bar{u}_{n} \stackrel{\sigma}{\rightarrow} \bar{u}$ as $n \rightarrow \infty$, we conclude that $w_{\varepsilon_{n}}\left(\varepsilon_{n} s\right) \stackrel{\sigma}{\rightarrow} \bar{u}$ as $n \rightarrow \infty$ for all $s \in[0, \infty)$. Also, observe that for all $s \in[0, \infty) \sup _{n \in \mathbb{N}} \mathrm{d}\left(w_{\varepsilon_{n}}\left(\varepsilon_{n} s\right), \bar{u}\right) \leq C$ due to the bounds on $\left(\bar{u}_{n}\right)_{n}$ and (5.31), and that $\sup _{\varepsilon} \phi\left(w_{\varepsilon_{n}}\left(\varepsilon_{n} s\right)\right) \leq C$ by (5.27). Therefore,

$$
\liminf _{n \rightarrow \infty}|\partial \phi|^{2}\left(w_{\varepsilon_{n}}\left(\varepsilon_{n} s\right)\right) \geq\left|\partial^{-} \phi\right|^{2}(\bar{u}) \quad \text { for all } s \in[0, \infty) .
$$


Ultimately, from (5.30) and Fatou's Lemma we find

$$
\begin{aligned}
\liminf _{n \rightarrow \infty} \frac{1}{\varepsilon_{n}}\left(\phi\left(\bar{u}_{n}\right)-V_{\varepsilon_{n}}\left(\bar{u}_{n}\right)\right) & \geq \frac{1}{2} \int_{0}^{\infty} \mathrm{e}^{-s} \liminf _{n \rightarrow \infty}|\partial \phi|^{2}\left(w_{\varepsilon_{n}}\left(\varepsilon_{n} s\right)\right) \mathrm{d} s \\
& \geq \frac{1}{2} \int_{0}^{\infty} \mathrm{e}^{-s}\left|\partial^{-} \phi\right|^{2}(\bar{u}) \mathrm{d} s=\frac{1}{2}\left|\partial^{-} \phi\right|^{2}(\bar{u}),
\end{aligned}
$$

whence $(5.25)$. Since the sequences $\left(\bar{u}_{n}\right)_{n}$ and $\left(\varepsilon_{n}\right)_{n}$ are arbitrary, we conclude $(5.22)$.

Finally, let us check that

$$
\left|\partial_{\mathrm{w}}^{-} \phi\right|(\bar{u}) \geq \mathscr{G}^{-}(\bar{u}) \quad \text { for all } \bar{u} \in \mathrm{D}(\phi),
$$

whence (5.23) immediately follows. With this aim, let us fix $\eta>0$ and pick a sequence $\left(\bar{u}_{n}\right)_{n}$ with $\bar{u}_{n} \stackrel{\sigma}{\rightarrow} \bar{u}$, and $\sup _{n}\left(\mathrm{~d}\left(\bar{u}_{n}, \bar{u}\right), \phi\left(\bar{u}_{n}\right)\right)<\infty$ such that

$$
\liminf _{n \rightarrow \infty} \limsup _{\varepsilon \downarrow 0} G_{\varepsilon}\left(\bar{u}_{n}\right) \leq\left|\partial_{\mathrm{w}}^{-} \phi\right|(\bar{u})+\eta .
$$

Up to an extraction, we may replace $\liminf _{n \rightarrow \infty}$ by $\lim _{n \rightarrow \infty}$. Hence,

$$
\exists \bar{n} \in \mathbb{N} \forall n \geq \bar{n}: \quad \limsup _{\varepsilon \downarrow 0} G_{\varepsilon}\left(\bar{u}_{n}\right)=\inf _{r>0} \sup _{\varepsilon \in(0, r)} G_{\varepsilon}\left(\bar{u}_{n}\right) \leq\left|\partial_{\mathrm{w}}^{-} \phi\right|(\bar{u})+2 \eta .
$$

Therefore, there exists a vanishing sequence $\left(r_{n}\right)_{n}$ such that, for $n$ sufficiently big, $G_{r_{n}}\left(\bar{u}_{n}\right) \leq$ $\left|\partial_{\mathrm{w}}^{-} \phi\right|(\bar{u})+2 \eta$. This ensures that

$$
\mathscr{G}^{-}(\bar{u}) \leq \liminf _{n \rightarrow \infty} G_{r_{n}}\left(\bar{u}_{n}\right) \leq\left|\partial_{\mathrm{w}}^{-} \phi\right|(\bar{u})+3 \eta,
$$

which concludes the proof of (5.32), since $\eta>0$ is arbitrary.

Combining Propositions 5.3 and 5.4 we conclude the following result, specifying in which sense the quantities $\left(G_{\varepsilon}\right)_{\varepsilon}$ approximate the relaxed slope $\left|\partial^{-} \phi\right|$.

Corollary 5.5. Under the LSCC Property 2.5 there holds

$$
\left|\partial_{\mathrm{w}}^{-} \phi\right|(\bar{u})=\left|\partial^{-} \phi\right|(\bar{u}) \quad \text { for every } \bar{u} \in \mathrm{D}(\phi) .
$$

In particular, if the local slope $|\partial \phi|$ is $\sigma$-lower semicontinuous along $\mathrm{d}$-bounded sequences with bounded energy, then $\left|\partial_{\mathrm{w}}^{-} \phi\right|(\bar{u})=|\partial \phi|(\bar{u})$ for all $\bar{u} \in \mathrm{D}(\phi)$.

5.3. Proof of Theorem 3.6. It follows from Corollary 4.4 and the fact that $\sup _{\varepsilon} \phi\left(\bar{u}_{\varepsilon}\right) \leq C$ that

$$
\exists C \geq 0 \quad \forall \varepsilon>0 \forall t \in[0, \infty): \quad\left\{\begin{array}{l}
\int_{0}^{t}\left|u_{\varepsilon}^{\prime}\right|^{2}(s) \mathrm{d} s \leq C, \\
\int_{0}^{t} \phi\left(u_{\varepsilon}(s)\right) \mathrm{d} s \leq C .
\end{array}\right.
$$

Moreover, observing that $\mathrm{d}\left(u_{\varepsilon}(t), \bar{u}_{\varepsilon}\right) \leq \int_{0}^{t}\left|u_{\varepsilon}^{\prime}\right|(s) \mathrm{d} s$ and taking into account (3.17), we conclude that for every $T>0$

$$
\exists C=C(T)>0 \quad \forall \varepsilon>0 \quad \forall t \in[0, T]: \quad \mathrm{d}\left(u_{\varepsilon}(t), \bar{u}\right) \leq C(T) .
$$

We now apply Theorem 2.6 and conclude that, for every vanishing $\left(\varepsilon_{k}\right)_{k}$ there exist a (not relabeled) subsequence $\left(u_{\varepsilon_{k}}\right)_{k}$ and $u \in \mathrm{AC}_{\mathrm{loc}}([0, \infty) ; X)$ such that the pointwise convergence (3.18) holds, as well as (2.23) and (2.24).

We are now in a position to pass to the limit as $\varepsilon_{k} \downarrow 0$ in identity (4.11), which we integrate on any interval $(0, t) \subset(0, \infty)$ :

$$
\frac{1}{2} \int_{0}^{t}\left|u_{\varepsilon_{k}}^{\prime}\right|^{2}(s) \mathrm{d} s+\int_{0}^{t} \frac{1}{\varepsilon_{k}}\left(\phi\left(u_{\varepsilon_{k}}(s)\right)-V_{\varepsilon_{k}}\left(u_{\varepsilon_{k}}(s)\right)\right) \mathrm{d} s+V_{\varepsilon_{k}}\left(u_{\varepsilon_{k}}(t)\right)=V_{\varepsilon_{k}}\left(\bar{u}_{\varepsilon_{k}}\right) .
$$

Assumption (3.17), estimate (4.3), and the lim inf-inequality (5.3) yield that

$$
\phi(\bar{u}) \leq \liminf _{k \rightarrow \infty} V_{\varepsilon_{k}}\left(\bar{u}_{\varepsilon_{k}}\right) \leq \limsup _{k \rightarrow \infty} V_{\varepsilon_{k}}\left(\bar{u}_{\varepsilon_{k}}\right) \leq \limsup _{k \rightarrow \infty} \phi\left(\bar{u}_{\varepsilon_{k}}\right)=\phi(\bar{u}) .
$$

As for the left-hand side of (5.35), we observe that

$$
\left.\liminf _{\varepsilon_{k} \downarrow 0} V_{\varepsilon_{k}}\left(u_{\varepsilon_{k}}(t)\right) \geq \phi(u(t))\right) \quad \text { for all } t \in[0, \infty)
$$


thanks to (5.3) and (3.18), and

$$
\liminf _{\varepsilon_{k} \downarrow 0} \int_{0}^{t}\left|u_{\varepsilon_{k}}^{\prime}\right|^{2}(s) \mathrm{d} s \geq \int_{0}^{t}\left|u^{\prime}\right|^{2}(s) \mathrm{d} s .
$$

by (2.23). In order to conclude (3.19), it remains to show that

$$
\liminf _{\varepsilon_{k} \downarrow 0} \int_{0}^{t} \frac{1}{\varepsilon_{k}}\left(\phi\left(u_{\varepsilon_{k}}(s)\right)-V_{\varepsilon_{k}}\left(u_{\varepsilon_{k}}(s)\right)\right) \mathrm{d} s \geq \int_{0}^{t} \frac{1}{2}\left|\partial^{-} \phi\right|^{2}(u(s)) \mathrm{d} s .
$$

With this aim, we use that, for any $\delta>0$

$$
\begin{aligned}
\liminf _{\varepsilon_{k} \downarrow 0} \int_{0}^{t} \frac{1}{\varepsilon_{k}}\left(\phi\left(u_{\varepsilon_{k}}(s)\right)-V_{\varepsilon_{k}}\left(u_{\varepsilon_{k}}(s)\right)\right) \mathrm{d} s \geq & \liminf _{\varepsilon_{k} \downarrow 0} \int_{0}^{t}\left(\frac{\phi\left(u_{\varepsilon_{k}}(s)\right)-V_{\varepsilon_{k}}\left(u_{\varepsilon_{k}}(s)\right)}{\varepsilon_{k}}+\delta \phi\left(u_{\varepsilon_{k}}(s)\right)\right) \mathrm{d} s \\
& +\liminf _{\varepsilon_{k} \downarrow 0}\left(-\delta \int_{0}^{t} \phi\left(u_{\varepsilon_{k}}(s)\right) \mathrm{d} s\right)=: I_{1}+I_{2} .
\end{aligned}
$$

Now,

$$
I_{2}=-\delta \limsup _{\varepsilon_{k} \downarrow 0} \int_{0}^{t} \phi\left(u_{\varepsilon_{k}}(s)\right) \mathrm{d} s \geq-\delta C
$$

for a constant independent of $\varepsilon_{k}$, where the latter inequality ensues from estimate (4.15b) and condition (3.17). As for $I_{1}$, we may apply the Fatou Lemma since the function

$$
s \mapsto \frac{\phi\left(u_{\varepsilon_{k}}(s)\right)-V_{\varepsilon_{k}}\left(u_{\varepsilon_{k}}(s)\right)}{\varepsilon_{k}}+\delta \phi\left(u_{\varepsilon_{k}}(s)\right)
$$

is bounded from below by a constant independent of $\varepsilon_{k}$ : indeed, the first summand is positive, and the second one is bounded from below in view of the coercivity (2.19c) and estimate (5.34) above. Therefore,

$$
I_{1} \geq \int_{0}^{t} \liminf _{\varepsilon_{k} \downarrow 0}\left(\frac{\phi\left(u_{\varepsilon_{k}}(s)\right)-V_{\varepsilon_{k}}\left(u_{\varepsilon_{k}}(s)\right)}{\varepsilon_{k}}+\delta \phi\left(u_{\varepsilon_{k}}(s)\right)\right) \mathrm{d} s
$$

Now, for any fixed $s \in(0, t)$ out of a negligible set, let us extract a further subsequence $\left(\varepsilon_{k}^{\prime}\right)$, possibly depending on $s$, such that

$$
\liminf _{\varepsilon_{k} \downarrow 0}\left(\frac{\phi\left(u_{\varepsilon_{k}}(s)\right)-V_{\varepsilon_{k}}\left(u_{\varepsilon_{k}}(s)\right)}{\varepsilon_{k}}+\delta \phi\left(u_{\varepsilon_{k}}(s)\right)\right)=\lim _{\varepsilon_{k}^{\prime} \downarrow 0}\left(\frac{\phi\left(u_{\varepsilon_{k}^{\prime}}(s)\right)-V_{\varepsilon_{k}^{\prime}}\left(u_{\varepsilon_{k}^{\prime}}(s)\right)}{\varepsilon_{k}^{\prime}}+\delta \phi\left(u_{\varepsilon_{k}^{\prime}}(s)\right)\right) .
$$

Observe that, along this subsequence there holds $\sup _{k} \phi\left(u_{\varepsilon_{k}^{\prime}}(s)\right)<\infty$, as well as estimate (5.34) and convergence (3.18). Therefore, we are in the position to apply the $\Gamma$-lim inf inequality $(5.22)$ from Lemma 5.4. We ultimately conclude that

$$
\liminf _{\varepsilon_{k} \downarrow 0}\left(\frac{\phi\left(u_{\varepsilon_{k}}(s)\right)-V_{\varepsilon_{k}}\left(u_{\varepsilon_{k}}(s)\right)}{\varepsilon_{k}}+\delta \phi\left(u_{\varepsilon_{k}}(s)\right)\right) \geq \frac{1}{2}\left|\partial^{-} \phi\right|^{2}(u(s))+\delta \phi(u(s)) \quad \text { for a.a. } s \in(0, t) .
$$

All in all, we deduce that

$$
\liminf _{\varepsilon_{k} \downarrow 0} \int_{0}^{t} \frac{1}{\varepsilon_{k}}\left(\phi\left(u_{\varepsilon_{k}}(s)\right)-V_{\varepsilon_{k}}\left(u_{\varepsilon_{k}}(s)\right)\right) \mathrm{d} s \geq \int_{0}^{t} \frac{1}{2}\left|\partial^{-} \phi\right|^{2}(u(s)) \mathrm{d} s+\delta \int_{0}^{t} \phi(u(s)) \mathrm{d} s-C \delta .
$$

Since $\delta$ is arbitrary, we infer (5.39).

Combining (5.36)-(5.39) we pass to the limit in (5.35) and thus conclude the proof of the integral inequality (3.19).

\section{Finer Results For $\lambda$-GeOdesically CONVEX EnERgies}

Throughout this section, we shall further assume that

$$
\phi \text { is } \lambda \text {-geodesically convex on } X \text { for some } \lambda \in \mathbb{R} \text {, }
$$

cf. (2.9). Under this condition, first of all we shall prove the continuity of the value function with respect to the metric $\mathrm{d}$. The following result complements Lemma 4.1, where we showed the sequential $\sigma$-lower semicontinuity of $V_{\varepsilon}$ on d-bounded sets, as well as Theorem A.6 in Appendix A ahead. 
Lemma 6.1. Assume Property 2.5 and (6.1). Then, $V_{\varepsilon}$ is continuous on sublevels of the energy $\phi$, namely

$$
\left(\bar{u}_{n} \rightarrow \bar{u} \text { and } \sup _{n} \phi\left(\bar{u}_{n}\right)<\infty\right) \Rightarrow V_{\varepsilon}\left(\bar{u}_{n}\right) \rightarrow V_{\varepsilon}(\bar{u}) .
$$

Proof. Let $u_{\varepsilon} \in \mathscr{C}_{\varepsilon}(\bar{u})$ be a minimizer for $\mathcal{I}_{\varepsilon}$ (observe that it exists since $\bar{u} \in \mathrm{D}(\phi)$ ). We construct a sequence of curves $\left(u_{n}\right)_{n}$ with $u_{n} \in \mathscr{C}_{\varepsilon}\left(\bar{u}_{n}\right)$ for every $n \in \mathbb{N}$, fulfilling

$$
\limsup _{n \rightarrow \infty} V_{\varepsilon}\left(\bar{u}_{n}\right) \leq \limsup _{n \rightarrow \infty} \mathcal{I}_{\varepsilon}\left[u_{n}\right] \leq \mathcal{I}_{\varepsilon}\left[u_{\varepsilon}\right]=V_{\varepsilon}(\bar{u})
$$

and combine this with the previously proved lower semicontinuity of $V_{\varepsilon}$ with respect to the topology $\sigma$, cf. Lemma 4.1. To construct $\left(u_{n}\right)_{n}$, we argue in this way: for every $n \in \mathbb{N}$ we set $\tau_{n}:=\mathrm{d}\left(\bar{u}_{n}, \bar{u}\right)$, and consider the constant-speed geodesic $\gamma_{n}:\left[0, \tau_{n}\right] \rightarrow X$ connecting $\bar{u}_{n}$ to $\bar{u}$, such that

$$
\frac{\mathrm{d}\left(\gamma_{n}(t), \gamma_{n}(s)\right)}{t-s}=1 \quad \text { for all } s, t \in\left[0, \tau_{n}\right] .
$$

Hence $\left|\gamma_{n}^{\prime}\right|(t)=1$ for almost all $t \in\left(0, \tau_{n}\right)$. We define $u_{n}:[0, \infty) \rightarrow X$ setting

$$
u_{n}(t):= \begin{cases}\gamma_{n}(t) & t \in\left[0, \tau_{n}\right], \\ u_{\varepsilon}(t) & t \in\left[\tau_{n}, \infty\right)\end{cases}
$$

Then,

$$
\mathcal{I}_{\varepsilon}\left[u_{n}\right]=\int_{0}^{\tau_{n}} \ell_{\varepsilon}\left(t, \gamma_{n}(t),\left|\gamma_{n}^{\prime}\right|(t)\right) \mathrm{d} t+\int_{\tau_{n}}^{\infty} \ell_{\varepsilon}\left(t, u_{\varepsilon}(t),\left|u_{\varepsilon}^{\prime}\right|(t)\right) \mathrm{d} t=: I_{1}+I_{2}
$$

Since $I_{2}$ converges to $\mathcal{I}_{\varepsilon}\left[u_{\varepsilon}\right]$ as $n \rightarrow \infty$, to conclude (6.3) it remains to show that $\lim _{n \rightarrow \infty} I_{1}=0$. Now, by (6.4) and the $\lambda$-convexity (6.1) we have

$$
\begin{aligned}
I_{1} & =\int_{0}^{\tau_{n}} \mathrm{e}^{-t / \varepsilon}\left(\frac{1}{2}+\frac{1}{\varepsilon} \phi\left(\gamma_{n}(t)\right)\right) \mathrm{d} t \\
& \leq \varepsilon\left(1-\mathrm{e}^{-\tau_{n} / \varepsilon}\right)+\max \left\{\phi\left(\bar{u}_{n}\right), \phi(\bar{u})\right\}\left(1-\mathrm{e}^{-\tau_{n} / \varepsilon}\right)-\frac{\lambda}{2} \mathrm{~d}^{2}\left(\bar{u}, \bar{u}_{n}\right) \int_{0}^{\tau_{n}} \mathrm{e}^{-t / \varepsilon} \frac{\left(\tau_{n}-t\right) t}{\tau_{n}^{2}} \mathrm{~d} t,
\end{aligned}
$$

and we refer to the last integral as $I_{3}$. We have $\lim _{n \rightarrow \infty} I_{3}=0$, hence the right-hand side in the above inequality converges to 0 as $n \rightarrow \infty$, which concludes the proof.

In the following two sections we are going to provide a series of finer properties, and estimates, for the family $\left(u_{\varepsilon}\right)_{\varepsilon}$ of WED-minimizers. We shall prove them under the $\lambda$-convexity condition (6.1), distinguishing the cases $\lambda=0$, handled in the upcoming Section 6.1 , and $\lambda<0$, see Sec. 6.2. The starting point for all calculations will be the following relation

$-\varepsilon \frac{\mathrm{d}^{2}}{\mathrm{~d} t^{2}}\left(\frac{1}{2}\left|u_{\varepsilon}^{\prime}\right|^{2}(t)\right)+\frac{\mathrm{d}}{\mathrm{d} t} \frac{1}{2}\left|u_{\varepsilon}^{\prime}\right|^{2}(t)=-\frac{\mathrm{d}^{2}}{\mathrm{~d} t^{2}} \phi\left(u_{\varepsilon}(t)\right)-\frac{\mathrm{d}}{\mathrm{d} t} \frac{1}{2}\left|u_{\varepsilon}^{\prime}\right|^{2}(t) \leq-\lambda\left|u_{\varepsilon}^{\prime}\right|^{2}(t)$ in $\mathcal{D}^{\prime}(0, \infty)$,

holding for all $\lambda \leq 0$.

Remark 6.2. In the Euclidean case $X=\mathbb{R}^{n}$, for $\phi$ smooth, we can formally derive (6.5) by testing by $u_{\varepsilon}^{\prime}$ the Euler-Lagrange equation satisfied by WED-minimizers, i.e. $-\varepsilon u_{\varepsilon}{ }^{\prime \prime}+u_{\varepsilon}^{\prime}+\mathrm{D} \phi\left(u_{\varepsilon}\right)=0$, and differentiating the relation thus obtained. Therefore,

$$
\begin{aligned}
-\frac{\varepsilon}{2} \frac{\mathrm{d}}{\mathrm{d} t^{2}}\left|u_{\varepsilon}^{\prime}(t)\right|^{2}+\frac{\mathrm{d}}{\mathrm{d} t}\left|u_{\varepsilon}^{\prime}(t)\right|^{2} & =-\frac{\mathrm{d}}{\mathrm{d} t}\left(\left\langle\mathrm{D} \phi\left(u_{\varepsilon}(t)\right), u_{\varepsilon}^{\prime}(t)\right\rangle\right) \\
& =-\left\langle\mathrm{D} \phi\left(u_{\varepsilon}(t)\right), u_{\varepsilon}^{\prime \prime}(t)\right\rangle-\left\langle\mathrm{D}^{2} \phi\left(u_{\varepsilon}(t)\right)\left(u_{\varepsilon}^{\prime}(t)\right), u_{\varepsilon}^{\prime}(t)\right\rangle \\
& \leq-\varepsilon\left|u_{\varepsilon}^{\prime \prime}(t)\right|^{2}+\frac{1}{2} \frac{\mathrm{d}}{\mathrm{d} t}\left|u_{\varepsilon}^{\prime}(t)\right|^{2}-\lambda\left|u_{\varepsilon}^{\prime}(t)\right|^{2} \\
& \leq \frac{1}{2} \frac{\mathrm{d}}{\mathrm{d} t}\left|u_{\varepsilon}^{\prime}(t)\right|^{2}-\lambda\left|u_{\varepsilon}^{\prime}(t)\right|^{2}
\end{aligned}
$$

where for the first inequality we have used that $-\mathrm{D} \phi\left(u_{\varepsilon}\right)=-\varepsilon u_{\varepsilon}^{\prime \prime}+u_{\varepsilon}^{\prime}$ by the Euler-Lagrange equation, and that $\phi \lambda$-convex implies $\mathrm{D}^{2} \phi \geq \lambda$. Therefore we conclude (6.5). 
In both Section 6.1 and Sec. 6.2, we will devote some effort to the proof of inequality (6.5) in the present metric context, where the above arguments are not available. Then, from (6.5) we shall deduce the additional properties of the WED-minimizers $\left(u_{\varepsilon}\right)_{\varepsilon}$.

The basic result underlying (6.5) is the following Lemma, which holds both for $\lambda=0$ and $\lambda<0$ and is thus anticipated here.

Lemma 6.3. Assume Property 2.5 and the $\lambda$-convexity (6.1) with $\lambda \in \mathbb{R}$. Set

$$
\mathcal{U}_{\varepsilon}(t):=\int_{0}^{t} \frac{1}{2}\left|u_{\varepsilon}^{\prime}\right|^{2}(s) \mathrm{d} s
$$

and for every $0 \leq a<b<\infty$ consider the family of linear functions

$$
\mathrm{I}_{a, b}(t):=\frac{t-a}{b-a}
$$

Then, for every $[a, b] \subset[0, \infty)$ we have

$$
\begin{aligned}
\int_{a}^{b}\left(\phi\left(u_{\varepsilon}(t)\right)+\mathcal{U}_{\varepsilon}(t)\right) \mathrm{d} \mu_{\varepsilon}(t) \leq & \left(\phi\left(u_{\varepsilon}(a)\right)+\mathcal{U}_{\varepsilon}(a)\right)\left(i_{a, b}-\theta_{a, b}\right)+\left(\phi\left(u_{\varepsilon}(b)\right)+\mathcal{U}_{\varepsilon}(b)\right) \theta_{a, b} \\
& -\frac{\lambda}{2} \mathrm{~d}^{2}\left(u_{\varepsilon}(a), u_{\varepsilon}(b)\right) \Gamma_{a, b}
\end{aligned}
$$

where $\theta_{a, b}:=\int_{a}^{b} \mathrm{I}_{a, b}(t) \mathrm{d} \mu_{\varepsilon}(t), i_{a, b}:=\mu_{\varepsilon}([a, b])$ and $\Gamma_{a, b}:=\int_{a}^{b} \mathrm{I}_{a, b}(t)\left(1-\mathrm{I}_{a, b}(t)\right) \mathrm{d} \mu_{\varepsilon}(t)$.

Proof. Let us take $a<b$ in $(0, \infty)$ such that $\phi\left(u_{\varepsilon}(a)\right)<\infty$ and $\phi\left(u_{\varepsilon}(b)\right)<\infty$ and consider the geodesic $\gamma:[a, b] \longrightarrow X$ connecting $u_{\varepsilon}(a)$ and $u_{\varepsilon}(b)$ with constant speed $\left|\gamma^{\prime}\right|(t)=\frac{\mathrm{d}\left(u_{\varepsilon}(a), u_{\varepsilon}(b)\right)}{b-a}$. Let us consider the curve $\tilde{v}$ defined by

$$
\tilde{v}(t)= \begin{cases}u_{\varepsilon}(t) & t \in(0, a) \text { or } t \in(b, \infty) \\ \gamma(t) & t \in[a, b]\end{cases}
$$

By construction, $\tilde{v} \in \mathscr{C}_{\varepsilon}(\bar{u})$, hence $\mathcal{I}_{\varepsilon}\left[u_{\varepsilon}\right] \leq \mathcal{I}_{\varepsilon}[\tilde{v}]$, which implies

$$
\int_{a}^{b}\left(\frac{\varepsilon}{2}\left|u_{\varepsilon}^{\prime}\right|^{2}(t)+\phi\left(u_{\varepsilon}(t)\right)\right) \mathrm{d} \mu_{\varepsilon}(t) \leq \int_{a}^{b}\left(\frac{\varepsilon}{2}\left|\gamma^{\prime}\right|^{2}(t)+\phi(\gamma(t))\right) \mathrm{d} \mu_{\varepsilon}(t) .
$$

Now, since $\phi$ is geodesically convex, there holds that

$\phi(\gamma(t)) \leq\left(1-\mathrm{I}_{a, b}(t)\right) \phi\left(u_{\varepsilon}(a)\right)+\mathrm{I}_{a, b}(t) \phi\left(u_{\varepsilon}(b)\right)-\frac{\lambda}{2}\left(1-\mathrm{I}_{a, b}(t)\right) \mathrm{I}_{a, b}(t) \mathrm{d}^{2}\left(u_{\varepsilon}(a), u_{\varepsilon}(b)\right)$ for all $t \in[a, b]$.

Moreover, we can estimate the speed of the geodesic $\gamma$ by

$$
\left|\gamma^{\prime}\right|^{2}(t)=\frac{\mathrm{d}^{2}\left(u_{\varepsilon}(a), u_{\varepsilon}(b)\right)}{(b-a)^{2}} \leq \frac{1}{b-a} \int_{a}^{b}\left|u_{\varepsilon}^{\prime}\right|^{2}(t) \mathrm{d} t \leq 2 \frac{\mathcal{U}_{\varepsilon}(b)-\mathcal{U}(a)}{b-a} .
$$

Now, we introduce the function

$$
\tilde{\mathcal{U}}_{\varepsilon}^{a, b}(t)= \begin{cases}\mathcal{U}_{\varepsilon}(t) & t \in(0, a) \text { or } t \in(b, \infty), \\ \left(1-\mathrm{I}_{a, b}(t)\right) \mathcal{U}_{\varepsilon}(a)+\mathrm{I}_{a, b}(t) \mathcal{U}_{\varepsilon}(b) & t \in[a, b]\end{cases}
$$

which coincides with $\mathcal{U}_{\varepsilon}$ when $t=a, b$ and satisfies $\frac{1}{2}\left|\gamma^{\prime}\right|^{2}(t) \leq \frac{\mathrm{d}}{\mathrm{d} t} \tilde{\mathcal{U}}_{\varepsilon}^{a, b}(t)$ for all $t \in(a, b)$. We have

$$
\begin{aligned}
& \varepsilon \int_{a}^{b} \frac{1}{2}\left|u_{\varepsilon}^{\prime}\right|^{2}(t) \mathrm{d} \mu_{\varepsilon}(t)=\int_{a}^{b} \mathcal{U}_{\varepsilon}(t) \mathrm{d} \mu_{\varepsilon}(t)+\left[\mathrm{e}^{-t / \varepsilon} \mathcal{U}_{\varepsilon}(t)\right]_{a}^{b}, \\
& \varepsilon \int_{a}^{b} \frac{1}{2}\left|\gamma^{\prime}\right|^{2}(t) \mathrm{d} \mu_{\varepsilon}(t) \leq \varepsilon \int_{a}^{b} \frac{\mathrm{d}}{\mathrm{d} t} \tilde{\mathcal{U}}_{\varepsilon}^{a, b}(t) \mathrm{d} \mu_{\varepsilon}(t)=\int_{a}^{b} \tilde{\mathcal{U}}_{\varepsilon}^{a, b}(t) \mathrm{d} \mu_{\varepsilon}(t)+\left[\mathrm{e}^{-t / \varepsilon} \tilde{\mathcal{U}}_{\varepsilon}^{a, b}\right]_{a}^{b},
\end{aligned}
$$

by the integration by parts formula (2.33), where the inequality in (6.13) is due to (6.11). Thus, recalling that $\tilde{\mathcal{U}}_{\varepsilon}^{a, b}(t)=\mathcal{U}_{\varepsilon}(t)$ for $t=a, b$, and combining (6.12)-(6.13) with (6.9) and (6.10), we deduce (6.8). 
We conclude this section by fixing an identity that can be checked with direct calculations, and that will have a crucial role in the following proofs:

$$
t^{2}=a^{2}\left(1-\mathrm{I}_{a, b}(t)\right)+b^{2} \mathrm{I}_{a, b}(t)-(b-a)^{2}\left(1-\mathrm{I}_{a, b}(t)\right) \mathbf{I}_{a, b}(t) \text { for all } t \in[a, b] \text { and all } 0 \leq a<b .
$$

6.1. Finer properties of WED minimizers in the $\lambda$-convex case, $\lambda=0$. The main result of this section, Theorem 6.4 below, shows that, for every fixed $\varepsilon>0$, along WED minimizers $u_{\varepsilon}$ the energy $\phi$ is nonincreasing and convex. Moreover, we also prove that the map $t \mapsto \phi\left(u_{\varepsilon}(t)\right)$ is continuous on $[0, \infty)$, i.e. it enjoys the same continuity as $u_{\varepsilon}$. Observe that these are the properties of $\phi$ along a curve of maximal slope, i.e. a solution of the gradient flow in the limit as $\varepsilon \downarrow 0$, cf. [Bré73, Thm. 3.2, page 57] for the Hilbertian case, and [AGS08, Thm. 2.4.15] in the metric context. Interestingly, and somewhat surprisingly, these properties hold also at the level $\varepsilon>0$, provided that the energy is geodesically convex.

Theorem 6.4. Assume Property 2.5, (6.1) with $\lambda=0$, and let $u_{\varepsilon} \in \mathcal{M}_{\varepsilon}(\bar{u})$. Then,

(1) $t \mapsto \frac{1}{2}\left|u_{\varepsilon}^{\prime}\right|^{2}(t)$ admits a locally Lipschitz continuous pointwise representative on $(0, \infty)$;

(2) $t \mapsto\left|u_{\varepsilon}^{\prime}\right|(t)$ and $t \mapsto \phi\left(u_{\varepsilon}(t)\right)$ are nonincreasing,

(3) $t \mapsto \phi\left(u_{\varepsilon}(t)\right)$ is convex,

(4) (6.5) holds with $\lambda=0$, i.e.

$$
-\varepsilon \frac{\mathrm{d}^{2}}{\mathrm{~d} t^{2}}\left(\frac{1}{2}\left|u_{\varepsilon}^{\prime}\right|^{2}(t)\right)+\frac{\mathrm{d}}{\mathrm{d} t} \frac{1}{2}\left|u_{\varepsilon}^{\prime}\right|^{2}(t)=-\frac{\mathrm{d}^{2}}{\mathrm{~d} t^{2}} \phi\left(u_{\varepsilon}(t)\right)-\frac{\mathrm{d}}{\mathrm{d} t} \frac{1}{2}\left|u_{\varepsilon}^{\prime}\right|^{2}(t) \leq 0 \quad \text { in } \mathcal{D}^{\prime}(0, \infty) .
$$

Hence, the function $t \mapsto \phi\left(u_{\varepsilon}(t)\right)$ is continuous on $(0, \infty)$ and right-continuous at $t=0$.

For the proof of Theorem 6.4 we need a series of auxiliary results. The first one will allow us to deduce from estimate (6.8) (with $\lambda=0$ ) in Lemma 6.3 that the function $t \mapsto \phi\left(u_{\varepsilon}(t)\right)+\mathcal{U}_{\varepsilon}(t)$, with $\mathcal{U}_{\varepsilon}$ from (6.6), is convex.

Lemma 6.5. Let $\zeta \in C^{1}([0, \infty))$ be strictly positive and let $\psi$ be lower semicontinuous in $(0, \infty)$. If

$$
\int_{a}^{b} \psi(t) \zeta(t) \mathrm{d} t \leq \psi(a)\left(i_{a, b}-\theta_{a, b}\right)+\psi(b) \theta_{a, b} \quad \text { with }\left\{\begin{array}{l}
\theta_{a, b}:=\int_{a}^{b} \zeta(t) \mathbf{l}_{a, b}(t) \mathrm{d} t \\
i_{a, b}:=\int_{a}^{b} \zeta(t) \mathrm{d} t
\end{array}\right.
$$

then $\psi$ is convex.

Proof. We preliminarily prove that for any $\bar{t} \in(0, \infty)$

$$
\psi(\bar{t})=\liminf _{t \rightarrow \bar{t}} \psi(t) .
$$

Indeed, for any fixed $\bar{t}$ the lower semicontinuity of $\psi$ gives $\psi(\bar{t}) \leq \liminf _{t \downarrow \bar{t}} \psi(t)=: L$. Then, consider a sequence $t_{n} \downarrow \bar{t}$ for which $\psi\left(t_{n}\right) \rightarrow L$ as $n \rightarrow \infty$. Now, denoting by $\eta$ the measure $\eta:=\zeta(t) \mathcal{L}^{1}$, we have

$$
\begin{aligned}
& \liminf _{n \rightarrow \infty} \frac{1}{\eta\left(\left[\bar{t}, t_{n}\right]\right)} \int_{\bar{t}}^{t_{n}} \psi(t) \mathrm{d} \eta(t) \geq L \quad \text { and } \\
& \lim _{n \rightarrow \infty} \frac{1}{\eta\left(\left[\bar{t}, t_{n}\right]\right)} \int_{\bar{t}}^{t_{n}} \mathrm{I}_{\bar{t}, t_{n}}(t) \mathrm{d} \eta(t)=\lim _{n \rightarrow \infty} \frac{1}{\eta\left(\left[\bar{t}, t_{n}\right]\right)} \int_{\bar{t}}^{t_{n}}\left(1-\mathrm{I}_{\bar{t}, t_{n}}(t)\right) \mathrm{d} \eta(t)=\frac{1}{2}
\end{aligned}
$$

(recall the notation $\mathrm{I}_{\bar{t}, t_{n}}(t)=\frac{t-\bar{t}}{t_{n}-t}$ ). Thus, dividing both sides of (6.16) (written on the interval $\left.\left(\bar{t}, t_{n}\right)\right)$, by $\mu\left(\left[\bar{t}, t_{n}\right]\right)$ and letting $n \rightarrow \infty$, we get

$$
L \leq \frac{1}{2} \psi(\bar{t})+\frac{1}{2} L
$$

which, together with $\psi(\bar{t}) \leq L$, implies $L=\psi(\bar{t})$. The same argument works with a sequence $t_{n} \uparrow \bar{t}$, and we conclude (6.17).

Now, in order to conclude the proof we argue by contradiction. Thus, assume that $\psi$ is not convex. Then, there exist $\alpha<\bar{t}<\beta$ such that

$$
\psi(\bar{t})>\left(1-\mathrm{I}_{\alpha, \beta}(\bar{t})\right) \psi(\alpha)+\mathrm{I}_{\alpha, \beta}(\bar{t}) \psi(\beta) .
$$


Denote by $A_{\alpha, \beta}$ the open set defined as $A_{\alpha, \beta}:=\{t:(6.18)$ holds for $\alpha, \beta\}$. Let $(a, b)$ be the connected component of $A_{\alpha, \beta}$ containing $\bar{t}$. Thanks to (6.17) and to the lower semicontinuity of $\psi$, we have

$$
\psi(a)=\liminf _{t \rightarrow a} \psi(t) \geq\left(1-\mathrm{I}_{\alpha, \beta}(a)\right) \psi(\alpha)+\mathrm{I}_{\alpha, \beta}(a) \psi(\beta) \geq \psi(a),
$$

which gives $\psi(a)=\left(1-\mathrm{I}_{\alpha, \beta}(a)\right) \psi(\alpha)+\mathrm{I}_{\alpha, \beta}(a) \psi(\beta)$. The same argument also gives $\psi(b)=$ $\left(1-\mathrm{I}_{\alpha, \beta}(b)\right) \psi(\alpha)+\mathrm{I}_{\alpha, \beta}(b) \psi(\beta)$. Therefore, we can conclude that

$$
\psi(t)>\left(1-\mathrm{I}_{a, b}(t)\right) \psi(a)+\mathrm{I}_{a, b}(t) \psi(b) \text { for all } t \in(a, b) .
$$

Now, integrating the above inequality with respect to the measure $\mu$, we contradict (6.16).

We now derive a bound on the energy $\phi$ evaluated along $u_{\varepsilon}$ in terms of the initial energy.

Lemma 6.6. Let $u_{\varepsilon} \in \mathcal{M}_{\varepsilon}(\bar{u})$, then

$$
\phi\left(u_{\varepsilon}(t)\right) \leq \phi(\bar{u}) \quad \text { for all } t \geq 0 .
$$

Proof. By contradiction, assume that there exists a point $\bar{t}$ for which $\phi\left(u_{\varepsilon}(\bar{t})\right)>\phi(\bar{u})$. Since $\phi$ is lower semicontinuous, the set $A:=\left\{t \in(0, \infty): \phi\left(u_{\varepsilon}(t)\right)>\phi(\bar{u})\right\}$ is open. Let $(a, b)$ denote the connected component of $A$ containing $\bar{t}$ : then, $(a, b)$ is a bounded (open) interval of $(0, \infty)$. First of all $a \geq 0$. Moreover, $b$ is finite. In fact, assuming the opposite, we would have $\phi\left(u_{\varepsilon}(t)\right)>$ $\phi(\bar{u}) \geq \phi(a)$ for all $t \in(a, \infty)$, and thus, setting

$$
\tilde{u}(t)= \begin{cases}u_{\varepsilon}(t) \quad t \in(0, a) \text { or } t \in(b, \infty), \\ u_{\varepsilon}(a) \quad t \in[a, \infty),\end{cases}
$$

we would have $\mathcal{I}_{\varepsilon}\left[u_{\varepsilon}\right]>\mathcal{I}_{\varepsilon}[\tilde{u}]$, against the fact that $u_{\varepsilon}$ is a minimizer for $\mathcal{I}_{\varepsilon}$. Thus, $b<\infty$ and $\phi\left(u_{\varepsilon}(b)\right) \leq \phi(\bar{u})$.

Now, let us consider a geodesic $\tilde{\gamma}:[0,1] \longrightarrow X$ connecting $u_{\varepsilon}(a)$ with $u_{\varepsilon}(b)$ with unit speed. The convexity of $\phi$ implies that

$$
\phi(\tilde{\gamma}(s)) \leq \max \left\{\phi\left(u_{\varepsilon}(a)\right), \phi\left(u_{\varepsilon}(b)\right)\right\} \leq \phi(\bar{u}),
$$

where we have also used that $\phi\left(u_{\varepsilon}(b)\right) \leq \phi(\bar{u})$. We reparametrize the geodesic $\tilde{\gamma}$ on $[a, b]$ to a curve $\gamma$, with $\gamma(t):=\tilde{\gamma}(s(t))$, fulfilling $\left|\gamma^{\prime}\right|(t)=\left|u_{\varepsilon}^{\prime}\right|(t)$ on $[a, b]$. To obtain this, we consider the parametrization $t \mapsto s(t)$ such that

$$
s^{\prime}(t)=\left|u_{\varepsilon}^{\prime}\right|(t) \quad \text { and } \quad s(t):=\min \left\{\int_{a}^{t}\left|u_{\varepsilon}^{\prime}\right|(r) d r, 1\right\} .
$$

As a consequence, the curve

$$
v(t):= \begin{cases}u_{\varepsilon}(t) & t \in(0, a) \text { or } t \in(b, \infty) \\ \gamma(t) & t \in(a, b)\end{cases}
$$

satisfies

$$
\begin{aligned}
\int_{a}^{b}\left(\frac{\varepsilon}{2}\left|v^{\prime}\right|^{2}(t)+\phi(v(t))\right) \mathrm{d} \mu_{\varepsilon}(t) & =\int_{a}^{b}\left(\frac{\varepsilon}{2}\left|\gamma^{\prime}\right|^{2}(t)+\phi(v(t))\right) \mathrm{d} \mu_{\varepsilon}(t) \\
& <\int_{a}^{b}\left(\frac{\varepsilon}{2}\left|u_{\varepsilon}^{\prime}\right|^{2}(t)+\phi\left(u_{\varepsilon}(t)\right)\right) \mathrm{d} \mu_{\varepsilon}(t),
\end{aligned}
$$

which contradicts the minimality of $u_{\varepsilon}$ for $\mathcal{I}_{\varepsilon}$. Hence, (6.19) holds.

We now have all the ingredients for checking Theorem 6.4. 
Proof of Theorem 6.4. We split the proof in some steps.

$\triangleright(6.15)$ : First of all, Lemma 6.5 and inequality (6.8) give that $t \mapsto \phi\left(u_{\varepsilon}(t)\right)+\mathcal{U}_{\varepsilon}(t)$ is convex and thus

$$
\frac{\mathrm{d}^{2}}{\mathrm{~d} t^{2}}\left(\phi\left(u_{\varepsilon}(t)\right)+\mathcal{U}_{\varepsilon}(t)\right)=\frac{\mathrm{d}^{2}}{\mathrm{~d} t^{2}} \phi\left(u_{\varepsilon}(t)\right)+\frac{\mathrm{d}}{\mathrm{d} t} \frac{1}{2}\left|u_{\varepsilon}^{\prime}\right|^{2}(t) \geq 0 \quad \text { in } \mathcal{D}^{\prime}(0, \infty) .
$$

Then, we rewrite the metric inner variation equation (3.20) as

$$
\frac{\mathrm{d}}{\mathrm{d} t} \phi\left(u_{\varepsilon}(t)\right)+\frac{1}{2}\left|u_{\varepsilon}^{\prime}\right|^{2}(t)=\varepsilon \frac{\mathrm{d}}{\mathrm{d} t} \frac{1}{2}\left|u_{\varepsilon}^{\prime}\right|^{2}(t)-\frac{1}{2}\left|u_{\varepsilon}^{\prime}\right|^{2}(t) \quad \text { in } \mathcal{D}^{\prime}(0, \infty)
$$

which, together with (6.20), gives (6.15).

$\triangleright t \mapsto\left|u_{\varepsilon}^{\prime}\right|^{2}(t)$ is nonincreasing: To this end, we set $w_{\varepsilon}(t):=\frac{1}{2}\left|u_{\varepsilon}^{\prime}\right|^{2}(t)$. The above discussion immediately gives that $w_{\varepsilon}$ verifies

$$
-\varepsilon w_{\varepsilon}^{\prime \prime}+w_{\varepsilon}^{\prime} \leq 0 \quad \text { in } \mathcal{D}^{\prime}(0, \infty)
$$

which we rewrite as

$$
-\varepsilon \mathrm{e}^{t / \varepsilon} \frac{\mathrm{d}}{\mathrm{d} t}\left(\mathrm{e}^{-t / \varepsilon} w_{\varepsilon}^{\prime}\right) \leq 0 \quad \text { in } \mathcal{D}^{\prime}(0, \infty) .
$$

In fact, it follows from (6.23) that the distributional derivative $w_{\varepsilon}^{\prime}$ of $w_{\varepsilon}$ is locally bounded, so that $w_{\varepsilon}$ admits a locally Lipschitz pointwise representative, which will be still denoted by the same symbol. Moreover, the second distributional derivative $w_{\varepsilon}^{\prime \prime}$ is also locally bounded from above, so that $w_{\varepsilon}$ is semiconcave, and thus admits left and right derivatives at every point. We will use the right derivative $\left(w_{\varepsilon}\right)_{+}^{\prime}$ in the following argument to show that $w_{\varepsilon}$ is nonincreasing.

Indeed, suppose by contradiction that for some $\bar{t}$ we had $\left(w_{\varepsilon}\right)_{+}^{\prime}(\bar{t})>\bar{c}>0$. Since $t \mapsto \mathrm{e}^{-t / \varepsilon}\left(w_{\varepsilon}\right)_{+}^{\prime}$ is a nondecreasing function by $(6.23)$, for all $t \geq \bar{t}$ we would have $\mathrm{e}^{-t / \varepsilon}\left(w_{\varepsilon}\right)_{+}^{\prime}(t) \geq \mathrm{e}^{-\bar{t} / \varepsilon}\left(w_{\varepsilon}\right)_{+}^{\prime}(\bar{t}) \geq$ $\mathrm{e}^{-\bar{t} / \varepsilon} \bar{c}$, which would imply $w_{\varepsilon}(t) \geq w_{\varepsilon}(\bar{t})+\varepsilon \bar{c}\left(\mathrm{e}^{(t-\bar{t}) / \varepsilon}-1\right)$. This clearly contradicts the integrability of $w_{\varepsilon}(t)=\frac{1}{2}\left|u_{\varepsilon}^{\prime}\right|^{2}(t)$ on $(0, \infty)$ (cf. (4.15a)). Thus, we have obtained that $t \mapsto\left|u_{\varepsilon}^{\prime}\right|^{2}(t)$ is nonincreasing.

$\triangleright t \mapsto \phi\left(u_{\varepsilon}(t)\right)$ is convex: It follows from (6.15) and from the monotonicity of $t \mapsto\left|u_{\varepsilon}^{\prime}\right|^{2}(t)$ that $-\frac{\mathrm{d}^{2}}{\mathrm{~d} t^{2}} \phi\left(u_{\varepsilon}(t)\right) \leq 0$ in $\mathcal{D}^{\prime}(0, \infty)$, which yields the thesis.

$\triangleright$ continuity of $t \mapsto \phi\left(u_{\varepsilon}(t)\right)$ : it follows from the previously proved convexity that $t \mapsto \phi\left(u_{\varepsilon}(t)\right)$ is continuous on $(0, \infty)$. In order to check that $\phi \circ u_{\varepsilon}$ is right-continuous at $t=0$, we observe that, by $(6.19)$,

$$
\limsup _{t \downarrow 0} \phi\left(u_{\varepsilon}(t)\right) \leq \phi\left(u_{\varepsilon}(0)\right)=\phi(\bar{u}) .
$$

Since by $\left(2.19\right.$ a) we also have $\liminf _{t \downarrow 0} \phi\left(u_{\varepsilon}(t)\right) \geq \phi\left(u_{\varepsilon}(0)\right)$, we conclude that $\lim _{t \downarrow 0} \phi\left(u_{\varepsilon}(t)\right)=$ $\phi(\bar{u})$.

$\triangleright t \mapsto \phi\left(u_{\varepsilon}(t)\right)$ is nonincreasing: This follows from Lemma 6.6 and from the convexity of $t \mapsto$ $\phi\left(u_{\varepsilon}(t)\right)$.

6.2. Finer properties of WED minimizers in the $\lambda$-convex case, $\lambda<0$. The main result of this section is the analogue of Theorem 6.4 for $\lambda<0$. Observe that, along Hilbert and metric gradient flows (cf. the aforementioned [Bré73, Thm. 3.2, page 57], [AGS08, Thm. 2.4.15]), the map $\phi \circ u$ is nonincreasing and, if the energy $\phi$ is $\lambda$ (-geodesically) convex, $t \mapsto \mathrm{e}^{-2 \lambda^{-} t} \phi(u(t))$ is convex ( $\lambda^{-}$denoting the negative part of $\lambda$ ), and $t \mapsto \mathrm{e}^{2 \lambda t}\left|u^{\prime}\right|^{2}(t)$ is nonincreasing. Likewise, in Theorem 6.7 below we show that, at the level $\varepsilon>0$, along any WED minimizer $u_{\varepsilon}$ the functions $\phi \circ u_{\varepsilon}$ and $\left|u_{\varepsilon}^{\prime}\right|$ have these properties, with suitable correction terms.

Theorem 6.7. Assume the LSCC Property 2.5, (6.1) with $\lambda<0$, and let $u_{\varepsilon} \in \mathcal{M}_{\varepsilon}(\bar{u})$. Then,

(1) the function $t \mapsto \phi\left(u_{\varepsilon}(t)\right)$ is locally Lipschitz on $(0, \infty)$ and right-continuous at $t=0$;

(2) $t \mapsto \frac{1}{2}\left|u_{\varepsilon}^{\prime}\right|^{2}(t)$ admits a locally Lipschitz representative on $(0, \infty)$,

(3) (6.5) holds;

(4) $t \mapsto \phi\left(u_{\varepsilon}(t)\right)$ is nonincreasing;

(5) for every $[a, b] \subset(0, \infty)$ there exists $C_{a, b}>0$ such that $t \mapsto \phi\left(u_{\varepsilon}(t)\right)+\mathcal{U}_{\varepsilon}(t)$ (with $\mathcal{U}_{\varepsilon}$ from (6.6)) is $\lambda C_{a, b}$-convex on $[a, b]$. 
Moreover, for every $\lambda^{\prime}<\lambda$ there exists $\varepsilon^{\prime}>0$ such that for all $0<\varepsilon<\varepsilon^{\prime}$

(6) the function $t \mapsto \mathrm{e}^{2 \lambda^{\prime} t}\left|u_{\varepsilon}^{\prime}\right|^{2}(t)$ is nonincreasing.

We split the proof in several results, and start by checking the continuity of $t \mapsto \phi\left(u_{\varepsilon}(t)\right)$ on $(0, \infty)$, in Corollary 6.9 ahead, as a consequence of the result below, which establishes a suitable convexity-type property of the mapping $\phi \circ u_{\varepsilon}$.

Lemma 6.8. Assume the LSCC Property 2.5, (6.1) with $\lambda<0$, and let $u_{\varepsilon} \in \mathcal{M}_{\varepsilon}(\bar{u})$. Let us introduce the function

$$
L(t):=\int_{0}^{t}\left|u_{\varepsilon}^{\prime}\right|(r) \mathrm{d} r
$$

Then, there holds

$$
\phi\left(u_{\varepsilon}(t)\right) \leq(1-\mathrm{L}(a, b ; t)) \phi\left(u_{\varepsilon}(a)\right)+\mathrm{L}(a, b ; t) \phi\left(u_{\varepsilon}(b)\right)-\frac{\lambda}{2}(1-\mathrm{L}(a, b ; t)) \mathrm{L}(a, b ; t)(L(b)-L(a))^{2}
$$

for all $t \in[a, b]$ and all $[a . b] \subset(0, \infty)$, where we have used the short-hand notation, cf. (6.7),

$$
\mathrm{L}(a, b ; t):=\mathrm{I}_{L(a), L(b)}(L(t))=\frac{L(t)-L(a)}{L(b)-L(a)} .
$$

Therefore, the function $t \mapsto \phi\left(u_{\varepsilon}(t)\right)-\frac{\lambda}{2} L^{2}(t)$ is convex on $(0, \infty)$.

Proof. Preliminarily, we introduce the polynomial function

$$
P(s)=\phi\left(u_{\varepsilon}(a)\right)+(s-L(a)) \frac{\phi\left(u_{\varepsilon}(b)\right)-\phi\left(u_{\varepsilon}(a)\right)}{L(b)-L(a)}+\frac{\lambda}{2}(s-L(a))(L(b)-s),
$$

which satisfies $P(L(a))=\phi\left(u_{\varepsilon}(a)\right)$ and $P(L(b))=\phi\left(u_{\varepsilon}(b)\right)$ and $P^{\prime \prime}(s) \equiv-\lambda$. A direct calculation shows that

$$
\begin{aligned}
P(L(t))=(1 & -\mathrm{L}(\alpha, \beta ; t)) P(L(\alpha))+\mathrm{L}(\alpha, \beta ; t) P(L(\beta)) \\
& -\frac{\lambda}{2}(1-\mathrm{L}(\alpha, \beta ; t)) \mathrm{L}(\alpha, \beta ; t)(L(\beta)-L(\alpha))^{2} \quad \text { for all }[\alpha, \beta] \subset(0, \infty) .
\end{aligned}
$$

In particular,

$$
P(L(t))=(1-\mathrm{L}(a, b ; t)) \phi\left(u_{\varepsilon}(a)\right)+\mathrm{L}(a, b ; t) \phi\left(u_{\varepsilon}(b)\right)-\frac{\lambda}{2}(1-\mathrm{L}(a, b ; t)) \mathrm{L}(a, b ; t)(L(b)-L(a))^{2},
$$

so that $(6.25)$ reads

$$
\zeta(t):=\phi\left(u_{\varepsilon}(t)\right)-P(L(t)) \leq 0 \quad \text { for all } t \in[a, b] \text { for all }[a, b] \subset(0, \infty)
$$

Let us prove $(6.27)$ by contradiction. Suppose that there exist $[a, b] \subset(0, \infty)$ and $\bar{t} \in(a, b)$ (we may suppose that $\bar{t}$ is in the interior of $[a, b]$ by a lower semicontinuity argument), such that $\zeta(\bar{t})>0$. Denote by $A_{a, b}$ the subset of $[a, b]$ where $\zeta$ is strictly positive, and by $[\alpha, \beta]$ the connected component of $A_{a, b}$ containing $\bar{t}$, so that

$$
\zeta(t)>0 \quad \text { for all } t \in(\alpha, \beta), \zeta(\alpha)=\zeta(\beta)=0 .
$$

Arguing as in the proof of Lemma 6.3, we now choose a suitable competitor for the curve $u_{\varepsilon} \in$ $\mathcal{M}_{\varepsilon}(\bar{u})$ : we consider the geodesic $\gamma:[L(\alpha), L(\beta)] \rightarrow X$ connecting $u_{\varepsilon}(\alpha)$ to $u_{\varepsilon}(\beta)$ with unit speed, and define the curve

so that

$$
\tilde{v}(t):= \begin{cases}u_{\varepsilon}(t) & \text { if } t \in(0, \alpha) \text { or } t \in(\beta, \infty), \\ \gamma(L(t)) & \text { if } t \in[\alpha, \beta]\end{cases}
$$

$$
\left|\tilde{v}^{\prime}\right|(t)=\left|\gamma^{\prime}\right|(L(t)) L^{\prime}(t)=\left|u_{\varepsilon}^{\prime}\right|(t) \quad \text { for a.a. } t \in(\alpha, \beta) .
$$

From $\mathcal{I}_{\varepsilon}\left[u_{\varepsilon}\right] \leq \mathcal{I}_{\varepsilon}[\tilde{v}]$ we then conclude (cf. (6.9)) that

$$
\int_{\alpha}^{\beta} \phi\left(u_{\varepsilon}(t)\right) \mathrm{d} \mu_{\varepsilon}(t) \leq \int_{\alpha}^{\beta} \phi(\gamma(L(t))) \mathrm{d} \mu_{\varepsilon}(t) .
$$


In turn,

$$
\begin{aligned}
& \int_{\alpha}^{\beta} \phi(\gamma(L(t))) \mathrm{d} \mu_{\varepsilon}(t) \\
& \stackrel{(1)}{\leq} \int_{\alpha}^{\beta}(1-\mathrm{L}(\alpha, \beta ; t)) \phi\left(u_{\varepsilon}(\alpha)\right)+\mathrm{L}(\alpha, \beta ; t) \phi\left(u_{\varepsilon}(\beta)\right)-\frac{\lambda}{2}(1-\mathrm{L}(\alpha, \beta ; t)) \mathrm{L}(\alpha, \beta ; t)(L(\beta)-L(\alpha))^{2} \mathrm{~d} \mu_{\varepsilon}(t) \\
& \stackrel{(2)}{=} \int_{\alpha}^{\beta} P(L(t)) \mathrm{d} \mu_{\varepsilon}(t)
\end{aligned}
$$

where (1) follows from the $\lambda$-geodesic convexity of $\phi$ and the fact that $\gamma(L(\alpha))=u_{\varepsilon}(\alpha)$ and $\gamma(L(\beta))=u_{\varepsilon}(\beta)$, while $(2)$ ensues from the fact that $\phi\left(u_{\varepsilon}(\alpha)\right)=P(L(\alpha))$ and $\phi\left(u_{\varepsilon}(\beta)\right)=P(L(\beta))$, cf. (6.28), combined with (6.26). From (6.29) and (6.30) we thus arrive at a contradiction with (6.28). This concludes the proof of (6.25).

The final assertion follows by combining (6.25) with the identity $L^{2}(t)=(1-\mathrm{L}(a, b ; t)) L^{2}(a)+\mathrm{L}(a, b ; t) L^{2}(b)-(1-\mathrm{L}(a, b ; t)) \mathrm{L}(a, b ; t)(L(b)-L(a))^{2} \quad$ for all $t \in[a, b]$, which follows from (6.14).

Corollary 6.9. Assume the LSCC Property 2.5, (6.1) with $\lambda<0$, and let $u_{\varepsilon} \in \mathcal{M}_{\varepsilon}(\bar{u})$. Then, the functions $t \mapsto \phi\left(u_{\varepsilon}(t)\right)$ is continuous on $(0, \infty)$, while $t \mapsto\left|u_{\varepsilon}^{\prime}\right|(t)$ is locally bounded.

Proof. The assertion for $\phi \circ u_{\varepsilon}$ follows from the continuity of the function $t \mapsto \phi\left(u_{\varepsilon}(t)\right)-\frac{\lambda}{2} L^{2}(t)$. Since the mapping $t \mapsto \phi\left(u_{\varepsilon}(t)\right)-\frac{\varepsilon}{2}\left|u_{\varepsilon}^{\prime}\right|^{2}(t)$ is in $W_{\text {loc }}^{1,1}([0, \infty))$, we conclude that $t \mapsto\left|u_{\varepsilon}^{\prime}\right|^{2}(t)$ has a continuous representative, whence the thesis.

We are now in a position to prove inequality (6.5), along with some of the other claims in the statement of Theorem 6.7, in the case $\lambda<0$.

Lemma 6.10. Assume the LSCC Property 2.5, (6.1) with $\lambda<0$, and let $u_{\varepsilon} \in \mathcal{M}_{\varepsilon}(\bar{u})$. Then, $u_{\varepsilon}$ enjoys properties (2), (3) and (5) from the statement of Theorem 6.7. In particular, the function $t \mapsto \phi\left(u_{\varepsilon}(t)\right)$ is locally Lipschitz on $(0, \infty)$.

Proof. Claim 1: For every $[a, b] \subset[0, \infty)$, let $C_{a, b}=\sup _{t \in(a, b)}\left|u_{\varepsilon}^{\prime}\right|^{2}(t)$. Then,

$$
t \mapsto \phi\left(u_{\varepsilon}(t)\right)+\mathcal{U}_{\varepsilon}(t) \text { is } \lambda C_{a, b}-\text { convex on }[a, b] .
$$

Indeed, from inequality (6.8) we deduce that

$$
\begin{aligned}
& \int_{a}^{b}\left(\phi\left(u_{\varepsilon}(t)\right)+\mathcal{U}_{\varepsilon}(t)\right) \mathrm{d} \mu_{\varepsilon}(t) \\
& \leq\left(\phi\left(u_{\varepsilon}(a)\right)+\mathcal{U}_{\varepsilon}(a)\right) \int_{a}^{b}\left(1-\mathrm{I}_{a, b}(t)\right) \mathrm{d} \mu_{\varepsilon}(t)+\left(\phi\left(u_{\varepsilon}(b)\right)+\mathcal{U}_{\varepsilon}(b)\right) \int_{a}^{b} \mathrm{I}_{a, b}(t) \mathrm{d} \mu_{\varepsilon}(t) \\
& \quad-\frac{\lambda C_{a, b}}{2}(b-a)^{2}\left(\int_{a}^{b}\left(1-\mathrm{I}_{a, b}(t)\right) \mathrm{d} \mu_{\varepsilon}(t)\right) \int_{a}^{b} \mathrm{I}_{a, b}(t) \mathrm{d} \mu_{\varepsilon}(t) .
\end{aligned}
$$

We combine this with identity (6.14) to conclude that

$$
\begin{aligned}
& \int_{a}^{b}\left(\phi\left(u_{\varepsilon}(t)\right)+\mathcal{U}_{\varepsilon}(t)-\frac{\lambda C_{a, b}}{2} t^{2}\right) \mathrm{d} \mu_{\varepsilon}(t) \\
& \leq\left(\phi\left(u_{\varepsilon}(a)\right)+\mathcal{U}_{\varepsilon}(a)-\frac{\lambda C_{a, b}}{2} a^{2}\right) \int_{a}^{b}\left(1-\mathrm{I}_{a, b}(t)\right) \mathrm{d} \mu_{\varepsilon}(t)+\left(\phi\left(u_{\varepsilon}(b)\right)+\mathcal{U}_{\varepsilon}(b)-\frac{\lambda C_{a, b}}{2} b^{2}\right) \int_{a}^{b} \mathrm{I}_{a, b}(t) \mathrm{d} \mu_{\varepsilon}(t) .
\end{aligned}
$$

Therefore, applying Lemma 6.5 we conclude that the function $\psi(t):=\phi\left(u_{\varepsilon}(t)\right)+\mathcal{U}_{\varepsilon}(t)-\frac{\lambda C_{a, b}}{2} t^{2}$ is convex, whence the desired (6.31).

Claim 2: there holds

$$
-\varepsilon \frac{\mathrm{d}^{2}}{\mathrm{~d} t^{2}}\left(\frac{1}{2}\left|u_{\varepsilon}^{\prime}\right|^{2}(t)\right)+\frac{\mathrm{d}}{\mathrm{d} t} \frac{1}{2}\left|u_{\varepsilon}^{\prime}\right|^{2}(t)=-\frac{\mathrm{d}^{2}}{\mathrm{~d} t^{2}} \phi\left(u_{\varepsilon}(t)\right)-\frac{\mathrm{d}}{\mathrm{d} t} \frac{1}{2}\left|u_{\varepsilon}^{\prime}\right|^{2}(t) \leq-\lambda C_{a, b} \text { in } \mathcal{D}^{\prime}(a, b) .
$$


It follows from $(6.31)$ that

$$
\frac{\mathrm{d}^{2}}{\mathrm{~d} t^{2}}\left(\phi\left(u_{\varepsilon}(t)\right)+\mathcal{U}_{\varepsilon}(t)\right) \geq \lambda C_{a, b} \quad \text { in } \mathcal{D}^{\prime}(a, b)
$$

and thus, rewriting the metric inner variation equation (3.20) in the form (6.21) and rearranging the terms, we have (6.32).

Claim 3: the function $t \mapsto \frac{1}{2}\left|u_{\varepsilon}^{\prime}\right|^{2}(t)$ admits a locally Lipschitz representative.

We again use the notation $w_{\varepsilon}(t)=\frac{1}{2}\left|u^{\prime}\right|^{2}(t)$. From (6.32) we deduce that $w_{\varepsilon}$ fulfills $-\varepsilon w_{\varepsilon}^{\prime \prime}+w_{\varepsilon}^{\prime} \leq$ $-\lambda C_{a, b}$ in $\mathcal{D}^{\prime}(a, b)$ which, setting $\nu:=-\lambda C_{a, b}$, we rewrite as

$$
-\varepsilon \mathrm{e}^{t / \varepsilon} \frac{\mathrm{d}}{\mathrm{d} t}\left(\mathrm{e}^{-t / \varepsilon}\left(w_{\varepsilon}^{\prime}-\nu\right)\right) \leq 0 \text { in } \mathcal{D}^{\prime}(a, b)
$$

Now, let us set $\mathcal{L}(t)=w_{\varepsilon}(t)-\nu t$. It follows from (6.34) that the distributional derivative of $\mathcal{L}$ is locally bounded, so that $\mathcal{L}$ admits a locally Lipschitz representative, whence the claim for $w_{\varepsilon}$. From now on, we will identify $t \mapsto \frac{1}{2}\left|u_{\varepsilon}^{\prime}\right|^{2}(t)$ with its locally Lipschitz representative.

Claim 4: the function $t \mapsto \phi\left(u_{\varepsilon}(t)\right)$ is locally Lipschitz on $(0, \infty)$. Since the function $t \mapsto$ $\frac{1}{2}\left|u_{\varepsilon}^{\prime}\right|^{2}(t)$ is locally bounded, its primitive $\mathcal{U}_{\varepsilon}$ is locally Lipschitz on $(0, \infty)$. In turn, by Claim 1 the mapping $t \mapsto \phi\left(u_{\varepsilon}(t)\right)+\mathcal{U}_{\varepsilon}(t)$ is locally Lipschitz on $[a, b]$ for every $[a, b] \subset(0, \infty)$. The claim follows.

Claim 5: (6.5) holds. Let $\mathrm{f}$ be the density of the distributional derivative $-\frac{\mathrm{d}^{2}}{\mathrm{~d} t^{2}}\left(\phi\left(u_{\varepsilon}(t)\right)+\mathcal{U}_{\varepsilon}(t)\right)$. It follows from (6.33) that

$$
\mathrm{f}(t) \leq-\lambda \sup _{s \in[a, b]}\left|u_{\varepsilon}^{\prime}\right|^{2}(s) \quad \text { for a.a. } t \in(a, b), \text { for all }[a, b] \subset(0, \infty) .
$$

Let $t \in(0, \infty)$, out of a negligible set, be a Lebesgue point for $\mathrm{f}$. Then,

$$
\mathrm{f}(t)=\lim _{r \downarrow 0} \frac{1}{r} \int_{t}^{t+r} \mathrm{f}(s) \mathrm{d} s \leq \lim _{r \downarrow 0}\left(-\lambda \sup _{s \in[t, t+r]}\left|u_{\varepsilon}^{\prime}\right|^{2}(s)\right)=-\lambda\left|u_{\varepsilon}^{\prime}\right|^{2}(t),
$$

whence (6.5).

This concludes the proof.

We are now in a position to conclude the

Proof of Theorem 6.7. In view of Lemma 6.10, it remains to prove properties (4) and (6), as well the right-continuity of $t \mapsto \phi\left(u_{\varepsilon}(t)\right)$ at $t=0$. We split the proof in several claims.

Claim 1: there exists a family $\left(x_{2}^{\varepsilon}\right)_{\varepsilon} \subset(0, \infty)$ such that $x_{2}^{\varepsilon} \downarrow-2 \lambda$ as $\varepsilon \downarrow 0$ and

$$
\mathrm{e}^{-x_{2}^{\varepsilon} t}\left|u_{\varepsilon}^{\prime}\right|^{2} \text { is nonincreasing. }
$$

Then, (1) in Theorem 6.7 follows upon choosing, for every prescribed $\lambda^{\prime}<\lambda, \varepsilon^{\prime}>0$ such that for all $\varepsilon \in\left(0, \varepsilon^{\prime}\right)$ there holds with $-\frac{1}{2} x_{2}^{\varepsilon}>\lambda^{\prime}$.

We combine (6.5) with the metric inner variation equation, cf. (6.21), and deduce that (cf. (6.22))

$$
-\varepsilon w_{\varepsilon}^{\prime \prime}+w_{\varepsilon}^{\prime}+2 \lambda w_{\varepsilon} \leq 0 \quad \text { in } \mathcal{D}^{\prime}(0, \infty),
$$

where again we have used the place-holder $w_{\varepsilon}:=\frac{1}{2}\left|u_{\varepsilon}^{\prime}\right|^{2}$. Let us introduce the negative distributions $\nu_{\varepsilon}$ and $h_{\varepsilon}$ by

$$
h_{\varepsilon}:=\frac{\nu_{\varepsilon}}{\varepsilon} \quad \text { and } \nu_{\varepsilon}:=-\varepsilon w_{\varepsilon}^{\prime \prime}+w_{\varepsilon}^{\prime}+2 \lambda w_{\varepsilon} .
$$

Hence,

$$
w_{\varepsilon}^{\prime \prime}-\frac{1}{\varepsilon} w_{\varepsilon}^{\prime}-\frac{2 \lambda}{\varepsilon} w_{\varepsilon}=-h_{\varepsilon} \quad \text { in } \mathcal{D}^{\prime}(0, \infty) .
$$

The general solution of (6.37) has the form

$$
w_{\varepsilon}(t)=A \mathrm{e}^{x_{1}^{\varepsilon} t}+B \mathrm{e}^{x_{2}^{\varepsilon} t}+\int_{-\infty}^{\infty} E(t-s)\left(-h_{\varepsilon}(s)\right) \mathrm{d} s,
$$


where $x_{1}^{\varepsilon}$ and $x_{2}^{\varepsilon}$ are the two (real) roots of the characteristic equation and $E$ is the fundamental solution with support in $(-\infty, 0]$. We have that

$$
x_{1}^{\varepsilon}=\frac{1+\sqrt{1+8 \lambda \varepsilon}}{2 \varepsilon}, \quad x_{2}^{\varepsilon}=\frac{1-\sqrt{1+8 \lambda \varepsilon}}{2 \varepsilon} .
$$

Note that (at least for sufficiently small $\varepsilon$ ) $x_{1}^{\varepsilon}$ and $x_{2}^{\varepsilon}$ are positive. Consequently,

$$
w_{\varepsilon}(t)=\int_{-\infty}^{\infty} E(t-s)\left(-h_{\varepsilon}(s)\right) \mathrm{d} s,
$$

since $w_{\varepsilon}$ must be integrable on $(0, \infty)$. The function $E$ is the fundamental solution with support in $(-\infty, 0)$ and can be found by solving the following Cauchy problem

$$
\left\{\begin{array}{l}
v^{\prime \prime}(t)-\frac{1}{\varepsilon} v^{\prime}(t)-\frac{2 \lambda}{\varepsilon} v(t)=0, \\
v(0)=0 \\
v^{\prime}(0)=-1 .
\end{array}\right.
$$

Denoting with $H(\cdot)$ the Heaviside function, we have that

$$
E(t)=-\frac{1}{\theta} \mathrm{e}^{t /(2 \varepsilon)} \sinh (\theta(t)) H(-t) \quad \text { with the place-holder } \theta:=\frac{\sqrt{1+8 \lambda \varepsilon}}{2 \varepsilon} .
$$

Therefore, from (6.39) we gather that $w_{\varepsilon}(t)=\frac{1}{\theta} \int_{t}^{\infty} h_{\varepsilon}(s) \mathrm{e}^{(t-s) /(2 \varepsilon)} \sinh (\theta(t-s)) \mathrm{d} s$, which we rewrite as

$$
w_{\varepsilon}(t)=\frac{\mathrm{e}^{x_{2}^{\varepsilon} t}}{\theta} \int_{t}^{\infty} h_{\varepsilon}(s) \mathrm{e}^{-s / 2 \varepsilon} f(t, s) \mathrm{d} s
$$

where $f t, s):=\frac{1}{2}\left(\mathrm{e}^{2 \theta t} \mathrm{e}^{-\theta s}-\mathrm{e}^{\theta s}\right)$. Now, differentiating with respect to $t$ we find

$$
w_{\varepsilon}^{\prime}(t)=x_{2}^{\varepsilon} w_{\varepsilon}(t)+\frac{\mathrm{e}^{x_{2}^{\varepsilon} t}}{\theta}\left(-h_{\varepsilon}(t) \mathrm{e}^{-t / 2 \varepsilon} f(t, t)+\int_{t}^{\infty} h_{\varepsilon}(s) \mathrm{e}^{-s / 2 \varepsilon} \partial_{t} f(t, s) \mathrm{d} s\right) .
$$

Thus,

$$
w_{\varepsilon}^{\prime}(t) \leq x_{2}^{\varepsilon} w_{\varepsilon}(t) \quad \text { in } \mathcal{D}^{\prime}(0, \infty)
$$

since $f(t, t)=0$ and $h_{\varepsilon} \leq 0$ by construction while, in turn, $\partial_{t} f(t, s) \geq 0$. As a consequence, we have

whence (6.35).

$$
\frac{\mathrm{d}}{\mathrm{d} t}\left(\mathrm{e}^{-x_{2}^{\varepsilon} t} w_{\varepsilon}(t)\right) \leq 0 \quad \text { in } \mathcal{D}^{\prime}(0, \infty)
$$

Claim 2: the function $\phi \circ u_{\varepsilon}$ is nonincreasing.

Indeed, from (6.21) we gather that

$$
\frac{\mathrm{d}}{\mathrm{d} t}\left(\phi \circ u_{\varepsilon}\right)=\varepsilon w_{\varepsilon}^{\prime}-2 w_{\varepsilon} \leq\left(\varepsilon x_{2}^{\varepsilon}-2\right) w_{\varepsilon} \leq 0 \quad \text { in } \mathcal{D}^{\prime}(0, \infty)
$$

where the first inequality holds in view of (6.43). The second one is true for a sufficiently small $\varepsilon$, since $x_{2}^{\varepsilon}$ converges to $-2 \lambda$.

Finally, The continuity of $t \mapsto \phi\left(u_{\varepsilon}(t)\right)$ at $t=0$ follows from its previously proved monotonicity, arguing in the very same way as in the proof of Thm. 6.4, cf. (6.24).

This concludes the proof of Thm. 6.7.

\section{The metric Hamilton-JAcobi equation and the gradient flow of $V_{\varepsilon}$}

In this section we get further insight into the interpretation of WED minimizers as curves of maximal slope with respect to $V_{\varepsilon}$. Our starting point will again be the fundamental identity (4.11) satisfied by any WED minimizer $u_{\varepsilon}$, viz.

$$
-\frac{\mathrm{d}}{\mathrm{d} t} V_{\varepsilon}\left(u_{\varepsilon}(t)\right)=\frac{1}{2}\left|u_{\varepsilon}^{\prime}\right|^{2}(t)+\frac{1}{\varepsilon} \phi\left(u_{\varepsilon}(t)\right)-\frac{1}{\varepsilon} V_{\varepsilon}\left(u_{\varepsilon}(t)\right) \quad \text { for a.a. } t \in(0, \infty),
$$

but we shall adopt a different viewpoint in comparison to Theorem 4.6. Indeed, here we will combine (4.11) with the metric analogue of identity (1.13), which in turn derived from the HamiltonJacobi equation (1.12). That is why, we may refer to (7.1) below, relating the functional $\frac{1}{\varepsilon}\left(\phi-V_{\varepsilon}\right)$ 
with (a suitable version of ) the local slope of $V_{\varepsilon}$, cf. (7.1) ahead, as a (metric) Hamilton-Jacobi identity. Let us mention in advance that the proof of (7.1) relies on the $\lambda$-geodesic convexity of $\phi$, for some $\lambda \in \mathbb{R}$. From this we will deduce in Corollary 7.4 that $u_{\varepsilon}$ is a curve of maximal slope for $V_{\varepsilon}$, albeit with respect to this suitably modified notion of slope.

We set

$$
\left|\tilde{\partial} V_{\varepsilon}\right|(u):=\limsup _{v \rightarrow u, \phi(v) \rightarrow \phi(u)} \frac{\left(V_{\varepsilon}(u)-V_{\varepsilon}(v)\right)^{+}}{\mathrm{d}(u, v)} \quad \text { for } u \in X
$$

and refer to $\left|\tilde{\partial} V_{\varepsilon}\right|$ as the $\phi$-conditioned (local) slope of $V_{\varepsilon}$ at $u$, to highlight that, in its definition we restrict to sequences converging to $u$ with converging $\phi$-energy. Clearly, we have

$$
\left|\tilde{\partial} V_{\varepsilon}\right|(u) \leq\left|\partial V_{\varepsilon}\right|(u) \text { for all } u \in X
$$

With the main result of this section we establish the Hamilton-Jacobi identity for the value functional.

Theorem 7.1. Under the LSCC Property 2.5 and (6.1) for some $\lambda \in \mathbb{R}$, there holds

$$
\sqrt{2 \frac{\phi(u)-V_{\varepsilon}(u)}{\varepsilon}}=G_{\varepsilon}(u)=\left|\tilde{\partial} V_{\varepsilon}\right|(u) \quad \text { for all } u \in \mathrm{D}(\phi) .
$$

We split the proof in the two following lemmas.

Lemma 7.2. Under the LSCC Property 2.5 and (6.1), there holds

$$
G_{\varepsilon}(\bar{u}) \leq\left|\tilde{\partial} V_{\varepsilon}\right|(\bar{u}) \quad \text { for all } \bar{u} \in \mathrm{D}(\phi)
$$

holds.

Proof. Let $u_{\varepsilon} \in \mathcal{M}_{\varepsilon}(\bar{u})$ and $\delta>0$. We have

$$
\begin{gathered}
\frac{\left(V_{\varepsilon}(u)-V_{\varepsilon}\left(u_{\varepsilon}(\delta)\right)\right)^{+}}{\mathrm{d}\left(u_{\varepsilon}(\delta), u\right)} \geq \frac{\left(V_{\varepsilon}(u)-V_{\varepsilon}\left(u_{\varepsilon}(\delta)\right)\right)^{+}}{\int_{0}^{\delta}\left|u_{\varepsilon}^{\prime}\right|(s) \mathrm{d} s} \geq \frac{\left(V_{\varepsilon}(u)-V_{\varepsilon}\left(u_{\varepsilon}(\delta)\right)\right)^{+}}{\delta^{1 / 2}\left(\int_{0}^{\delta}\left|u_{\varepsilon}^{\prime}\right|^{2}(s) \mathrm{d} s\right)^{1 / 2}} \\
=\frac{\int_{0}^{\delta} G_{\varepsilon}^{2}\left(u_{\varepsilon}(s)\right) \mathrm{d} s}{\delta^{1 / 2}\left(\int_{0}^{\delta} G_{\varepsilon}^{2}\left(u_{\varepsilon}(s)\right) \mathrm{d} s\right)^{1 / 2}},
\end{gathered}
$$

where the latter identity follows from the fact that $u_{\varepsilon}$ is a curve of maximal slope for $V_{\varepsilon}$ w.r.t. $G_{\varepsilon}$, cf. Corollary 4.7 , so that $\left|u_{\varepsilon}^{\prime}\right|=G_{\varepsilon}\left(u_{\varepsilon}\right)$ a.e. in $(0, \infty)$. Therefore,

$$
\begin{aligned}
G_{\varepsilon}(u) \stackrel{(1)}{\leq} \liminf _{\delta \rightarrow 0}\left(\frac{1}{\delta} \int_{0}^{\delta} G_{\varepsilon}^{2}\left(u_{\varepsilon}(s)\right) \mathrm{d} s\right)^{1 / 2} & \leq \limsup _{\delta \rightarrow 0} \frac{\left(V_{\varepsilon}(u)-V_{\varepsilon}\left(u_{\varepsilon}(\delta)\right)\right)^{+}}{\mathrm{d}\left(u_{\varepsilon}(\delta), u\right)} \\
& \stackrel{(2)}{\leq} \limsup _{v \rightarrow u \phi(v) \rightarrow \phi(u)} \frac{\left(V_{\varepsilon}(u)-V_{\varepsilon}(v)\right)^{+}}{\mathrm{d}(v, u)}=\left|\tilde{\partial} V_{\varepsilon}\right|(u) .
\end{aligned}
$$

Indeed, (1) follows from the lower semicontinuity of the mapping $t \mapsto G_{\varepsilon}^{2}\left(u_{\varepsilon}(t)\right)$ on $[0, \infty)$, which is in turn guaranteed by the lower semicontinuity of $t \mapsto \phi\left(u_{\varepsilon}(t)\right)$ and the continuity of $t \mapsto V_{\varepsilon}\left(u_{\varepsilon}(t)\right)$ thanks to Lemma 6.1: the latter result applies since $\sup _{t \in[0, \infty)} \phi\left(u_{\varepsilon}(t)\right) \leq \phi(\bar{u})$ by Theorems 6.4 and 6.7. Further, (2) is due to the fact that $\phi\left(u_{\varepsilon}(\delta)\right) \rightarrow \phi(u)$ as $\delta \rightarrow 0$, since $\phi \circ u_{\varepsilon}$ is rightcontinuous at $t=0$ (cf. Theorems 6.4 and 6.7 ).

In fact, in the proof of Lemma 7.2 the $\lambda$-geodesic convexity (6.1) has been used only in that it guarantees that the map $t \mapsto \phi\left(u_{\varepsilon}(t)\right)$ is bounded on $[0, \infty)$ and right-continuous at $t=0$. Instead, in the proof of Lemma 7.3 below, (6.1) is used more explicitly.

Lemma 7.3. Under Property 2.5 and (6.1), there holds $\mathrm{D}(\phi) \subset \mathrm{D}\left(\left|\tilde{\partial} V_{\varepsilon}\right|\right)$ and

$$
G_{\varepsilon}(u) \geq\left|\tilde{\partial} V_{\varepsilon}\right|(u) \quad \text { for all } u \in \mathrm{D}(\phi) .
$$


Proof. Let us fix $v \in \mathrm{D}(\phi)$ and fix $r>0$. We set $\delta=\frac{\mathrm{d}(u, v)}{r}$. Let us denote by $\gamma$ the constant-speed geodesic

$$
\gamma:[0, \delta] \rightarrow X \text { such that } \gamma(0)=u, \gamma(\delta)=v, \quad\left|\gamma^{\prime}\right|(t)=\frac{\mathrm{d}(u, v)}{\delta}=r \quad \text { for a.a. } t \in(0, \delta) .
$$

Furthermore, let $v_{\varepsilon} \in \mathcal{M}_{\varepsilon}(v)$ and, finally, let us consider the curve $\zeta:[\delta, \infty) \rightarrow X$ given by $\zeta(t)=v_{\varepsilon}(t-\delta)$. Hence $\zeta(\delta)=v_{\varepsilon}(0)=v$, and the curve $\tilde{u}:[0, \infty) \rightarrow X$ defined by

$$
\tilde{u}(t)= \begin{cases}\gamma(t) & t \in[0, \delta] \\ \zeta(t) & t \in[\delta, \infty)\end{cases}
$$

is absolutely continuous, fulfils $\tilde{u}(0)=u$, and can thus be chosen as a competitor in the minimum problem which defines $V_{\varepsilon}$. Hence,

$$
\begin{aligned}
V_{\varepsilon}(u) & \leq \int_{0}^{\infty} \ell_{\varepsilon}\left(t, \tilde{u}(t),\left|\tilde{u}^{\prime}\right|(t)\right) \mathrm{d} t \\
& =\int_{0}^{\delta} \ell_{\varepsilon}\left(t, \gamma(t),\left|\gamma^{\prime}\right|(t)\right) \mathrm{d} t+\int_{\delta}^{\infty} \ell_{\varepsilon}\left(t, \zeta(t),\left|\zeta^{\prime}\right|(t)\right) \mathrm{d} t \\
& =\int_{0}^{\delta} \ell_{\varepsilon}\left(t, \gamma(t),\left|\gamma^{\prime}\right|(t)\right) \mathrm{d} t+\mathrm{e}^{-\delta / \varepsilon} \int_{0}^{\infty} \ell_{\varepsilon}\left(t, v_{\varepsilon}(t),\left|v_{\varepsilon}^{\prime}\right|(t)\right) \mathrm{d} t,
\end{aligned}
$$

where the last integral equals $V_{\varepsilon}(v)$. Therefore,

$$
V_{\varepsilon}(u)-\mathrm{e}^{-\delta / \varepsilon} V_{\varepsilon}(v) \leq \int_{0}^{\delta} \mathrm{e}^{-t / \varepsilon}\left(\frac{1}{2} \frac{\mathrm{d}^{2}(u, v)}{\delta^{2}}+\frac{1}{\varepsilon} \phi(\gamma(t))\right) \mathrm{d} t
$$

Using that $\phi$ is $\lambda$-geodesically-convex, we conclude that

$$
\begin{aligned}
V_{\varepsilon}(u)-\mathrm{e}^{-\delta / \varepsilon} V_{\varepsilon}(v) \leq & \frac{1}{2} \frac{\mathrm{d}^{2}(u, v)}{\delta^{2}} \int_{0}^{\delta} \mathrm{e}^{-t / \varepsilon} \mathrm{d} t+\frac{1}{\varepsilon} \max \{\phi(u), \phi(v)\} \int_{0}^{\delta} \mathrm{e}^{-t / \varepsilon} \mathrm{d} t \\
& -\frac{\lambda \mathrm{d}^{2}(u, v)}{2 \delta^{2}} \int_{0}^{\delta} \mathrm{e}^{-t / \varepsilon}(\delta-t) t \mathrm{~d} t \\
= & \varepsilon\left(1-\mathrm{e}^{-\delta / \varepsilon}\right)\left(\frac{\mathrm{d}^{2}(u, v)}{2 \delta^{2}}+\frac{1}{\varepsilon} \max \{\phi(u), \phi(v)\}\right)-\frac{\lambda \mathrm{d}^{2}(u, v)}{2 \delta^{2}} \int_{0}^{\delta} \mathrm{e}^{-t / \varepsilon}(\delta-t) t \mathrm{~d} t .
\end{aligned}
$$

We now add to both sides of the equality the term $V_{\varepsilon}(v)$, and divide by $\mathrm{d}(u, v)$, thus obtaining

$$
\begin{aligned}
& \frac{V_{\varepsilon}(u)-V_{\varepsilon}(v)}{\mathrm{d}(u, v)} \\
& \leq \frac{1}{2} \frac{\mathrm{d}^{2}(u, v)}{\delta^{2}} \frac{\delta}{\mathrm{d}(u, v)} \frac{1-\mathrm{e}^{-\delta / \varepsilon}}{\frac{\delta}{\varepsilon}}+\frac{\delta}{\mathrm{d}(u, v)} \frac{1-\mathrm{e}^{-\delta / \varepsilon}}{\delta} \max \{\phi(u), \phi(v)\} \\
& \quad-\frac{\delta}{\mathrm{d}(u, v)} \frac{1-\mathrm{e}^{-\delta / \varepsilon}}{\delta} V_{\varepsilon}(v)-\frac{\lambda \mathrm{d}^{2}(u, v)}{2 \delta^{2}} \int_{0}^{\delta} \mathrm{e}^{-t / \varepsilon}(\delta-t) t \mathrm{~d} t=: \Lambda_{1}+\Lambda_{2}+\Lambda_{3}+\Lambda_{4} .
\end{aligned}
$$

Then, we take the $\lim \sup$ as $v \rightarrow u$, with $\phi(v) \rightarrow \phi(u)$, of the above inequality. Notice that, we may suppose that $V_{\varepsilon}(v) \leq V_{\varepsilon}(u)$. As $v \rightarrow u$, we have that $\delta \rightarrow 0$, and

$$
\begin{aligned}
\limsup _{v \rightarrow u}\left(\Lambda_{1}\right) & \leq \frac{1}{2} r \\
\limsup _{v \rightarrow u}\left(\Lambda_{2}\right) & \leq \frac{1}{\varepsilon} \frac{1}{r} \phi(u) \\
\limsup _{v \rightarrow u}\left(\Lambda_{3}\right) & =-\liminf _{v \rightarrow u} \frac{\delta}{\mathrm{d}(u, v)} \frac{1-\mathrm{e}^{-\delta / \varepsilon}}{\delta} V(v) \leq-\frac{1}{\varepsilon} \frac{1}{r} V(u),
\end{aligned}
$$

where the second limit follows from the fact that $\phi(v) \rightarrow \phi(u)$, and for the third limit we have used that $V_{\varepsilon}$ is lower semicontinuous. We also have $\lim \sup _{v \rightarrow u}\left(\Lambda_{4}\right)=0$. In conclusion, we find $\left|\tilde{\partial} V_{\varepsilon}\right|(u)=\limsup _{v \rightarrow u, \phi(v) \rightarrow \phi(u), V_{\varepsilon}(v) \leq V_{\varepsilon}(u)} \frac{V_{\varepsilon}(u)-V_{\varepsilon}(v)}{\mathrm{d}(u, v)} \leq \frac{1}{2} r+\frac{1}{r}\left(\frac{1}{\varepsilon} \phi(u)-\frac{1}{\varepsilon} V_{\varepsilon}(u)\right) \quad$ for all $r>0$. 
Therefore,

$$
\frac{1}{2}\left|\tilde{\partial} V_{\varepsilon}\right|^{2}(u)=\sup _{r>0}\left(\left|\tilde{\partial} V_{\varepsilon}\right|(u) r-\frac{1}{2} r^{2}\right) \leq \frac{1}{\varepsilon} \phi(u)-\frac{1}{\varepsilon} V_{\varepsilon}(u),
$$

whence (7.3).

As a straightforward consequence of the (metric) Hamilton-Jacobi equation (7.1) and of Corollary 4.7 , we have

Corollary 7.4. Assume Property 2.5 and (6.1). Then, for every fixed $\varepsilon>0$ the curve $u_{\varepsilon}$ fulfils

$$
\frac{\mathrm{d}}{\mathrm{d} t} V_{\varepsilon}\left(u_{\varepsilon}(t)\right)=-\frac{1}{2}\left|u_{\varepsilon}^{\prime}\right|^{2}(t)-\frac{1}{2}\left|\tilde{\partial} V_{\varepsilon}\right|^{2}\left(u_{\varepsilon}(t)\right) \quad \text { for a.a. } t \in(0, T),
$$

i.e. $u_{\varepsilon}$ is a curve of maximal slope for $V_{\varepsilon}$, with respect to the ( $L^{1}$-moderated) upper gradient $\left|\tilde{\partial} V_{\varepsilon}\right|$.

We conclude this Section getting further insight into the relationship between $\left|\tilde{\partial} V_{\varepsilon}\right|$ and $|\partial \phi|$. The following result is an immediate corollary of Prop. 5.3, Cor. 5.5, and Thm. 7.1.

Corollary 7.5. Assume Property 2.5 and (6.1). Then, for all $u \in \mathrm{D}(\phi)$

$$
\left|\partial^{-} \phi\right|(u) \leq \liminf _{\varepsilon \downarrow 0}\left|\tilde{\partial} V_{\varepsilon}\right|(u) \leq \limsup _{\varepsilon \downarrow 0}\left|\tilde{\partial} V_{\varepsilon}\right|(u) \leq|\partial \phi|(u) .
$$

\section{Applications}

The aim of this section is to present some application of the abstract theory. In particular, we comment on the framing of our main result Theorem 3.6 in two different variational settings, namely in Banach spaces (Sec. 8.1) and in Wasserstein spaces of probability measures (Sec. 8.2).

8.1. Application to gradient flows in Banach spaces. We take as ambient space $X$ a reflexive and separable Banach space $\mathcal{B}$, with norm $\|\cdot\|$ and corresponding duality mapping $J: \mathcal{B} \rightrightarrows \mathcal{B}^{*}$, defined by

$$
\xi \in J(v) \text { if and only if }\langle\xi, v\rangle_{\mathcal{B}}=\|v\|^{2}=\|\xi\|_{*}^{2} .
$$

Given an energy functional $\phi: \mathcal{B} \rightarrow(-\infty, \infty]$ we are interested in trajectories $u:[0, \infty) \rightarrow \mathrm{D}(\phi)$ solving

$$
J\left(u^{\prime}(t)\right)+\partial^{\circ} \phi(u(t)) \ni 0 \quad \text { in } \mathcal{B}^{*} \quad \text { for a.a. } t \in(0, \infty) .
$$

Here, $\partial^{\circ} \phi$ denotes the sets of elements of minimal norm (the minimal section) in the Fréchet subdifferential of $\phi$, defined at $u \in D(\phi)$ by

$$
\xi \in \partial \phi(u) \text { if and only if } \phi(v)-\phi(u) \geq\langle\xi, v-u\rangle_{\mathcal{B}}+o(\|v-u\|) \text { as } v \rightarrow u,
$$

so that $\partial \phi$ coincides with the subdifferential of convex analysis of $\phi$ (and is thus denoted by the same symbol) as soon as $\phi$ is convex. Since for every $u \in D(\partial \phi)$ the set $\partial \phi(u)$ is convex and weakly*-closed in $\mathcal{B}^{*}, \partial^{\circ} \phi(u)$ is well defined and satisfies

$$
|\partial \phi|(u) \leq\|\xi\|_{*} \quad \forall \xi \in \partial \phi(u) .
$$

The next result (see [AGS08]) provides a connection between (8.2) and curves of maximal slope.

Proposition 8.1. Assume Property 2.5 with respect to the strong topology of $\mathcal{B}$ and suppose that the graph of the Fréchet subdifferential of $\phi$ is strongly-weakly closed, i.e.

$$
\left.\begin{array}{l}
u_{n} \in \mathcal{B}, \xi_{n} \in \mathcal{B}^{*} \text { with } \xi_{n} \in \partial \phi\left(u_{n}\right) \text { for all } n \in \mathbb{N} \\
u_{n} \rightarrow u, \xi_{n} \rightarrow \xi \text { as } n \rightarrow \infty, \sup _{n} \phi\left(u_{n}\right)<\infty
\end{array}\right\} \Rightarrow \xi \in \partial \phi(u) \text {. }
$$

There holds

$$
\left|\partial^{-} \phi\right|(u)=|\partial \phi|(u)=\|\xi\|_{*} \quad \text { for every } \xi \in \partial^{\circ} \phi(u)
$$

Furthermore, if $|\partial \phi|$ is a $L^{\infty}$-moderated upper gradient for $\phi$, then $u$ is a curve of maximal slope w.r.t. $|\partial \phi|$ if and only if it is a solution of the gradient flow (8.2). 
Functionals of the Calculus of Variations. In our abstract framework we can consider the socalled Functionals of the Calculus of Variations, cf. e.g. [Lio69, Chap. 2.5]. We limit our analysis to one of the simplest examples, in the Banach space $\mathcal{B}=L^{\alpha}(\Omega), 1<\alpha<\infty$, where $\Omega$ is a bounded Lipschitz open subset of $\mathbb{R}^{d}$. We consider a Carathéodory integrand $f: \Omega \times \mathbb{R}^{d} \times \mathbb{R} \rightarrow \mathbb{R}$ $f(x, \boldsymbol{z}, u)$, which is strictly convex with respect to $\boldsymbol{z}$ for every $x \in \Omega$ and $u \in \mathbb{R}$, of class $\mathrm{C}^{1}$ w.r.t. $(\boldsymbol{z}, u)$ for a.e. $x \in \Omega$, and satisfies the following coercivity and growth conditions for some $p \in[\alpha, \infty)$ (for simplicity), with $q=p(1-1 / \alpha)$ and suitable positive constants $M_{i}$ :

$$
\left\{\begin{array}{l}
f(x, \boldsymbol{z}, u) \geq M_{1}|\boldsymbol{z}|^{p}-M_{2} \\
\left|\nabla_{\boldsymbol{z}} f(x, \boldsymbol{z}, u)\right| \leq M_{3}\left(1+|\boldsymbol{z}|^{p-1}+|u|^{p-1}\right) \\
\left|f_{u}(x, \boldsymbol{z}, u)\right| \leq M_{4}\left(1+|\boldsymbol{z}|^{q}+|u|^{q}\right)
\end{array} \quad \forall(x, \boldsymbol{z}, u) \in \Omega \times \mathbb{R}^{d} \times \mathbb{R}\right.
$$

(with $f_{u}$ the derivative of $f$ w.r.t. $u$ and $\nabla_{\boldsymbol{z}} f$ its gradient w.r.t. $\boldsymbol{z}$ ). We consider the integral functional $\phi: L^{\alpha}(\Omega) \rightarrow(-\infty, \infty]$ defined via

$$
\phi(u):= \begin{cases}\int_{\Omega} f(x, \nabla u(x), u(x)) \mathrm{d} x & \text { if } u \in W_{0}^{1, p}(\Omega), \\ \infty & \text { otherwise. }\end{cases}
$$

Lemma 8.2. The Fréchet subdifferential $\partial \phi(u)$ of $\phi$, with respect to the topology of $\mathcal{B}=L^{\alpha}(\Omega)$, is non-empty if and only if $A(u):=-\operatorname{div}\left(\nabla_{\boldsymbol{z}} f(\cdot, \nabla u, u)\right)+f_{u}(\cdot, \nabla u, u) \in L^{\alpha^{\prime}}(\Omega)$, and in that case it is given by

$$
\partial \phi(u)=\{A(u)\}
$$

Furthermore, $\partial \phi$ is strongly/weakly closed in $L^{\alpha}(\Omega) \times L^{\alpha^{\prime}}(\Omega)$ along sequences with bounded energy, and $|\partial \phi|$ is an $L^{\infty}$-moderated upper gradient for $\phi$.

Proof. In order to clarify the calculations, let us denote by $V$ the Banach space $W_{0}^{1, p}(\Omega)$; we have $V \hookrightarrow \mathcal{B}$ and $\mathcal{B}^{\prime} \hookrightarrow V^{\prime}$ with compact inclusions.

Let us first notice that the restriction of the functional $\phi$ to $V$ is continuous; moreover, due to the strict convexity with respect to the gradient variable and to the coercivity assumption, if $u_{n} \rightarrow u$ in $V$ and $\phi\left(u_{n}\right) \rightarrow \phi(u)$ we deduce that $u_{n} \rightarrow u$ strongly in $V$.

We consider the functional $\Phi: V \times V \rightarrow \mathbb{R}$

$$
\Phi(v, u):=\int_{\Omega} f(x, \nabla v, u) \mathrm{d} x \quad \text { with } \quad \phi(u)=\Phi(u, u),
$$

and the corresponding continuous operators $A_{1}: V \times V \rightarrow V^{\prime}, A_{2}: V \times V \rightarrow \mathcal{B}^{\prime}$

$$
A_{1}(v, u):=-\operatorname{div}\left(\nabla_{\boldsymbol{z}} f(x, \nabla v, u)\right), \quad A_{2}(v, u):=f_{u}(x, \nabla v, u), \quad A(u)=A_{1}(u, u)+A_{2}(u, u) .
$$

By taking directional derivatives, it is immediate to check that $\partial \phi(u)$ may contain just the element $A(u)$. Let us first check that if $A(u) \in \mathcal{B}^{\prime}$ then it is the Fréchet subdifferential of $\phi$. Notice that by the convexity of $\Phi$ with respect to its first variable

$$
\begin{aligned}
\phi(v)-\phi(u) & -\langle A(u), v-u\rangle=\Phi(v, v)-\Phi(u, u)-\langle A(u), v-u\rangle \\
& =\Phi(v, u)-\Phi(u, u)-\left\langle A_{1}(u, u), v-u\right\rangle+\Phi(v, v)-\Phi(v, u)-\left\langle A_{2}(u, u), v-u\right\rangle \\
& \geq \int_{0}^{1} \int_{\Omega}\left(A_{2}(v, u+\lambda(v-u))-A_{2}(u, u)\right)(v-u) \mathrm{d} x \mathrm{~d} \lambda \\
& \geq-\|v-u\|_{L^{\alpha}} \sigma(v, u), \quad \sigma(v, u):=\int_{0}^{1}\left\|A_{2}(v, u+\lambda(v-u))-A_{2}(u, u)\right\|_{L^{\alpha^{\prime}}} \mathrm{d} \lambda
\end{aligned}
$$

In order to check that $A$ is the Fréchet subdifferential of $\phi$ it is not restrictive to assume that $v \rightarrow u$ in $\mathcal{B}$ and $\phi(v) \rightarrow \phi(u)$, so that $v \rightarrow u$ in $V$. We then obtain that $\sigma(v, u) \rightarrow 0$ since $A_{2}$ is continuous from $V \times V$ to $L^{\alpha^{\prime}}(\Omega)$.

The closedness property (8.5) is a consequence of the fact that the operator $A$ is pseudomonotone from $V$ to $V^{\prime}$ [Lio69, Prop. 2.6] and that the inclusion $L^{\alpha^{\prime}}(\Omega) \hookrightarrow W^{-1, p^{\prime}}(\Omega)$ is compact.

Finally, in order to check that $|\partial \phi|$ (which can now be identified with $\|A(\cdot)\|_{L^{\alpha^{\prime}}}$ ) is an $L^{\infty}$ moderated upper gradient, it is sufficient to observe that the quantity $\sigma$ defined in the previous 
calculations is uniformly bounded on the sublevels of $\phi$. On each sublevel there exists a constant $C>0$ such that for every choice of $v, u$ in the sublevel there holds

$$
\phi(v)-\phi(u) \geq-(|\partial \phi|(u)+C)\|v-u\|_{\mathcal{B}}
$$

We can then argue as in [AGS08, Thm. 1.2.5, Lemma 1.2.6].

Lemma 8.1 allows us to apply Proposition 8.1 and Lemma 8.2 and obtain that, given $u_{0} \in$ $L^{\alpha}(\Omega)$, the WED minimizers converge to a solution $u \in H_{\mathrm{loc}}^{1}\left([0, \infty) ; L^{\alpha}(\Omega)\right)$ of

$$
\begin{cases}J_{\alpha}\left(\partial_{t} u\right)-\operatorname{div}\left(\nabla_{\boldsymbol{z}} f(x, \nabla u, u)\right)+f_{u}(x, \nabla u, u)=0 & \text { in } \Omega \times(0, \infty), \\ u=0 & \text { on } \partial \Omega \times(0, \infty), \\ u(\cdot, 0)=u_{0}(\cdot) & \text { in } \Omega,\end{cases}
$$

where $J_{\alpha}: L^{\alpha}(\Omega) \rightarrow L^{\alpha^{\prime}}(\Omega)$ is the duality mapping $J_{\alpha}(v)=\|v\|_{L^{\alpha}}^{2-\alpha}|v|^{\alpha-2} v$.

It is interesting to compare this result with those obtained in [BDM14] for an energy of the type of $\phi$ in $L^{2}(\Omega)$. In [BDM14] the convexity of $f$ is assumed. Our approach allows for some nonconvexity of $f$ and for doubly nonlinear equations, at the price of imposing suitable regularity, coercivity and growth conditions. Of course, when $\alpha=2$, and there exists $-\lambda \geq 0$ such that $(\boldsymbol{z}, u) \mapsto f(x, \boldsymbol{z}, u)-\frac{\lambda}{2} u^{2}$ is convex, growth conditions could be avoided also in our setting since then the functional $\phi$ would be $\lambda$-convex in $L^{2}(\Omega)$.

Limiting subdifferential. When the Fréchet subdifferential does not satisfy the closedness property (8.5), one can consider a relaxed formulation of (8.2) obtained by substituting $\partial \phi$ with the limiting subdifferential of $\phi$, cf., e.g., [Mor06]. At $u \in \mathrm{D}(\phi)$ the limiting subdifferential $\partial_{\ell} \phi(u)$ is defined by

$$
\xi \in \partial_{\ell} \phi(u) \Leftrightarrow\left\{\begin{array}{l}
\exists u_{n} \in \mathcal{B}, \xi_{n} \in \mathcal{B}^{*} \text { with } \xi_{n} \in \partial \phi\left(u_{n}\right) \text { for all } n \in \mathbb{N}, \\
u_{n} \rightarrow u, \xi_{n} \rightarrow \xi \text { as } n \rightarrow \infty, \quad \sup _{n} \phi\left(u_{n}\right)<\infty .
\end{array}\right.
$$

Since $\partial_{\ell} \phi(u)$ is not necessarily weakly* closed, we introduce the following notions

$$
\begin{aligned}
\bar{\partial}_{\ell} \phi(u) & :=\text { weak }^{*} \text { closure of } \partial_{\ell} \phi(u), \\
\left\|\partial_{\ell}^{\circ} \phi\right\|_{*}(u) & :=\min \left\{\|\xi\|_{*}: \xi \in \bar{\partial}_{\ell} \phi(u)\right\}, \\
\partial_{\ell}^{\circ} \phi(u) & :=\left\{\xi \in \bar{\partial}_{\ell} \phi(u), \quad\|\xi\|_{*}=\left\|\partial_{\ell}^{\circ} \phi\right\|_{*}(u)\right\},
\end{aligned}
$$

and we are interested in trajectories $u:[0, \infty) \rightarrow \mathrm{D}(\phi)$ solving

$$
J\left(u^{\prime}(t)\right)+\partial_{\ell}^{\circ} \phi(u(t)) \ni 0 \quad \text { in } \mathcal{B}^{*} \quad \text { for a.a. } t \in(0, T) .
$$

for a functional $\phi$ satisfying the LSCC Property 2.5 of Section 2 .

To start with, let us record that curves of maximal slope (w.r.t. the relaxed slope $\left|\partial^{-} \phi\right|$ of $\phi$ ) indeed solve equation (8.12). This is ensured by the following result, which is a slight adaptation of [RSS11, Prop. 6.1, 6.2] and [RS06, Sect. 3.2], cf. also [AGS08, Prop. 1.4.1].

Proposition 8.3. Assume Property 2.5. There holds

$$
\left\|\partial_{\ell}^{\circ} \phi\right\|_{*}(u) \leq \inf \left\{\|\xi\|_{*}: \xi \in \partial_{\ell} \phi(u)\right\} \leq\left|\partial^{-} \phi\right|(u) \quad \text { for all } u \in D\left(\left|\partial^{-} \phi\right|\right) .
$$

Furthermore, if $\phi$ fulfills the chain rule w.r.t. $\partial_{\ell} \phi$, namely:

$$
\begin{gathered}
u \in H^{1}(0, T, \mathcal{B}), \xi \in L^{2}\left([0, T], \mathcal{B}^{*}\right), \xi \in \partial_{\ell} \phi(u) \text { a.e. in }(0, T) \\
\Rightarrow \phi \circ u \in A C([0, T]), \frac{\mathrm{d}}{\mathrm{d} t}(\phi \circ u)=\left\langle\xi, u^{\prime}\right\rangle \text { a.e. in }(0, T),
\end{gathered}
$$

then

(1) the relaxed slope $\left|\partial^{-} \phi\right|$ is a strong upper gradient for $\phi$;

(2) every curve of maximal slope $u$ w.r.t. $\left|\partial^{-} \phi\right|$ is a solution of the gradient flow (8.12) and fulfills the minimal section principle

$$
-\partial_{\ell}^{\circ} \phi(u(t)) \subset J\left(u^{\prime}(t)\right), \quad\left\|\partial_{\ell}^{\circ} \phi\right\|_{*}(u(t))=\left|\partial^{-} \phi\right|(u(t)) \quad \text { for a.a. } t \in(0, T) .
$$


This proposition paves the way to applying Theorem 3.6 and we have the following.

Corollary 8.4. Let $\phi: \mathcal{B} \rightarrow(-\infty, \infty]$ comply with the LSCC Property 2.5 and, in addition, with the condition of Proposition 8.3. Then, WED minimizers converge up to subsequences to a solution $u \in H^{1}(0, T ; \mathcal{B})$ of $(8.14)$.

Let us now present a classe of functionals to which Corollary 8.4 applies.

Dominated concave perturbations of convex functionals in Hilbert spaces. Let us now focus on the case in which $\mathcal{B}$ is a Hilbert space. In this context, a class of energies that complies with the hypotheses of Proposition 8.3 is given by functionals $\phi: D(\phi) \rightarrow(-\infty, \infty], D(\phi) \subset \mathcal{B}$, admitting the decomposition

$$
\begin{aligned}
& \phi=\psi_{1}-\psi_{2} \text { in } D(\phi), \\
& \psi_{1}: D(\phi) \rightarrow \mathbb{R} \text { convex and l.s.c., } \\
& \psi_{2}: D(\phi) \rightarrow \mathbb{R} \text { convex and l.s.c. in } D(\phi), D\left(\partial \psi_{1}\right) \subset D\left(\partial \psi_{2}\right) .
\end{aligned}
$$

It has been shown in [RS06, Theorem 4] that, if, in addition, $-\psi_{2}$ is a dominated concave perturbation of $\psi_{1}$, namely

$$
\begin{gathered}
\forall M \geq 0 \quad \exists \rho<1, \gamma \geq 0 \quad \text { such that } \forall u \in D\left(\partial \psi_{1}\right) \text { with } \max \{\phi(u),\|u\|\} \leq M \\
\sup _{\xi_{2} \in \partial \psi_{2}(u)}\left\|\xi_{2}\right\| \leq \rho\left\|\partial^{\circ} \psi_{1}(u)\right\|+\gamma,
\end{gathered}
$$

then $\phi$ satisfies the chain rule (8.13) with respect to the limiting subdifferential $\partial_{\ell} \phi$. Consequently, Proposition 8.3 applies.

If, in addition, $\phi$ complies with the LSCC properties, then Corollary 8.4 ensures that WED minimizers converge to a solution of the nonconvex gradient flow (8.14). We refer to [RS06] for further examples.

8.2. A class of gradient flows in Wasserstein spaces. Gradient flows in metric spaces classically arise as variational formulations of nonlinear parabolic PDEs is the space of probability measures. By referring to [AGS08] for all details, we consider here the nonlocal drift-diffusion equation

$$
\partial_{t} \rho-\operatorname{div}\left(\rho \nabla V+\frac{\nabla L_{F}(\rho)}{\rho}+\nabla W * \rho\right)=0 \quad \text { in } \mathbb{R}^{d} \times(0, \infty)
$$

where $V: \mathbb{R}^{d} \rightarrow \mathbb{R}$ acts as confinement potential, $L_{F}$ is defined as $L_{F}(r)=r F^{\prime}(r)-F(r)$ where $F:[0, \infty) \rightarrow \mathbb{R}$ is the internal-energy density, $W: \mathbb{R}^{d} \times \mathbb{R}^{d} \rightarrow \mathbb{R}$ is the interaction potential, and the symbol $*$ stands for classical convolution in $\mathbb{R}^{d}$.

Equation (8.17) can be formulated as a gradient flow in

$$
X=\mathscr{P}_{2}\left(\mathbb{R}^{d}\right)=\left\{\mu \in \mathscr{P}\left(\mathbb{R}^{d}\right): \int_{\mathbb{R}^{d}}|x|^{2} \mathrm{~d} \mu(x)<\infty\right\}
$$

endowed with the Wasserstein metric $\mathrm{d}=W_{2}$. The latter is classically given via

$$
W_{2}\left(\mu_{1}, \mu_{2}\right)=\min _{\gamma \in \Gamma\left(\mu_{1}, \mu_{2}\right)} \int_{\mathbb{R}^{d} \times \mathbb{R}^{d}}|x-y|^{2} \mathrm{~d} \gamma(x, y)
$$

where $\Gamma\left(\mu_{1}, \mu_{2}\right)=\left\{\gamma \in \mathscr{P}\left(\mathbb{R}^{d} \times \mathbb{R}^{d}\right): \pi_{\#}^{i} \gamma=\mu_{i}, i=1,2\right\}$ and $\pi_{\#}^{i}$ stands for the push-forward of the measure through the projection on the $i$-th component.

Let us consider the functional $\phi: \mathscr{P}_{2}\left(\mathbb{R}^{d}\right) \rightarrow(-\infty, \infty]$ given by the sum of the potential, the internal, and the interaction energy, namely

$$
\phi(\mu)= \begin{cases}\int_{\mathbb{R}^{d}} V(x) \mathrm{d} \mu(x)+\int_{\mathbb{R}^{d}} F(\rho(x)) \mathrm{d} x+\frac{1}{2} \int_{\mathbb{R}^{d} \times \mathbb{R}^{d}} W(x, y) \mathrm{d}(\mu \otimes \mu)(x, t) & \text { if } \mathrm{d} \mu=\rho \mathrm{d} x \\ \text { else }\end{cases}
$$


(i.e., $\phi(\mu)=\infty$ is $\mu$ is not absolutely continuous w.r.t. the Lebesgue measure). Define $\sigma$ to be the topology of narrow convergence in $\mathscr{P}_{2}\left(\mathbb{R}^{d}\right)$, namely $\mu_{n} \stackrel{\sigma}{\rightarrow} \mu$ iff

$$
\int_{\mathbb{R}^{d}} f(x) \mathrm{d} \mu_{n}(x) \rightarrow \int_{\mathbb{R}^{d}} f(x) \mathrm{d} \mu(x) \quad \forall f \in C_{b}\left(\mathbb{R}^{d}\right) \text { (continuous and bounded). }
$$

While referring to [AGS08] for a full discussion on this setting, we assume here that

$$
\begin{aligned}
& V: \mathbb{R}^{d} \rightarrow \mathbb{R} \text { is } \lambda \text {-convex, } \lim _{|x| \rightarrow \infty}|x|^{-2} V(x)=\infty, \\
& F:[0, \infty) \rightarrow \mathbb{R} \text { is convex and differentiable with } F(0)=0, \\
& \lim _{r \rightarrow \infty} F(r) / r=\infty, \quad F(r) / r^{\alpha} \text { is bounded below as } r \rightarrow 0+\text { for some } \alpha>d /(d+2), \\
& \exists C_{F} \geq 0 \forall x, y \geq 0: F(x+y) \leq C_{F}(1+F(x)+F(y)), \\
& r \mapsto r^{d} F\left(r^{-d}\right) \text { is convex and nonincreasing, } \\
& W: \mathbb{R}^{d} \times \mathbb{R}^{d} \rightarrow \mathbb{R} \text { is even, } \lambda \text {-convex,differentiable, and } \\
& \exists C_{W} \geq 0 \forall x, y \in \mathbb{R}^{d}: W(x+y) \leq C_{W}(1+W(x)+W(y))
\end{aligned}
$$

for some $\lambda \leq 0$. Let us record that these assumptions include the two classical choices $F(r)=r \ln r$ and $F(r)=r^{m}$ for $m>1$. By combining [AGS08, Thm. 11.1.3] and [RSS11, Prop. 7.4] one finds that

(1) the functional $\phi$ is $\lambda$-convex in $\mathscr{P}_{2}\left(\mathbb{R}^{d}\right)$;

(2) equation (8.17) can be equivalently written as

$$
v_{t}+\partial_{\mathrm{W}} \phi\left(\mu_{t}\right) \ni 0 \text { for a.a. } t \in(0, \infty)
$$

where the Borel velocity field $v_{t}=v(\cdot, t): \mathbb{R}^{d} \rightarrow \mathbb{R}^{d}, t \in(0, \infty)$, is associated with $\mu_{t}=\mu(\cdot, t)$ by letting $v_{t} \in L^{2}\left(\mathbb{R}^{d}, \mu_{t}\right),\left|\mu_{t}^{\prime}\right|=\|v(\cdot, t)\|_{L^{2}\left(\mathbb{R}^{d}, \mu_{t}\right)}^{2}$ and requiring the continuity equation

$$
\partial_{t} \mu+\operatorname{div}(v \mu)=0
$$

to hold in the sense of distributions in $\mathbb{R}^{d} \times(0, \infty)$. The Wasserstein subdifferential $\partial_{\mathrm{W}} \phi(\mu)$ in relation (8.23) at a measure $\mu \in D(\phi)$ is given by

$-v \in \partial_{\mathrm{W}} \phi(\mu) \Leftrightarrow-v \in L^{2}\left(\mathbb{R}^{d}, \mu\right)$ and

$\phi(\nu)-\phi(\mu) \geq \int_{\mathbb{R}^{d} \times \mathbb{R}^{d}}(-v(x)) \cdot(y-x) \mathrm{d} \gamma_{o}(x, y)+\frac{\lambda}{2} W_{2}^{2}(\mu, \nu) \quad$ for every $\nu$ in $\mathscr{P}_{2}\left(\mathbb{R}^{d}\right)$

where $\gamma_{o} \in \Gamma(\mu, \nu)$ is the unique optimal plan attaining the minimum in (8.18).

(3) curves of maximal slope for $\phi$ from (8.19) solve (8.23) and are unique.

We are now in the position of presenting the application of our abstract Theorem 3.6 in this setting.

Corollary 8.5. Assume (8.20)-(8.22). Then, the functional $\phi$ from (8.19) fulfills the LSCC Property 2.5 and is $\lambda$-geodesically convex on $\mathscr{P}_{2}$. In particular, the local slope $|\partial \phi|$ coincides with its relaxation $\left|\partial^{-} \phi\right|$ and it is an upper gradient. Thus, minimizers of the WED functionals converge to curves of maximal slope for $\phi$ w.r.t. $|\partial \phi|$.

Notice that in the previous statement the whole family of WED minimizers converge and there is no need to extract subsequences: this fact depends on the uniqueness of curves of maximal slope for $\phi$. Without entering into the details of the proof of Corollary 8.5, let us mention here that the LSCC Property 2.5 and the geodesic convexity of $\phi$ have been checked in [AGS08]. In particular, the slope $|\partial \phi|$ is a ( $L^{\infty}$-moderated) upper gradient [AGS08, Cor. 2.4.10] and Theorem 3.6 applies. 


\section{Appendix A. A Finsler distance induced by the Energy}

In this section we will briefly introduce Finsler extended distances induced by a general function $\mathfrak{f}: X \rightarrow[1, \infty]$ via

Definition A.1. The Finsler extended distance $\mathrm{d}_{\mathfrak{f}}$ associated with $\mathfrak{f}$ is defined by

$$
\mathrm{d}_{\mathfrak{f}}\left(u_{0}, u_{1}\right):=\inf \left\{\int_{a}^{b} \mathfrak{f}(\vartheta(t))\left|\vartheta^{\prime}\right|(t) \mathrm{d} t: \vartheta \in \operatorname{AC}([a, b] ; X), \quad \vartheta(a)=u_{0}, \vartheta(b)=u_{1}\right\}
$$

where $\mathrm{d}_{\mathfrak{f}}\left(u_{0}, u_{1}\right)=\infty$ if there are no absolutely continuous curves connecting $u_{0}$ to $u_{1}$.

It is easy to check that $d_{\mathfrak{f}}$ is an extended distance (i.e. it satisfies all the usual axioms defining a distance but it may assume the value $\infty$ ), satisfying

$$
\mathrm{d}_{\mathfrak{f}}\left(u_{0}, u_{1}\right) \geq \mathrm{d}\left(u_{0}, u_{1}\right) \quad \text { for all } u_{0}, u_{1} \in X .
$$

By a linear change of variable, it is also possibile to fix $[a, b]=[0,1]$ in (A.1).

In the following we assume that

$$
\mathfrak{f}: X \rightarrow[1, \infty] \quad \text { satisfies assumptions (2.19a) and (2.19b). }
$$

Our main motivating examples are provided by the choices

$$
\mathfrak{f}(x):=(\phi(x) \vee 1)^{1 / 2}, \quad \mathfrak{f}(x):= \begin{cases}1 & \text { if } \phi(x) \leq c \\ \infty & \text { otherwise. }\end{cases}
$$

Indeed, working with the distance induced by the latter functional would be helpful to force WED minimizers with bounded energy. On the other hand, we will see that the Finsler distance

$$
\mathrm{d}_{\phi} \text { induced via }\left(\text { A.1) by } \mathfrak{f}=(\phi \vee 1)^{1 / 2}\right.
$$

can be thought of as a natural metric in the context of WED minimization, cf. Theorem A.6 ahead.

Lemma A.2. Let $\left(u_{n}, v_{n}\right)$ be a sequence $\sigma$-converging to $(u, v)$ and let $\vartheta_{n} \in \mathrm{AC}\left(\left[a_{n}, b_{n}\right] ; X\right)$ be a sequence of curves connecting $u_{n}$ to $v_{n}$ and satisfying

$$
\liminf _{n \rightarrow \infty} \int_{a_{n}}^{b_{n}} \mathfrak{f}\left(\vartheta_{n}\right)\left|\vartheta_{n}^{\prime}\right| \mathrm{d} t \leq F<\infty .
$$

Then there exists a curve $\vartheta \in \mathrm{AC}([a, b] ; X)$ connecting $u$ to $v$ and satisfying $\int_{a}^{b} \mathfrak{f}(\vartheta)\left|\vartheta^{\prime}\right| \mathrm{d} t \leq F$.

Proof. By the reparametrization technique stated in Lemma 2.2 it is not restrictive to suppose that

$$
a_{n}=0,\left|\vartheta_{n}^{\prime}\right| \equiv 1 \quad \mathscr{L}^{1} \text {-a.e. in }\left(0, b_{n}\right), \quad b_{n} \leq \int_{0}^{b_{n}} \mathfrak{f}\left(\vartheta_{n}\right) \mathrm{d} s \leq F .
$$

Applying the compactness Theorem 2.6 (with $\mathfrak{f}$ instead of $\phi$ ) and setting $b:=\liminf _{n \rightarrow \infty} b_{n}$, we find a suitable subsequence $k \mapsto n_{k}$ and a limit 1-Lipschitz curve $\vartheta$ defined in $[0, b]$ such that

$$
\begin{gathered}
\vartheta_{n_{k}}(s) \stackrel{\sigma}{\rightarrow} \vartheta(s) \quad \text { for every } s \in[0, b), \quad \vartheta_{n_{k}}\left(b_{n_{k}}\right)=v_{n_{k}} \stackrel{\sigma}{\rightarrow} \vartheta(b)=v, \\
\int_{0}^{b} \mathfrak{f}(\vartheta)\left|\vartheta^{\prime}\right| \mathrm{d} s \leq \int_{0}^{b} \mathfrak{f}(\vartheta) \mathrm{d} s \leq \liminf _{k \rightarrow \infty} \int_{0}^{b_{n_{k}}} \mathfrak{f}\left(\vartheta_{n_{k}}\right) \mathrm{d} s \leq F .
\end{gathered}
$$

With our next result we examine the relationships between the properties of absolute continuity w.r.t. $d$ and $d_{f}$, and the related metric derivatives. We hence use the notation $\left|u^{\prime}\right|_{d}$ for the metric derivative w.r.t. $d$, to distinguish it from the metric derivative w.r.t. $d_{f}$.

Proposition A.3. If (A.3) holds, for every $u_{0}, u_{1} \in X$ at finite $d_{\mathfrak{f}}$-distance the inf in (A.1) is attained, $\mathrm{d}_{\mathfrak{f}}$ is lower semicontinuous in $X \times X$ with respect to the product topology induced by $\sigma$, and $\left(X, \mathrm{~d}_{\mathfrak{f}}\right)$ is a complete extended metric space. Moreover,

$u \in \mathrm{AC}\left([a, b] ;\left(X, \mathrm{~d}_{\mathfrak{f}}\right)\right) \quad$ if and only if $\quad u \in \mathrm{AC}([a, b] ;(X, \mathrm{~d}))$ and $(\mathfrak{f} \circ u)\left|u^{\prime}\right|_{\mathrm{d}} \in L^{1}(a, b)$

with, in this case, $\quad\left|u^{\prime}\right|_{\mathrm{d}_{\mathfrak{f}}}(t)=\mathfrak{f}(u(t))\left|u^{\prime}\right|_{\mathrm{d}}(t) \quad$ for a.a.t $\in(0, T)$. 
Proof. The $\sigma$-lower semicontinuity of $\mathrm{d}_{\mathfrak{f}}$ and the existence of minimizers for (A.1) are immediate consequences of the previous Lemma A.2. Then, $d_{f}$ is lower semicontinuous also with respect to $\mathrm{d}$ : since $(X, \mathrm{~d})$ is complete and $\mathrm{d}_{\mathfrak{f}} \geq \mathrm{d}$ we obtain the completeness of $\left(X, \mathrm{~d}_{\mathfrak{f}}\right)$.

As for (A.8), clearly any absolutely continuous curve $u$ on $[a, b]$ with $(\mathfrak{f} \circ u)\left|u^{\prime}\right|_{\mathrm{d}} \in L^{1}(a, b)$ is absolutely continuous with respect to the distance $\mathrm{d}_{\mathfrak{f}}$. Conversely, for every $u \in \operatorname{AC}\left([a, b] ;\left(X, \mathrm{~d}_{\mathfrak{f}}\right)\right)$ there holds

$$
\left|u^{\prime}\right|_{\mathrm{d}_{\mathfrak{f}}}(t) \geq \mathfrak{f}(u(t))\left|u^{\prime}\right|_{\mathrm{d}}(t) \quad \text { for a.a. } t \in(a, b) .
$$

This can be shown by adapting the argument from the proof of [RMS08, Lemma 6.4]. From (A.9), we infer that $(\mathfrak{f} \circ u)\left|u^{\prime}\right|_{\mathrm{d}} \in L^{1}(a, b)$. The converse inequality of (A.9) can be trivially checked, and (A.8) ensues.

Corollary A.4. If $(X, \mathrm{~d}, \sigma)$ is a compatible metric-topological space in the sense of $(2.18)$ and $\mathfrak{f}$ complies with $(\mathrm{A} .3)$, then also $\left(X, \mathrm{~d}_{\mathfrak{f}}, \sigma\right)$ is a compatible metric-topological space.

We conclude this section with the following equivalent representation for $d_{\mathfrak{f}}$.

Lemma A.5. For every $u_{0}, u_{1} \in X$

$$
\mathrm{d}_{\mathfrak{f}}\left(u_{0}, u_{1}\right)=\inf \left\{\int_{s_{0}}^{s_{1}}\left(\frac{1}{2}\left|\vartheta^{\prime}\right|^{2}+\frac{1}{2} \mathfrak{f}^{2}(\vartheta)\right) \mathrm{d} s: \vartheta \in \mathrm{AC}^{2}\left(\left[s_{0}, s_{1}\right] ; X\right), \vartheta\left(s_{i}\right)=u_{i}\right\},
$$

and the infimum is attained whenever it is finite.

Proof. By the Cauchy inequality it is immediate to check the inequality $\leq$ in (A.10). In order to prove the converse inequality let us suppose that $d_{\mathfrak{f}}\left(u_{0}, u_{1}\right)<\infty$ and let us choose an optimal curve $\vartheta \in \mathrm{AC}([0,1] ;(X, \mathrm{~d}))$ connecting $u_{0}$ to $u_{1}$; replacing $\vartheta$ with the reparametrized curve $\vartheta_{f}$ provided by (2.15) from Lemma 2.2 we conclude.

Properties of the value function with respect to the Finsler distance induced by the energy. Our next result shows that the value function enjoys finer properties with respect to $d_{\phi}$ from (A.5). Namely, $V_{\varepsilon}$ is indeed continuous w.r.t. $\mathrm{d}_{\phi}$ under the sole LSCC Property 2.5, whereas we have been able to prove its continuity w.r.t. d, along sequences with bounded energy, only under the additional $\lambda$-convexity condition (6.1). Furthermore, $G_{\varepsilon}$ is a strong upper gradient, in the standard sense of (2.4), w.r.t. $\mathrm{d}_{\phi}$, while $G_{\varepsilon}$ is just an $L^{1}$-moderated upper gradient (cf. (2.5)) w.r.t. the distance d.

Theorem A.6. Assume the LSCC Property 2.5. Then,

(1) for all $\left(x_{n}\right)_{n}, x \in X$ we have that $\mathrm{d}_{\phi}\left(x_{n}, x\right) \rightarrow 0$ as $n \rightarrow \infty$ implies $\lim _{n \rightarrow \infty} V_{\varepsilon}\left(x_{n}\right)=$ $V_{\varepsilon}(x)$

(2) for every curve $u \in \mathrm{AC}\left([a, b] ;\left(X, \mathrm{~d}_{\phi}\right)\right)$ the map $t \mapsto V_{\varepsilon}(u(t))$ is absolutely continuous, and there holds

$$
\left|\frac{\mathrm{d}}{\mathrm{d} t} V_{\varepsilon}(u(t))\right| \leq G_{\varepsilon}(u(t))\left|u^{\prime}\right|(t) \quad \text { for a.a. } t \in(a, b) .
$$

Proof. $\triangleright(1)$ : In order to show the continuity of $V_{\varepsilon}$, we argue as in the proof of Lemma 6.1. Namely, for a given minimizer $u_{\varepsilon} \in \mathscr{C}_{\varepsilon}(x)$ for $\mathcal{I}_{\varepsilon}$ (which exists since $x \in \mathrm{D}(\phi)$ ), we exhibit a sequence $\left(u_{n}\right)_{n}$ with $u_{n} \in \mathscr{C}_{\varepsilon}\left(x_{n}\right)$ for every $n \in \mathbb{N}$, fulfilling (6.3): in fact, observe that $\liminf _{n \rightarrow \infty} V_{\varepsilon}\left(x_{n}\right) \geq V_{\varepsilon}(x)$ again follows from Lemma 4.1 as $\mathrm{d}_{\phi}$-convergence implies $\sigma$-convergence via (A.2). To construct $\left(u_{n}\right)_{n}$, for every $n \in \mathbb{N}$ we consider an optimal curve $\vartheta_{n} \in \mathrm{AC}([0,1] ; X)$ for $\mathrm{d}_{\phi}\left(x_{n}, x\right)$. We exploit (2.15) from Lemma 2.2 to reparametrize the curves $\left(\vartheta_{n}\right)_{n}$ to curves $\tilde{\vartheta}_{n}:\left[0, \tau_{n}\right] \rightarrow X$ such that

$$
\int_{0}^{\tau_{n}}\left|\tilde{\vartheta}_{n}^{\prime}\right|^{2}(s) \mathrm{d} s=\int_{0}^{\tau_{n}}\left(1 \vee \phi\left(\tilde{\vartheta}_{n}(s)\right)\right) \mathrm{d} s=\int_{0}^{1}\left|\vartheta_{n}^{\prime}\right|(t)\left(1 \vee \phi\left(\vartheta_{n}(t)\right)\right)^{1 / 2} \mathrm{~d} t=\mathrm{d}_{\phi}\left(\bar{u}_{n}, \bar{u}\right) \rightarrow 0 .
$$

Hence, we define $u_{n}:[0, \infty) \rightarrow X$ by

$$
u_{n}(t):= \begin{cases}\tilde{\vartheta}_{n}(t) & t \in\left[0, \tau_{n}\right] \\ u_{\varepsilon}(t) & t \in\left[\tau_{n}, \infty\right)\end{cases}
$$


so that

$$
\mathcal{I}_{\varepsilon}\left[u_{n}\right]=\int_{0}^{\tau_{n}} \ell_{\varepsilon}\left(t, \tilde{\vartheta}_{n}(t),\left|\tilde{\vartheta}_{n}^{\prime}\right|(t)\right) \mathrm{d} t+\int_{\tau_{n}}^{\infty} \ell_{\varepsilon}\left(t, u_{\varepsilon}(t),\left|u_{\varepsilon}^{\prime}\right|(t)\right) \mathrm{d} t=: I_{1}+I_{2}
$$

Now,

$$
\begin{aligned}
I_{1} & =\int_{0}^{\tau_{n}} e^{-t / \varepsilon}\left(\frac{1}{2}\left|\tilde{\vartheta}_{n}^{\prime}\right|^{2}(t)+\frac{1}{\varepsilon} \phi\left(\tilde{\vartheta}_{n}(t)\right)\right) \mathrm{d} t \\
& \leq C \int_{0}^{\tau_{n}}\left(\left|\tilde{\vartheta}_{n}^{\prime}\right|^{2}(t)+\left(1 \vee \phi\left(\tilde{\vartheta}_{n}(t)\right)\right)\right) \mathrm{d} t=C \mathrm{~d}_{\phi}\left(\bar{u}_{n}, \bar{u}\right) \rightarrow 0
\end{aligned}
$$

as $n \rightarrow \infty$. On the other hand, we clearly have that $I_{2}$ converges to $\mathcal{I}_{\varepsilon}\left[u_{\varepsilon}\right]$ as $n \rightarrow \infty$ as $\tau_{n} \downarrow 0$. All in all, we have shown that $\lim \sup _{n \rightarrow \infty} \mathcal{I}_{\varepsilon}\left[u_{n}\right] \leq \mathcal{I}_{\varepsilon}\left[u_{\varepsilon}\right]$ as desired.

$\triangleright(2)$ : Let $u \in \operatorname{AC}\left([a, b] ;\left(X, \mathrm{~d}_{\phi}\right)\right)$ : then, $1 \vee(\phi \circ u) \in L^{1}(a, b)$. We again resort to (2.15) and reparametrize $u$ to a curve $\tilde{u}:[\tilde{a}, \tilde{b}] \rightarrow X$ fulfilling (A.12), so that $\tilde{u} \in \mathrm{AC}^{2}([\tilde{a}, \tilde{b}] ; X)$ and $\phi \circ \tilde{u} \in$ $L^{1}(\tilde{a}, \tilde{b})$. Therefore, $V_{\varepsilon} \circ \tilde{u} \in L^{1}(\tilde{a}, \tilde{b})$ and $G_{\varepsilon} \circ \tilde{u} \in L^{2}(\tilde{a}, \tilde{b})$, so that we are in a position to apply Thm. 4.6 and conclude that $s \mapsto V_{\varepsilon}(\tilde{u}(s))$ is absolutely continuous, with

$$
\left|\frac{\mathrm{d}}{\mathrm{d} s} V_{\varepsilon}(\tilde{u}(s))\right| \leq G_{\varepsilon}(\tilde{u}(s))\left|\tilde{u}^{\prime}\right|(s) \quad \text { for a.a. } s \in(\tilde{a}, \tilde{b}),
$$

which gives (A.11). This concludes the proof of (2).

\section{REFERENCES}

[AM17] G. Akagi, S. Melchionna, Elliptic-regularization of nonpotential perturbations of doubly-nonlinear gradient flows of nonconvex energies: A variational approach, J. Convex Anal. (2017), to appear.

[AMS17] G. Akagi, S. Melchionna, U. Stefanelli, Weighted Energy-Dissipation approach to doubly-nonlinear problems on the half line, J. Evol. Equ. (2017), to appear.

[AS10] G. Akagi, U. Stefanelli, A variational principle for doubly nonlinear evolution, Appl. Math. Lett. 23 (2010), 1120-1124.

[AS11] Weighted energy-dissipation functionals for doubly nonlinear evolution, J. Funct. Anal. 260 (2011), no. 9, 2541-2578. MR 2772344 (2012a:35019)

[AS14] Doubly nonlinear equations as convex minimization, SIAM J. Math. Anal. 46 (2014), no. 3, 1922-1945. MR 3215061

[AS16] A variational principle for gradient flows of nonconvex energies, J. Convex Anal. 23 (2016), $53-75$.

[Amb95] L. Ambrosio, Minimizing movements, Rend. Accad. Naz. Sci. XL Mem. Mat. Appl. (5) 19 (1995), 191-246. MR 97c:49044

[AF14] L. Ambrosio and J. Feng, On a class of first order Hamilton-Jacobi equations in metric spaces, J. Differential Equations 256 (2014), no. 7, 2194-2245. MR 3160441

[AGS08] L. Ambrosio, N. Gigli, and G. Savaré, Gradient flows in metric spaces and in the space of probability measures, second ed., Lectures in Mathematics ETH Zürich, Birkhäuser Verlag, Basel, 2008. MR MR2401600

[AT00] L. Ambrosio and P. Tilli, Selected topics on "analysis in metric spaces", Scuola Normale Superiore, Pisa, 2000.

[BCD97] M. Bardi and I. Capuzzo-Dolcetta, Optimal control and viscosity solutions of Hamilton-JacobiBellman equations, Systems \& Control: Foundations \& Applications, Birkhäuser Boston, Inc., Boston, MA, 1997, With appendices by Maurizio Falcone and Pierpaolo Soravia. MR 1484411 (99e:49001)

[BDM14] V. Bögelein, F. Duzaar, and P. Marcellini, Existence of evolutionary variational solutions via the calculus of variations, J. Differential Equations 256 (2014), no. 12, 3912-3942. MR 3190487

[BDM15] _ A time dependent variational approach to image restoration, SIAM J. Imaging Sci. 8 (2015), no. 2, 968-1006. MR 3341130

[BDMS15] V. Bögelein, F. Duzaar, P. Marcellini, and S. Signoriello, Nonlocal diffusion equations, J. Math. Anal. Appl. 432 (2015), no. 12, 398-428.

[BDMS17] _ Parabolic equations and the bounded slope condition, Ann. I. H. Poincaré AN 34 (2017), 355-379.

[Bré71] H. Brézis, Monotonicity methods in Hilbert spaces and some applications to nonlinear partial differential equations, Contribution to Nonlinear Functional Analysis, Proc. Sympos. Math. Res. Center, Univ. Wisconsin, Madison, 1971, Academic Press, New York, 1971, pp. 101-156.

[Bré73] H. Brézis, Opérateurs maximaux monotones et semi-groupes de contractions dans les espaces de Hilbert, North-Holland Publishing Co., Amsterdam, 1973, North-Holland Mathematics Studies, No. 5. Notas de Matemática (50). MR 50 \#1060 
[BE76] H. Brezis and I. Ekeland, Un principe variationnel associé à certaines équations paraboliques. Le cas indépendant du temps, C. R. Acad. Sci. Paris Sér. A-B, 282 (1976), no. 17, Aii, A971-A974, 1976.

[BE76b] H. Brézis and I. Ekeland, Un principe variationnel associé à certaines équations paraboliques. Le cas dépendant du temps, C. R. Acad. Sci. Paris Sér. A-B 282 (1976), no. 20, Ai, A1197-A1198. MR 0637215 (58 \#30593)

[Che99] J. Cheeger, Differentiability of Lipschitz functions on metric measure spaces, Geom. Funct. Anal. 9 (1999), no. 3, 428-517. MR 2000g:53043

[CO08] S. Conti, M. Ortiz, Minimum principles for the trajectories of systems governed by rate problems, J. Mech. Phys. Solids, 56 (2008), 1885-1904.

[CL71] M. G. Crandall and T. M. Liggett, Generation of semi-groups of nonlinear transformations on general Banach spaces, Amer. J. Math. 93 (1971), 265-298.

[CP69] M. G. Crandall and A. Pazy, Semi-groups of nonlinear contractions and dissipative sets, J. Functional Analysis 3 (1969), 376-418.

[DG93] E. De Giorgi, New problems on minimizing movements, Boundary Value Problems for PDE and Applications (Claudio Baiocchi and Jacques Louis Lions, eds.), Masson, 1993, pp. 81-98.

[DG96] E. De Giorgi, Conjectures concerning some evolution problems, Duke Math. J. 81 (1996), no. 2, 255-268, A celebration of John F. Nash, Jr. MR 1395405 (97f:35093)

[DGMT80a] E. De Giorgi, A. Marino, and M. Tosques, Problems of evolution in metric spaces and maximal decreasing curve, Atti Accad. Naz. Lincei Rend. Cl. Sci. Fis. Mat. Natur. (8) 68 (1980), no. 3, 180187. MR 83m:49052

[DGMT80b] E. De Giorgi, A. Marino, and M. Tosques, Problems of evolution in metric spaces and maximal decreasing curve, Atti Accad. Naz. Lincei Rend. Cl. Sci. Fis. Mat. Natur. (8) 68 (1980), no. 3, 180187. MR $83 \mathrm{~m}: 49052$

[Eva98] L. C. Evans. Partial differential equations, volume 19 of Graduate Studies in Mathematics. American Mathematical Society, Providence, RI, 1998.

[GS15] E. Gangbo and A. Swiech, Metric viscosity solutions of hamilton-jacobi equations depending on local slopes, Calc. Var. Partial Differential Equations, 54 (2015), no. 1, 1183-1218.

[GH96] M. Giaquinta and S. Hildebrandt, Calculus of variations i, Grundlehren der mathematischen Wissenschaften, vol. 310, Springer, Berlin, 1996.

[Gho09] N. Ghoussoub, Self-dual partial differential systems and their variational principles, Springer Monographs in Mathematics, Springer, New York, 2009. MR 2458698 (2010c:35001)

[GHN15] Y. Giga, N. Hamamuki, and A. Nakayasu, Eikonal equations in metric spaces, Trans. Amer. Math. Soc. 367 (2015), no. 1, 49-66. MR 3271253

[HK98] J. Heinonen and P. Koskela, Quasiconformal maps in metric spaces with controlled geometry, Acta Math. 181 (1998), no. 1, 1-61. MR 99j:30025

[Hir94] N. Hirano, Existence of periodic solutions for nonlinear evolution equations in Hilbert spaces, Proc. Amer. Math. Soc. 120 (1994), 185-192.

[Ilm94] T. Ilmanen, Elliptic regularization and partial regularity for motion by mean curvature, Mem. Amer. Math. Soc. 108 (1994), no. 520, x+90.

[JKO98] R. Jordan, D. Kinderlehrer, and F. Otto, The variational formulation of the Fokker-Planck equation, SIAM J. Math. Anal. 29 (1998), no. 1, 1-17 (electronic). MR 2000b:35258

[Kōm67] Y. Kōmura, Nonlinear semi-groups in Hilbert space, J. Math. Soc. Japan 19 (1967), 493-507.

[KN65] J. J. Kohn, L. Nirenberg, Non-coercive boundary value problems, Comm. Pure Appl. Math. 18 (1965), 443-492.

[LOR09] C. J. Larsen, M. Ortiz, C. L. Richardson, Fracture paths from front kinetics: relaxation and rate independence, Arch. Ration. Mech. Anal. 193 (2009), 539-583.

[LM17] M. Liero, S. Melchionna, The weighted energy-dissipation principle and evolutionary Gammaconvergence for doubly nonlinear problems, Submitted (2017).

[LS13a] M. Liero and U. Stefanelli, A new minimum principle for Lagrangian mechanics, J. Nonlinear Sci. 23 (2013), no. 2, 179-204. MR 3041623

[LS13b] Weighted inertia-dissipation-energy functionals for semilinear equations, Boll. Unione Mat. Ital. (9) 6 (2013), no. 1, 1-27. MR 3077111

[Lio63] J. L. Lions, Equations différentielles opérationelles dans les espaces de Hilbert, Centro Internazionale Matematico Estivo: Equazioni differenziali astratte, n. 2, Rome, Cremonese, 1963.

[Lio63b] J. L. Lions, Singular perturbations and some non linear boundary value problems, MRC Technical Summary Report 421, University of Wisconsin, 1963.

[Lio65] J. L. Lions, Sur certaines équations paraboliques non linéaires, (French) Bull. Soc. Math. France, 93 (1965), 155-175.

[Lio69] J.-L. Lions, Quelques méthodes de résolution des problèmes aux limites non linéaires, Dunod, Gauthier-Villars, Paris, 1969.

[LM72] J.L. Lions and E. Magenes, Non homogeneous boundary value problems and applications, vol. I, Springer, New York-Heidelberg, 1972. 
[MST89] A. Marino, C. Saccon, and M. Tosques, Curves of maximal slope and parabolic variational inequalities on nonconvex constraints, Ann. Scuola Norm. Sup. Pisa Cl. Sci. (4) 16 (1989), no. 2, 281-330. MR 91d:58035

[Mel16] S. Melchionna, A variational principle for nonpotential perturbations of gradient flows of nonconvex energies, J. Differential Equations, 262 (2016), 3737-3758.

[Mel17] S. Melchionna, A variational approach to symmetry, monotonicity, and comparison for doublynonlinear equations, J. Math. Anal. Appl. (2017), to appear.

[MO08] A. Mielke and M. Ortiz, A class of minimum principles for characterizing the trajectories and the relaxation of dissipative systems, ESAIM Control Optim. Calc. Var. 14 (2008), no. 3, 494-516. MR 2434063 (2010c:49024)

[MS11] A. Mielke and U. Stefanelli, Weighted energy-dissipation functionals for gradient flows, ESAIM Control Optim. Calc. Var. 17 (2011), no. 1, 52-85. MR 2775186 (2012c:35005)

[Mor06] B. Mordukhovich, Variational analysis and generalized differentiation. I, Springer-Verlag, Berlin, 2006.

[Nay76] B. Nayroles, Deux théorèmes de minimum pour certains systèmes dissipatifs, C. R. Acad. Sci. Paris Sér. A-B 282 (1976), no. 17, Aiv, A1035-A1038. MR 0418609 (54 \#6647)

[Ole64] O. A. Oleĭnik, On a problem of G. Fichera (Russian), Dokl. Akad. Nauk SSSR, 157 (1964), 1297-1300.

[Ott01] F. Otto, The geometry of dissipative evolution equations: the porous medium equation, Comm. Partial Differential Equations 26 (2001), no. 1-2, 101-174. MR 2002j:35180

[RMS08] R. Rossi, A. Mielke, and G. Savaré, A metric approach to a class of doubly nonlinear evolution equations and applications, Ann. Sc. Norm. Super. Pisa Cl. Sci. (5) 7 (2008), 97-169.

[RS03] R. Rossi and G. Savaré, Tightness, integral equicontinuity and compactness for evolution problems in Banach spaces, Ann. Sc. Norm. Super. Pisa Cl. Sci. (5) 2 (2003), no. 2, 395-431. MR 2005609

[RS06] Gradient flows of non convex functionals in Hilbert spaces and applications, ESAIM Control Optim. Calc. Var. 12 (2006), no. 3, 564-614 (electronic). MR MR2224826

[RSSS11] R. Rossi, G. Savaré, A. Segatti, and U. Stefanelli, A variational principle for gradient flows in metric spaces, C. R. Math. Acad. Sci. Paris, 349 (2011), 1224-1228.

[RSS11] R. Rossi, A. Segatti, and U. Stefanelli, Global attractors for gradient flows in metric spaces, J. Math. Pures Appl. (9) 95 (2011), no. 2, 205-244. MR 2763076

[ST12] E. Serra and P. Tilli, Nonlinear wave equations as limits of convex minimization problems: proof of a conjecture by De Giorgi, Ann. of Math. (2) 175 (2012), no. 3, 1551-1574. MR 2912711

[Seg13] A. Segatti, A variational approach to gradient flows in metric spaces, Boll. Unione Mat. Ital. (9), 6 (2013), no. 3, 765-780.

[ST16] E. Serra, P. Tilli, A minimization approach to hyperbolic Cauchy problems, J. Eur. Math. Soc. (JEMS), 18 (2016), 2019-2044.

[SS11] E. N. Spadaro, U. Stefanelli, A variational view at mean curvature evolution for linear growth functionals, J. Evol. Equ. 11 (2011), 793-809.

[Ste08] U. Stefanelli, The Brezis-Ekeland principle for doubly nonlinear equations, SIAM Journal on Control and Optimization 47 (2008), 1615.

[Ste11] The De Giorgi conjecture on elliptic regularization, Math. Models Methods Appl. Sci. 21 (2011), no. 6, 1377-1394. MR 2819200 (2012e:49024)

[TT17] L. Tentarelli, P. Tilli, De Giorgi's approach to hyperbolic Cauchy problems: the case of nonhomogeneous equations, Preprint (2017), arXiv:1709.09111.

[Vil09] C. Villani, Optimal transport. old and new, Grundlehren der Mathematischen Wissenschaften, vol. 338, Springer-Verlag, Berlin, 2009. MR MR2459454

[Vis08] A. Visintin, Extension of the Brezis-Ekeland-Nayroles principle to monotone operators, Adv. Math. Sci. Appl. 18 (2008), no. 2, 633-650. MR 2489147 (2010e:35298)

[Vis11] _ Structural stability of doubly-nonlinear flows, Boll. Unione Mat. Ital. (9) 4 (2011), no. 3, 363-391. MR 2906767 (2012k:35246)

[Vis13] _ Variational formulation and structural stability of monotone equations, Calc. Var. Partial Differential Equations 47 (2013), no. 1-2, 273-317. MR 3044140

[Vis15] W Weak structural stability of pseudo-monotone equations, Discrete Contin. Dyn. Syst. Ser. A, 35 (2015), 2763-2796. 
DIMI, Universitì di Brescia, Via Branze 38, I-25100 Brescia, Italy

E-mail address: riccarda.rossi@unibs.it

$U R L:$ http://riccarda-rossi.unibs.it

Dipartimento di Matematica, Università di Pavia, via Ferrata 1, I-27100 Pavia, Italy

E-mail address: giuseppe.savare@unipv.it

$U R L:$ http://www.imati.cnr.it/savare/

Dipartimento di Matematica "F. Casorati", via Ferrata 1, I-27100 Pavia, Italy

E-mail address: antonio.segatti@unipv.it

$U R L:$ http://www-dimat.unipv.it/segatti/ita/res.php

Faculty of Mathematics, University of Vienna, Oskar-Morgenstern-Platz 1, A-1090 Vienna, Austria and Istituto di Matematica Applicata e Tecnologie Informatiche E. Magenes - CNR, via Ferrata 1, I27100 PaVia, Italy

E-mail address: ulisse.stefanelli@univie.ac.at

$U R L:$ http://www.mate.univie.ac.at/ stefanelli 